\title{
A Novel Ka-Band Coupler with Wide Bandwidth and Excellent Phase Balance
}

\author{
By \\ Dylan Groven, B.Eng. \\ A thesis submitted to the Faculty of Graduate Studies and \\ Research in partial fulfillment of the requirement for the \\ degree of Master of Applied Science
}
Ottawa-Carleton Institute for Electrical and Computer Engineering Department of Electronics
Carleton University
Ottawa, Ontario, K1S 5B6
Canada

(C) Copyright December 2004, Dylan Groven 


$\begin{array}{ll}\begin{array}{l}\text { Library and } \\ \text { Archives Canada }\end{array} & \begin{array}{l}\text { Bibliothèque et } \\ \text { Archives Canada }\end{array} \\ \begin{array}{l}\text { Published Heritage } \\ \text { Branch }\end{array} & \begin{array}{l}\text { Direction du } \\ \text { Patrimoine de l'édition }\end{array} \\ \begin{array}{l}\text { 395 Wellington Street } \\ \text { Ottawa ON K1A ON4 } \\ \text { Canada }\end{array} & \begin{array}{l}\text { 395, rue Wellington } \\ \text { Ottawa ON K1A ON4 } \\ \text { Canada }\end{array}\end{array}$

Your file Votre référence ISBN: 0-494-00743-5

Our file Notre référence

ISBN: 0-494-00743-5

NOTICE:

The author has granted a nonexclusive license allowing Library and Archives Canada to reproduce, publish, archive, preserve, conserve, communicate to the public by telecommunication or on the Internet, loan, distribute and sell theses worldwide, for commercial or noncommercial purposes, in microform, paper, electronic and/or any other formats.

The author retains copyright ownership and moral rights in this thesis. Neither the thesis nor substantial extracts from it may be printed or otherwise reproduced without the author's permission.
AVIS:

L'auteur a accordé une licence non exclusive permettant à la Bibliothèque et Archives Canada de reproduire, publier, archiver, sauvegarder, conserver, transmettre au public par télécommunication ou par l'Internet, prêter, distribuer et vendre des thèses partout dans le monde, à des fins commerciales ou autres, sur support microforme, papier, électronique et/ou autres formats.

L'auteur conserve la propriété du droit d'auteur et des droits moraux qui protège cette thèse. $\mathrm{Ni}$ la thèse ni des extraits substantiels de celle-ci ne doivent être imprimés ou autrement reproduits sans son autorisation.
In compliance with the Canadian

Privacy Act some supporting forms may have been removed from this thesis.

While these forms may be included in the document page count, their removal does not represent any loss of content from the thesis.
Conformément à la loi canadienne sur la protection de la vie privée, quelques formulaires secondaires ont été enlevés de cette thèse.

Bien que ces formulaires aient inclus dans la pagination, il n'y aura aucun contenu manquant. 


\begin{abstract}
This thesis details the theory and design of a novel Ka-band coupler with unique broadband properties. The coupler consists of a CPW-slotline T-junction with a CPWlotline transition on either side of the T. One of the transitions is flipped so that a $180^{\circ}$ phase shift is created at the output. The phase balance is maintained over a $31.5 \mathrm{GHz}$ bandwidth, from $445 \mathrm{MHz}$ to $32 \mathrm{GHz}$. The useful bandwidth is limited to $10 \mathrm{GHz}$ due to return loss. The coupler was simulated using Momentum ${ }^{\mathrm{TM}}$ and Ensemble ${ }^{\mathrm{TM}}$, and was fabricated using Phillips $^{\mathrm{TM}}$, OMMIC ${ }^{\mathrm{TM}}$ ED02AH process.

A single-balanced mixer was designed with an RF frequency of $35 \mathrm{GHz}$ and an IF frequency of $5 \mathrm{GHz}$ using a unique topology to demonstrate one application for the novel coupler. The mixer was simulated using $\operatorname{ADS}^{\mathrm{TM}}$ and fabricated. Experimental data and conclusions are given for both the novel coupler and single-balanced mixer.
\end{abstract}




\section{Acknowledgements}

It has been my pleasure to work at two great research institutions while pursuing graduate studies - Carleton University and the Communications Research Centre (CRC). Special thanks are due to Jim Wight, my academic supervisor, for allowing me to pursue a master's degree in the first place, while also serving as an inspiration in my chosen field. Special thanks are also due my two research advisors at CRC, Dr. Malcolm Stubbs and Dr. Khelifa Hettak, who are leaders in their field of research and from whom I received a great deal of guidance. Other people at CRC I'd like to thank by name, who helped me at various stages of research for this thesis, are Kees Verver and Carole Glaser. Finally, warm thanks to some of the professors I encountered through my studies: Dr. Langis Roy, Dr. Calvin Plett, both at Carleton University, and Dr. Derek McNamara from the University of Ottawa. 


\section{TABLE OF CONTENTS}

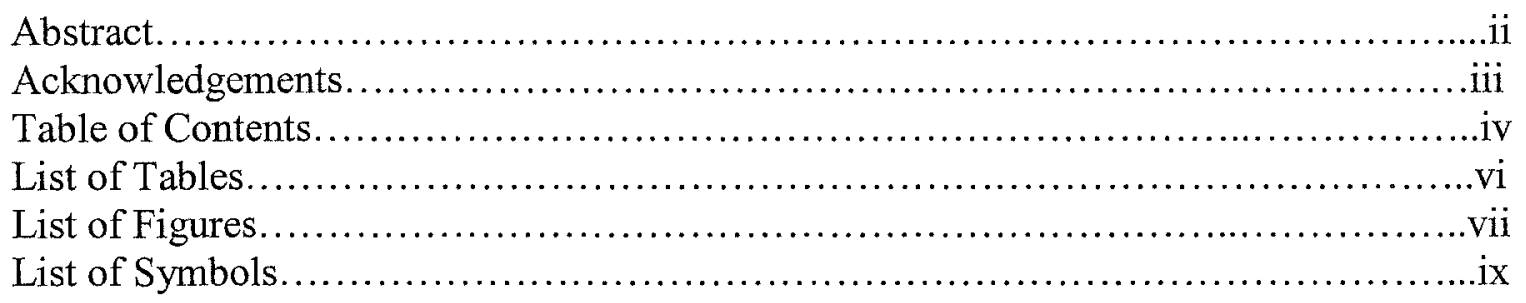

CHAPTER 1: INTRODUCTION..................................................

1.1 THE NEED FOR BROADBAND COMMUNICATIONS $\ldots \ldots \ldots \ldots \ldots \ldots \ldots \ldots \ldots . . \ldots \ldots$

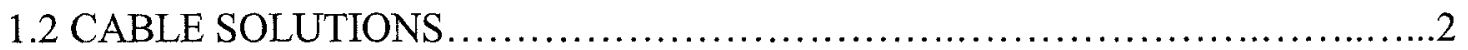

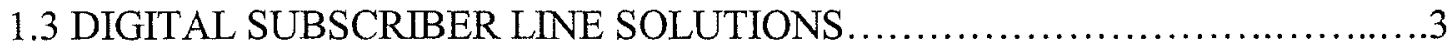

1.4 LMDS AND OTHER FIXED WIRELESS SOLUTIONS $\ldots \ldots \ldots \ldots \ldots \ldots \ldots \ldots . .5$

1.5 THESIS OBJECTIVES...................................................

CHAPTER 2: DESIGN BACKGROUND........................................

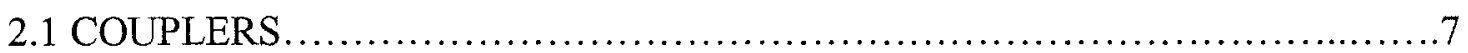

2.1.1 Branch-Line Coupler................................................... 8

2.1.2 Hybrid-Ring Hybrid ................................................... 10

2.1.3 Coupled-Line Coupler.................................................... 12

2.2 VARIOUS TRANSMISSION LINES ....................................... 14

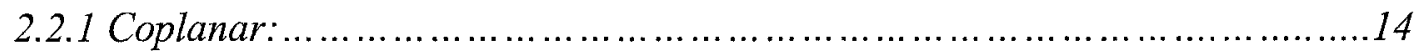

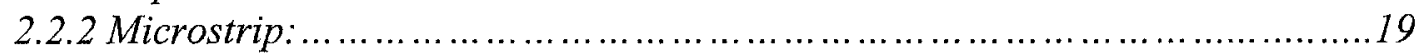

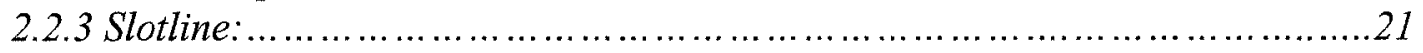

2.3 COMPARISON BETWEEN CPW, MICROSTRIP, AND SLOTLINE ..........23

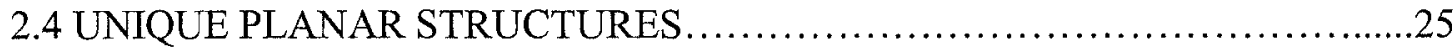

CHAPTER 3: DESIGN OF PLANAR COUPLER...................................29

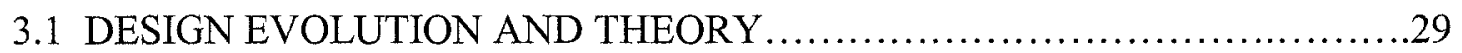

3.2 TRANSITION AND COUPLER DESIGNS .................................40

3.2 .1 Transition \#1 design: ............................................................ 41

3.2 .2 Coupler \#1 design: ............................................................ 43

3.2 .3 Transition $\# 2$ design: ................................................................ 44

3.2 .4 Coupler \#2 design: ............................................................... 45

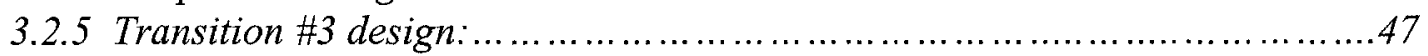

3.2 .6 Coupler \#3 design: ............................................................ 49

3.2 .7 Transition \#4 design: ....................................................... 50

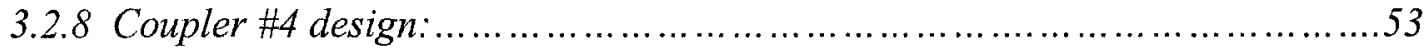

3.3 FINAL TRANSITION AND COUPLER DESIGN .......................... 55

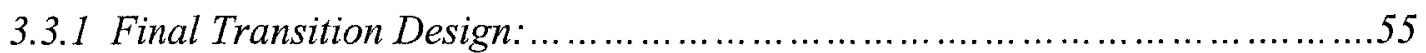

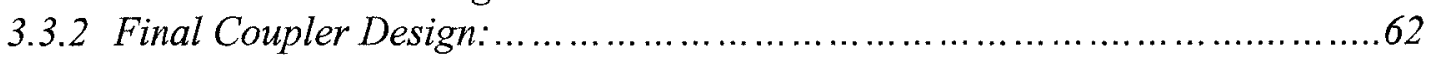


CHAPTER 4: NEW COUPLER USED IN BASIC MIXER.......................66

4.1 MIXER TOPOLOGIES USING VARIOUS TYPES OF

COUPLERS.........................................................66

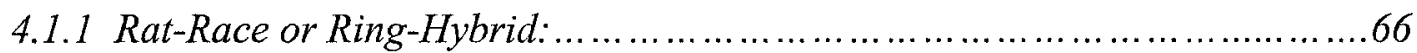

4.1.2 Branchline coupler:......................................................68

4.1.3 Marchand Balun and Parallel-Line Baluns:................................70

4.1.4 Mixer Topology and Layout using new coupler.............................. 71

4.2 DETAILS OF MIXER DESIGN USING THE NEW COUPLER ................. 75

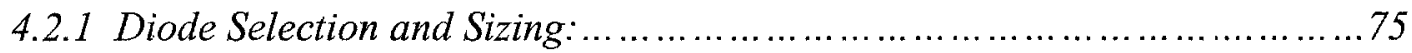

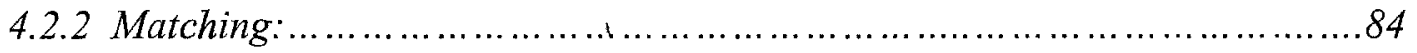

4.2 .3 Filters:......................................................................... 91

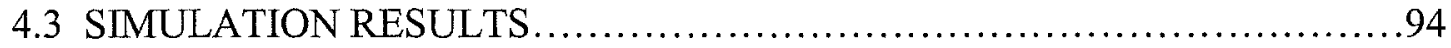

CHAPTER 5: MEASURED RESULTS.........................................98

5.1 MEASURED COUPLER RESULTS ........................................ 98

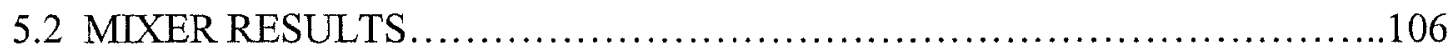

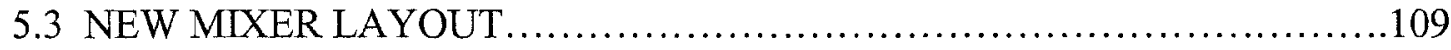

CHAPTER 6: CONCLUSIONS AND FUTURE WORK...........................114

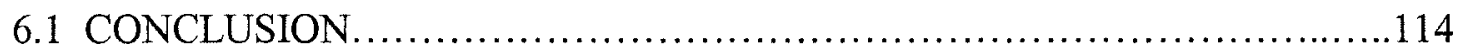

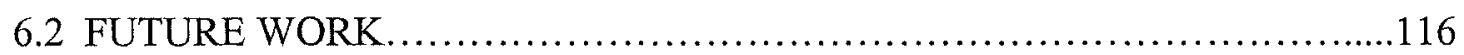

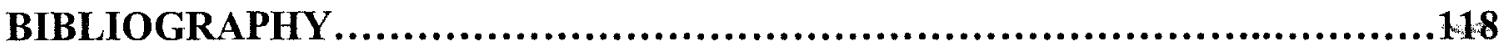

APPENDIX A: EVEN-ODD MODE ANALYSIS OF VARIOUS COUPLERS....121

A.1 BRANCLINE COUPLER ............................................. 121

Matlab Program \#1: Branchline Coupler ....................................124

A.2 HYBRID-RING COUPLER:......................................... 125

Matlab Program \#2: Hybrid-Ring Coupler ..................................... 128

A.3 COUPLED-LINE COUPLER:.............................................. 130

Matlab Program \#3: Coupled-Line Coupler......................................131 


\section{List of Tables}

TABLE 2-1: SUMMARY OF PERFORMANCE OFIDEAL COUPLERS IN THE $20 \mathrm{GHZ}$ TO $40 \mathrm{GHZ}$

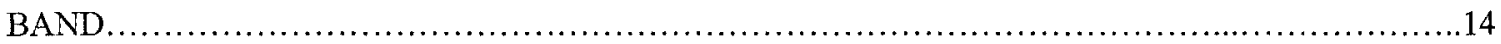

TABLE 2-2: LIMITS OF ZO FOR VARIOUS TRANSMISSION LINES $[16$, P.431] ...................24

TABLE 2-3: QUALITATIVE COMPARISON OF VARIOUS MIC LINES [16, P.434] .................25

TABLE 3-1: COMPARISON OF SIMULATED PERFORMANCE OF NEW COUPLER WITH IDEAL

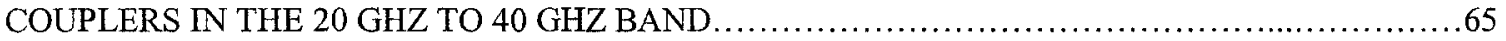

TABLE 4-1: REFLECTION COEFFICIENT AT THE LO, RF, AND IF PORTS ......................90

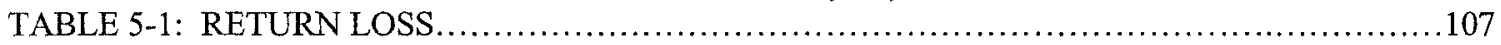

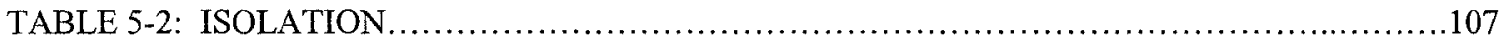

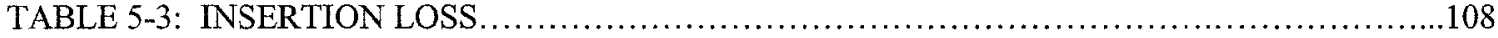

TABLE 5-4: REFLECTION COEFFICIENTS AT THE LO, RF, AND IF PORTS .....................112 


\section{List of Figures}

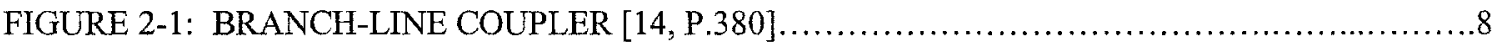
FIGURE 2-2: CHANGE OF AMPLITUDE AND PHASE OVER FREQUENCY OF AN IDEAL

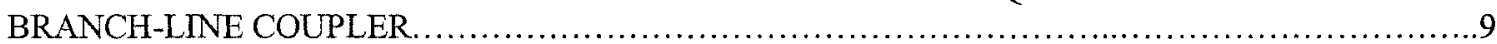
FIGURE 2-3: HYBRID-RING COUPLER [14, P.403] .............................................. FIGURE 2-4: CHANGE OF AMPLITUDE AND PHASE OVER FREQUENCY OF AN IDEAL

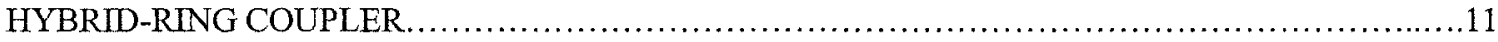
FIGURE 2-5: A) COUPLED-LINE COUPLER, WITH (B) SCHEMATIC CIRCUIT [14, P.389] .........12 FIGURE 2-6: CHANGE OF AMPLITUDE AND PHASE OVER FREQUENCY OF AN IDEAL COUPLED-LINE COUPLER.................................................................... FIGURE 2-7: CPW SHOWING (A) ASPECT RATIO A/B, AND (B) FIELD DISTRIBUTION FOR

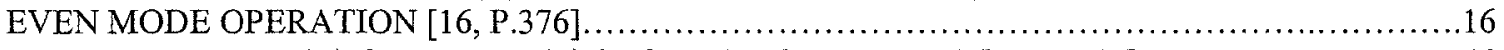
FIGURE 2-8: CPW (A) OPEN AND (B) SHORT CIRCUIT STUBS [16, P.443] ....................18 FIGURE 2-9: CPW (A) SHUNT AND (B) SERIES STUB CONFIGURATIONS [16, P.444]...........19 FIGURE 2-10: (A) MICROSTRIP LINE AND (B) FIELD DISTRIBUTION [14, P.161] ..................20 FIGURE 2-11: SLOT LINE SHOWING FIELD DISTRIBUTION [16, P.270] ........................21

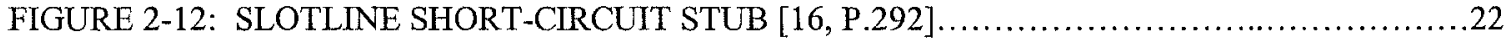
FIGURE 2-13: SLOTLINE OPEN-CIRCUIT STUB WITH (A) A FLARED OPEN END, (B) A CIRCULAR DISC OPEN END, AND (C) A COMBINATION OF FLARED SLOT AND HALF-DISC

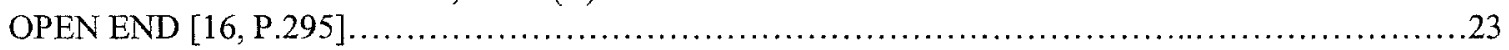

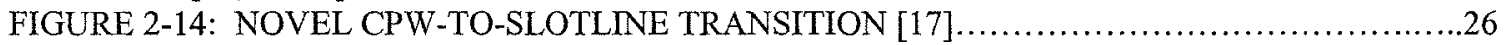

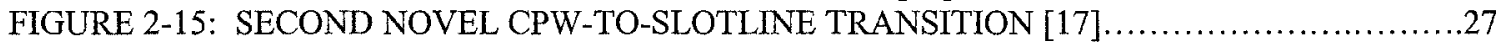
FIGURE 2-16: ELECTRIC FIELD DISTRIBUTION OF THREE TYPES OF SLOTLINE

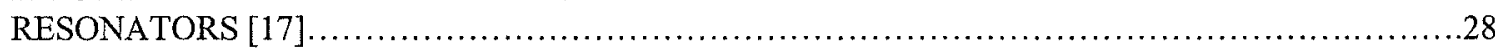

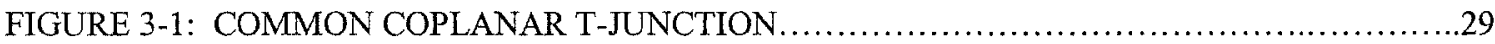
FIGURE 3-2: COPLANAR T-JUNCTION WITH 1800 PHASE SPLIT BETWEEN OUTPUT PORTS..30

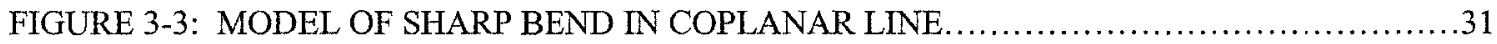
FIGURE 3-4: MODEL OF MODIFIED BEND FOR REDUCING PARASITIC REACTANCE ...........32 FIGURE 3-5: T-JUNCTION WITH DANGLING SLOTS ........................................ 33 FIGURE 3-6: T-JUNCTION WITH TERMINATED SLOTS ............................................ FIGURE 3-7: CPW-SLOTLINE TRANSITIONS WITH CPW-SLOTLINE T-JUNCTION.................34 FIGURE 3-8: (A) EQUIVALENT CIRCUIT AND (B) BLOCK DIAGRAM OF CPW-SLOTLINE

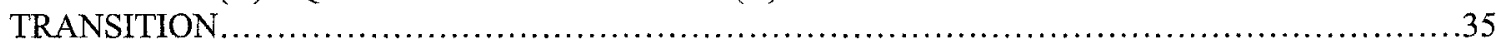
FIGURE 3-9: SERIES STUB ADDED TO THE EQUIVALENT CIRCUIT ........................... FIGURE 3-10: BLOCK DIAGRAM FOR THE TRANSITION WITH A SERIES STUB $\ldots \ldots \ldots \ldots \ldots . \ldots 37$

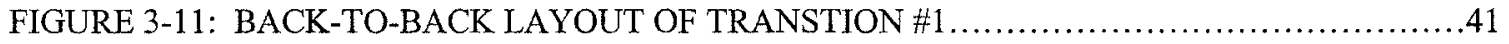

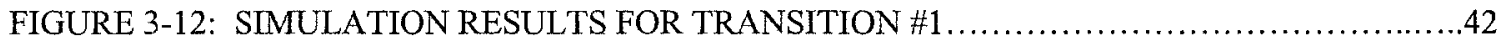

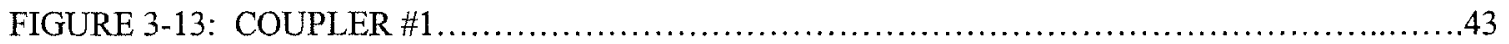

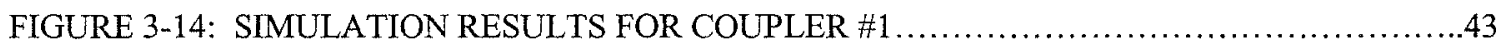

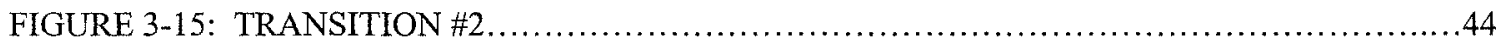

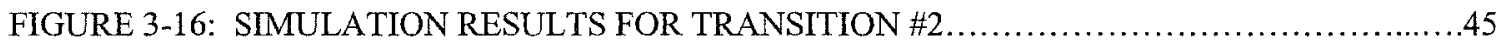

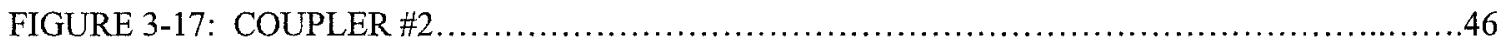

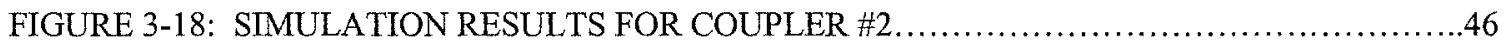

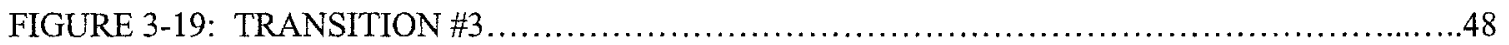

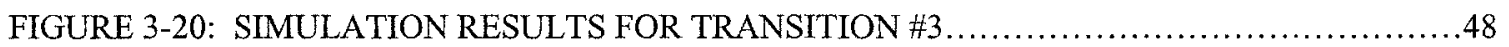

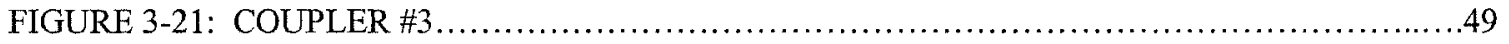

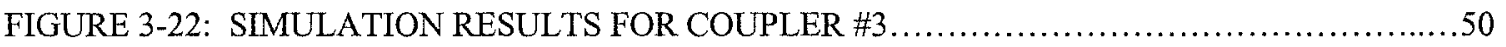
FIGURE 3-23: EQUIVALENT CIRCUIT OF TWO STUBS IN PARALLEL [2] ......................

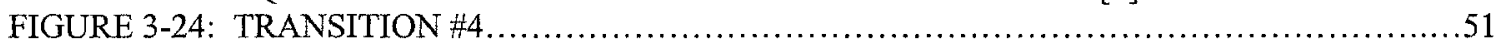

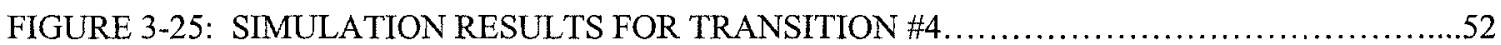

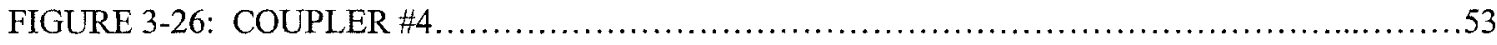

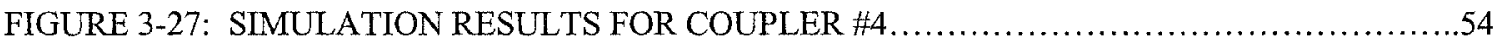




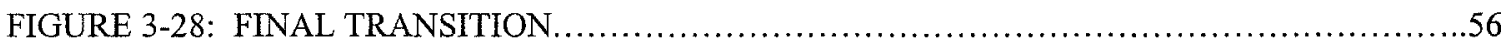

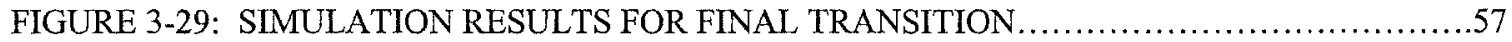

FIGURE 3-30: FINAL TRANSITION WITHOUT SERIES MICROSTRIP STUB - TRANSITION \#6...60

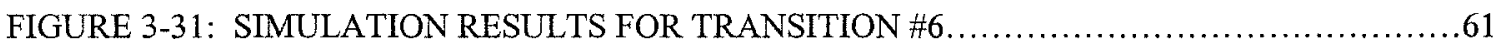

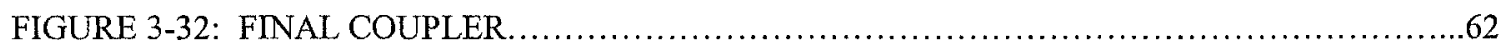

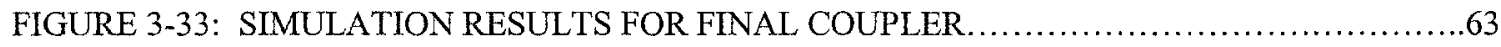

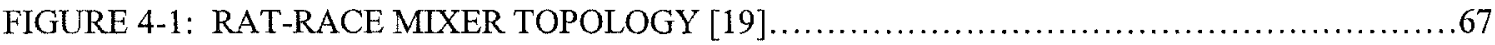

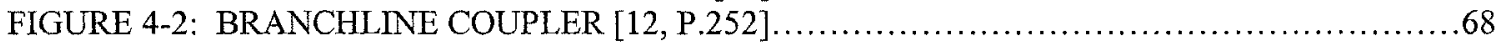

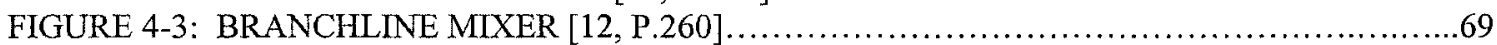

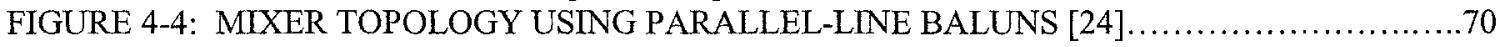

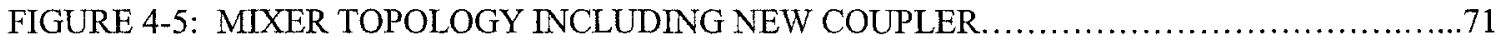

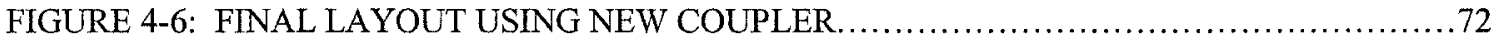

FIGURE 4-7: TEST CIRCUIT WITH NEW COUPLER AND DIODES FROM THE OMMICTM

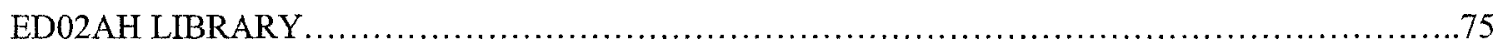

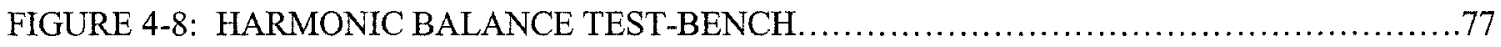

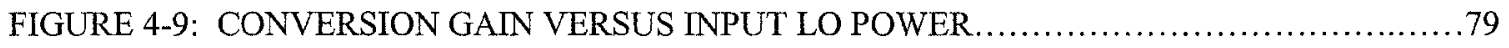

FIGURE 4-10: V-I CURVE FOR THE 'GM' DIODES USED IN THE MIXER TEST CIRCUIT ..........80

FIGURE 4-11: CONVERSION GAIN VERSUS RF INPUT POWER $\ldots \ldots \ldots \ldots \ldots \ldots \ldots \ldots \ldots \ldots \ldots . . . \ldots 1$

FIGURE 4-12: CONVERSION GAIN VERSUS FINGER LENGTH.................................... 82

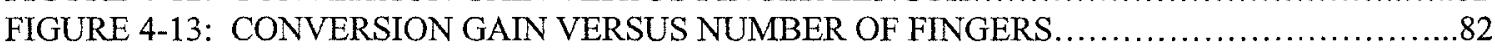

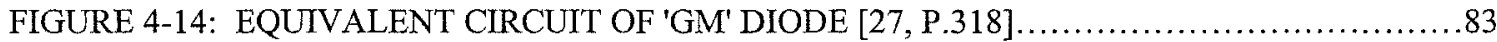

FIGURE 4-15: A) MATCHING CIRCUIT AT RF PORT, B) MATCHING CIRCUITS AT THE DIODES, C) MATCHING CIRCUIT AT THE IF PORT, AND D) MATCHING SHUNT CAPACITOR AT LO

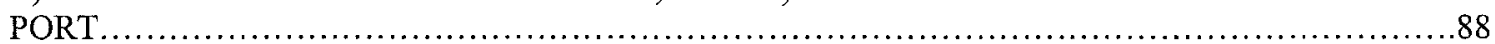

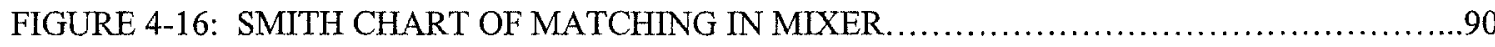

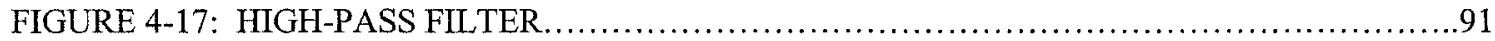

FIGURE 4-18: SIMULATED S-PARAMETER RESULTS FOR HIGH-PASS FILTER ...............92

FIGURE 4-19: LOW-PASS FILTER ............................................................... 93

FIGURE 4-20: SIMULATED S-PARAMETER RESULTS FOR LOW-PASS FILTER $\ldots \ldots \ldots \ldots \ldots \ldots \ldots . . . . . . . . . .93$

FIGURE 4-21: (A) CONVERSION GAIN AND (B) PORT-TO-PORT ISOLATION ...................94

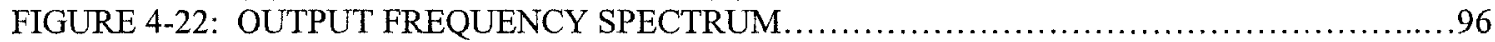

FIGURE 4-23: SIMULATED GAIN COMPRESSION FOR MIXER DESIGN $\ldots \ldots \ldots \ldots \ldots \ldots \ldots \ldots \ldots . . \ldots 7$

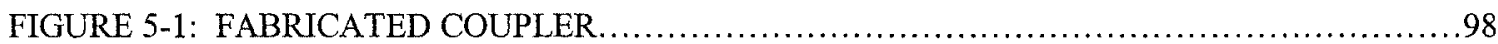

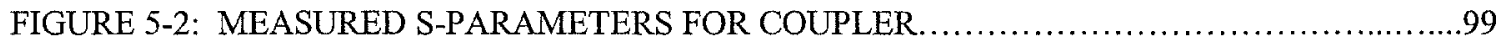

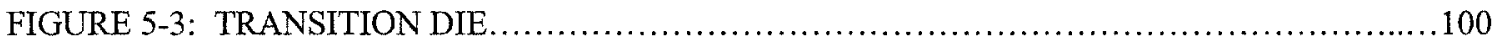

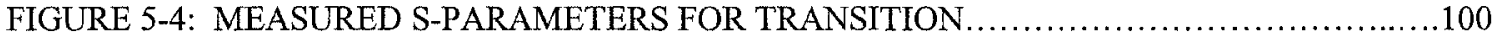

FIGURE 5-5: DATA FROM MOMENTUMTM, ENSEMBLETM, AND EMPIRETM SUPERIMPOSED

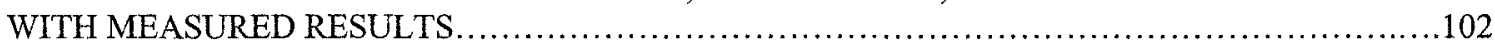

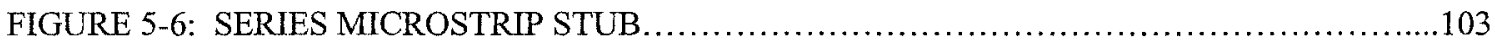

FIGURE 5-7: SIMULATION RESULTS FOR SERIES MICROSTRIP STUB $\ldots \ldots \ldots \ldots \ldots \ldots \ldots \ldots \ldots \ldots$

FIGURE 5-8: COUPLER MEASUREMENTS FOR AIR GAP WITH MOMENTUM SIMULATIONS

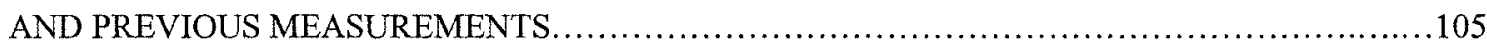

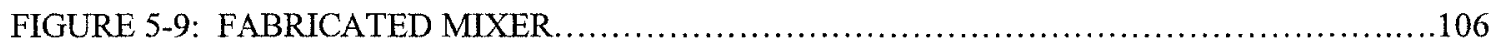

FIGURE 5-10: MIXER WITH ADDED QUARTER-WAVELENGTH SHUNT STUB

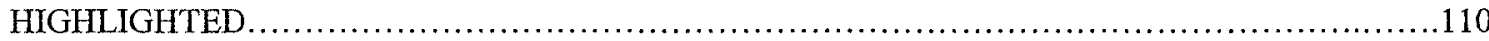

FIGURE 5-11: S-PARAMETERS FOR IF GROUND STUB ...................................111

FIGURE 5-12: (A) CONVERSION GAIN AND (B) PORT-TO-PORT ISOLATION FOR NEW MIXER

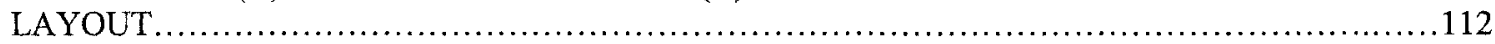




\section{List of Symbols}

\begin{tabular}{|c|c|}
\hline$Z_{\mathrm{CPW}}$ & Characteristic impedance of coplanar line \\
\hline$\varepsilon_{\text {eff }}$ & Effective dielectric constant \\
\hline$K\left(k_{1}\right), K\left(k_{1}^{\prime}\right)$ & Complete elliptic integrals \\
\hline$\varepsilon_{\mathrm{T}}$ & Relative dielectric constant \\
\hline$k_{0}$ & Field used in elliptic integral \\
\hline$k_{0}^{\prime}$ & Field used in elliptic integral \\
\hline$k_{1}$ & Field used in elliptic integral \\
\hline$k_{1}^{\prime}$ & Field used in elliptic integral \\
\hline$Z_{\text {OSLOT }}$ & Characteristic impedance of the slotline \\
\hline $\mathrm{Z}_{\mathrm{OCPW}}$ & Characteristic impedance of coplanar line \\
\hline$Z_{\text {SSlot }}$ & Characteristic impedance of shunt slotline stub \\
\hline$\phi$ & Electrical length of transmission line \\
\hline$Z_{\mathrm{SCPW}}$ & Characteristic impedance of the series stub \\
\hline $\mathrm{w} / \mathrm{d}$ & Aspect ratio for the microstrip line \\
\hline$\varepsilon_{\mathrm{e}}$ & Effective dielectric constant for microstrip line \\
\hline $\mathrm{Z}_{0}$ & Characteristic impedance for a parallel plate waveguide \\
\hline$\eta$ & Intrinsic impedance of the parallel plate waveguide \\
\hline $\mathrm{G}_{\mathrm{c}}$ & Conversion gain \\
\hline$\delta_{1}$ & Degradation at the RF frequency \\
\hline $\mathrm{R}_{\mathrm{S}}$ & Diode series resistance \\
\hline$r_{d r}$ & Nonlinear junction resistance at the $\underline{R F}$ frequency \\
\hline
\end{tabular}


Angular RF frequency

$\mathrm{C}_{\mathrm{j} 0}$

Variable capacitor in Figure 4-14

IL

Insertion loss

$\Gamma$

Reflection coefficient 


\section{CHAPTER 1: INTRODUCTION}

\subsection{The need for Broadband Communications}

One could say the digital revolution is in its second era, the first era being the invention and adoption of the personal computer, the second era represented by the creation of the Internet. Just as Gordon Moore, founder of Intel, predicted exponential growth in the speed of microprocessors, the same law seems to be revealing itself in the growth of communications networks. Just to put the growth in perspective, "when Bill Clinton was inaugurated as President of the United States there were 200 domain hosts in use. As of January 1, 2003 there were 171,000,000 domain hosts in use." [1] This number of hosts could represent anywhere between 17 billion and 30 billion individual web pages, but the truth is that nobody really knows. The exchange of all this information on the web is made even more challenging on the infrastructure by the introduction of multimedia. The exchange of pictures, music, movies, and streaming video is pushing the limits of old $56 \mathrm{k}$ modems, and driving demand for broadband service from cable and digital subscriber line (DSL) providers. Obvious bottlenecks are surfacing with this increase in demand for bandwidth, one of which is the final link to the subscriber termed "the last mile". The last mile is the communication link serviced currently by cable modems, DSL modems, Fiber to the curb solutions, and fixed wireless applications. Each of these solutions has strengths and weaknesses, some of which will be discussed in the next few paragraphs. 


\subsection{Cable Solutions}

Some facts about cable modems used to deliver broadband service: Worldwide cable modem service subscriptions will climb from 32.8 million for 2003 to 69.4 million in 2008 [3]. Assuming a price of $\$ 40$ a month per subscription, this represents a $\$ 33$ billion market (in U.S. dollars) for cable modems alone by 2008. One key advantage for cable broadband service providers is the ability to bundle services [2]. The United States accounted for $46.7 \%$ of worldwide cable modem subscribers in 2003 [3]. The U.S. is the only country where cable is leading DSL. Cable networks were originally built to distribute television signals, and therefore had a broadcast architecture. This means the network was designed to originally support only high-volume traffic downstream to the subscriber. This architecture evidently does not support internet traffic, which requires two-way communication. Furthermore, the original network consisted of long strings of amplifiers to amplify the traveling RF signals, however these strings of amplifiers introduced a lot of noise, reduced the bandwidth, and was prone to faults, since any one failed amplifier would bring the link down [2]. Cable networks were upgraded in the 80's and 90's with hybrid-fiber-coax (HFC) cables, which combined single-mode optical fiber with regular copper coax [2]. This eliminated the need for so many amplifiers on the trunk lines, improving reliability, as well as increasing the available bandwidth. It soon became apparent, with the growth of the internet, that the existing cable networks could be exploited by providing two-way communication. This is the foundation of today's broadband cable network internet service, which delivers $6 \mathrm{MHz}$ bandwidth per channel at frequencies of 54 to $550 \mathrm{MHz}$ downstream, and 5 to $42 \mathrm{MHz}$ upstream [2]. This translates to (depending on modulation schemes) 10 to $30 \mathrm{Mb} / \mathrm{s}$ downstream and 
$768 \mathrm{~kb} / \mathrm{s}$ to $10 \mathrm{Mb} / \mathrm{s}$ upstream [2]. The main disadvantage for cable networks is the cost. The copper coaxial cable used from the last network node to the subscriber can only extend so far, so the bulk of the network must be upgraded to HFC cables, which is a costly endeavor. Sources say that cable service providers spend as much as US $\$ 1000$ per customer on equipment and infrastructure [4]. This means that at $\$ 40$ a month on average per customer subscription, it takes 2 years of continuous subscription to turn a profit.

\subsection{Digital Subscriber Line Solutions}

Digital subscriber line (DSL) technology was first introduced in 1989 by Bellcore. It was designed to be a system that could send video and television signals on telephone networks, which could compete with cable television companies [5]. Essentially, DSL was created in order to maximize the use of existing telephone network infrastructure. There are 790 million copper loops in the world's telephone networks, and this infrastructure is the key asset for telephone companies [6]. It only makes economic sense that these companies want to find as many uses for their network as possible. The DSL circuit consists of a DSL modem on each end of a twisted-pair telephone line. There are three information channels provisioned for in the bandwidth of the line: a high-speed downstream channel, a medium-speed upstream channel, and a channel for regular phone service [5]. The speeds of transfer upstream and downstream are determined by which flavor of DSL one is subscribing to. There are actually 7 flavors to choose from: ADSL, CDSL, G.Lite, HDSL, RADSL, SDSL, and VDSL; each defined by varying bandwidths, or features such as symmetry in the upstream and downstream speeds [5]. VDSL has the 
fastest bit rate, at up to $26 \mathrm{Mbps}$ upstream and $50 \mathrm{Mbps}$ downstream. This is significantly higher than what can be provided by cable modems, however the one caveat is that VDSL cannot be deployed over a distance beyond roughly 300 meters [5].

The advantages of DSL are that it is cost effective, since the technology is just expanding the use of an existing network of copper cable, unlike with cable modems, where a large amount of capital must be invested in laying hybrid-fiber-coax cable [5]. The DSL chipset market is also mature, which means highly integrated DSL modems are available and cheap [5]. Another big advantage is that DSL is a dedicated service, whereas with cable modems the bandwidth is shared with other households on the same node. It has been an issue with cable Internet service in the past that the cable company would oversubscribe for its infrastructure, leading to less-than-posted bandwidth being available to each customer. The major disadvantage for DSL is the fact that it is limited to dense populations where the central office is close to the subscribers. Distance limitations of DSL are due to noise, loss, and interference on the copper line, especially in older homes. This makes DSL an ineffective technology for more rural or even suburban communities - a key disadvantage that will be highlighted in the next paragraphs.

\subsection{LMDS and other Fixed Wireless Solutions}

A novel solution to providing last mile coverage to the subscriber has also evolved from humble broadcast television technology in the last several years - fixed wireless. Fixed wireless is the space occupied by Local Multipoint Distribution Communication Systems (LMDS), Microwave Video Distribution Systems (MVDS), and Multi-channel 
Multipoint Distribution Systems (MMDS), all in the GHz bandwidth range [7]. This space is also being populated by the various flavors of the IEEE's 802.11 standard, which operates in the $2.4 \mathrm{GHz}$ and $5 \mathrm{GHz}$ unlicensed bands offering roughly $100 \mathrm{MHz}$ of bandwidth. In fact the market for LMDS products was flat for a number of years, but now the adoption of 802.11 networks that provide lower bandwidth is seen as a bridge technology that introduces customers to the benefits of wireless service, which will then fuel the adoption of higher bandwidth LMDS networks in the future. Up to this point in this discussion, the two key disadvantages posed by cable and DSL networks have been shown to be cost and limits of reach. Fixed wireless hubs can be deployed in spacings of $4 \mathrm{kms}$, covering $80 \%$ of households, and costing between $\$ 100$ and $\$ 200$ per subscriber in infrastructure costs [8]. This represents a savings of roughly $80 \%$ in up-front costs as compared with cable and DSL deployed infrastructure. As such, there is great potential for fixed wireless networks to be deployed as disruptive technology by newer players in the broadband market. To summarize the benefits of deploying fixed wireless networks, they offer lower infrastructure costs, lower operating costs, fast time to market, scalability, and can offer higher bit rate [8].

At this point, several solutions to bringing broadband connections to customers have been discussed, with fixed wireless systems presenting an attractive alternative. As just discussed, a key advantage of fixed wireless networks is the low cost. One of the drivers of cost in LMDS systems are the microwave integrated circuits needed. LMDS systems operate in the $28 \mathrm{GHz}$ band in North America, and the 38 or $40 \mathrm{GHz}$ band in Europe [9]. These frequencies typically require GaAs circuits, which are expensive. Given this fact, 
chip real estate on GaAs wafers is very valuable and it is necessary to develop techniques that reduce circuit sizes or techniques for maximizing substrate area use. Key components in these communication circuits are the couplers used in mixers, frequency multipliers, power amplifiers, modulators, and a number of other essential communication circuits. These couplers tend to be large and irregularly shaped, rendering large areas on the wafer useless. Many couplers are also quite narrowband, requiring the manufacture of different components for different frequency bands; it is useful and cost efficient in terms of manufacturing to design components that can be used across a wide bandwidth.

\subsection{Thesis objectives and outline}

The purpose of this thesis is to highlight some of the areas where current couplers used in microwave circuits are deficient, and explore the design of a new breed of coupler - one that is broadband, and that uses layout area more efficiently. The focus of the design of a new coupler is directed at LMDS systems, therefore the frequencies of interest are between $20 \mathrm{GHz}$ and $40 \mathrm{GHz}$, as this band contains the frequency bands used for LMDS systems. The ideal result would be to create a coupler that functions well at both $20 \mathrm{GHz}$ and $40 \mathrm{GHz}$, thus allowing the design to be used in either North American or European LMDS systems.

The chapters of this thesis are organized as follows: Chapter 2 provides background information leading up to the design of a novel coupler, including an overview of existing couplers, a discussion on different transmission lines available for design, and a discussion on some unique techniques for designing new structures developed at the 
Communications Research Centre. Chapter 3 provides the theory and design evolution of the novel coupler leading to a final design that was ultimately fabricated and tested. Chapter 4 details the design of a single-balanced mixer employing the novel coupler designed in chapter 3 . Chapter 5 provides measured results for the new coupler and the single-balanced mixer, as well as a discussion on the results and an improved design for the mixer. Finally, chapter 6 yields conclusions and an outline for future work. 


\section{CHAPTER 2: DESIGN BACKGROUND}

This chapter provides some background on common couplers to provide a basis for comparing the new coupler design, which will be discussed in later chapters. This chapter also provides an overview of the planar transmission lines, including basic theory for application, that were used in the new coupler design. Finally, some novel structures developed at the Communications Research Centre are described. Several of these structures were incorporated in the various design iterations of the new coupler.

\subsection{Couplers}

For comparison purposes in this thesis, the focus is directed to couplers for which all ports are unbalanced, which excludes the Marchand balun, double-Y balun, and derivatives thereof. There are four main types of couplers that fit this criteria. These are the branch-line coupler, the hybrid-ring coupler, the Lange coupler, and the coupled-line coupler. The following sections yield an exploration of the transfer functions of each of these couplers in order to reveal insights necessary to proceed with the design of the new coupler. 


\subsubsection{Branch-line Coupler}

A figure of a branch-line coupler is given in Figure 2-1 below.

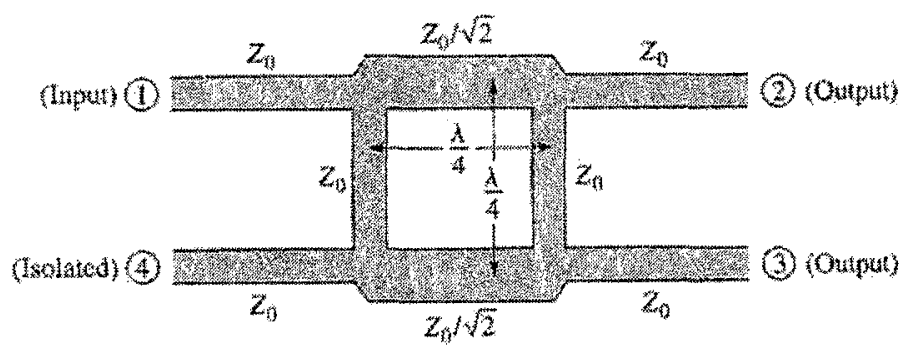

Figure 2-1: Branch-line coupler [10, p.380]

As illustrated in Figure 2-1, the branch-line coupler has four ports, each matched to the characteristic impedance $Z_{0}$. Output ports $2 \& 3$ are split in phase by $90^{\circ}$, making the branch-line a quadrature coupler. Output port 4 is isolated, meaning no power goes to it from port 1. The coupler consists of two quarter-wavelength branches with a characteristic impedance of $Z_{0}$, and two branches with a characteristic impedance of $Z_{0} / \sqrt{2}$. It is sometimes difficult to realize the impedances in the branches, and because of the need for quarter-wavelength lines, this circuit is suitable only for narrow bandwidth operations $[11$, p.252].

The branch-line coupler is a simple coupler to design and fabricate, and it has low loss. Because it does not require bond wires or narrow microstrip lines, it can be fabricated successfully on soft substrates to realize low-cost circuits. The branch-line hybrid has a relatively narrow bandwidth, approximately 10 percent, and the VSWRs and transmission bandwidths of different pairs of ports are not the same [12, p.217]. One way to derive the transfer function of the branch-line coupler is by applying Even-Odd mode theory. 
Detailed Even and Odd mode analysis of branch-line couplers for deriving the $S_{21}$ transfer function can be found in [10, p.380], and in Appendix A. A Matlab program was derived based on the transfer function for $\mathrm{S}_{21}$ for an ideal branch-line coupler and can also be found in Appendix A. A plot of changes in the amplitude and phase over frequency from $20 \mathrm{GHz}$ to $40 \mathrm{GHz}$ for the transfer function of an ideal branch-line coupler are given in Figure 2-2 below.

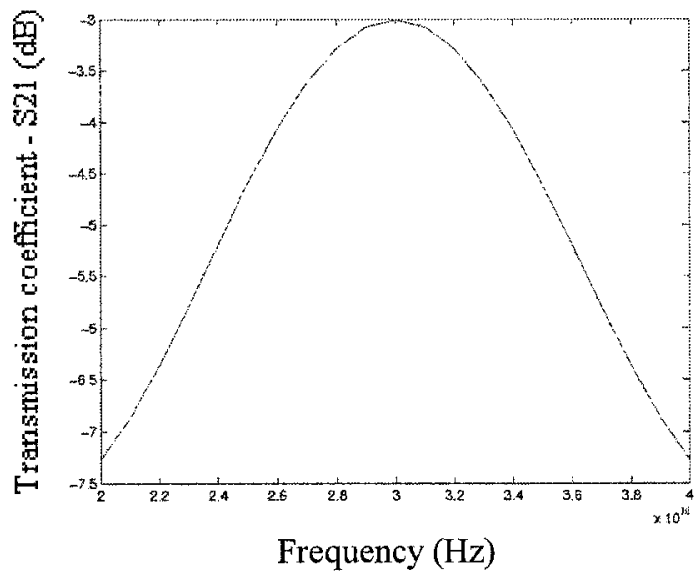

(a)

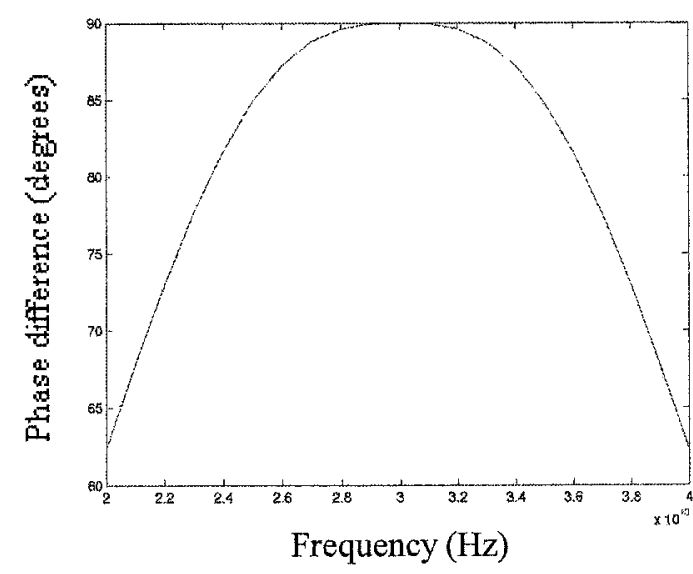

(b)

Figure 2-2: Change of amplitude and phase over frequency of an ideal branch-line coupler.

Figure 2-2 shows that both the amplitude and phase difference have limited bandwidths within the $20 \mathrm{GHz}$ to $40 \mathrm{GHz}$ band of interest. Figure 2-2(a) shows that the amplitude response of the ideal branch-line coupler has a $-1 \mathrm{~dB}$ bandwidth of $8 \mathrm{GHz}$. Figure 2-2(b) shows that the phase response of the branch-line coupler has a $-5^{\circ}$ bandwidth of $10 \mathrm{GHz}$. 


\subsubsection{Hybrid-Ring Hybrid}

A figure of a Hybrid-Ring coupler is given in Figure 2-3 below.

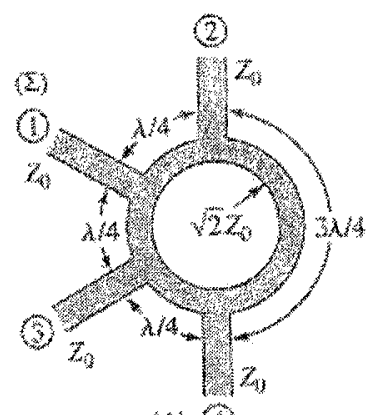

(a) 4

Figure 2-3: Hybrid-Ring coupler [10, p.403]

Figure 2-3 shows that the hybrid ring also has 4 ports. An input signal to port 1 is evenly split with no phase difference between ports 2 and 3 because these ports are equidistant from port 1 , while port 4 is isolated from port 1 because the distances in both directions around the ring from port 1 are such that the signals combine in opposite phases and thus cancel each other out. An input signal to port 4 is split evenly between ports 2 and 3 with a $180^{\circ}$ phase split, while port 1 is isolated. Therefore the Hybrid Ring coupler is the planar equivalent of a magic-T. Like the Branch-line coupler, the Hybrid-Ring coupler is very popular "because of its relatively low loss and the simplicity of its design and fabrication." The ring hybrid requires frequency-sensitive elements, namely transmission lines of a precise length that make it a narrow-band component [12, p.215]. "The bandwidth of this hybrid is approximately $10 \%$ to $15 \%$, although when the effects of junction discontinuities and transmission line dispersion are included, it may be slightly lower [11, p.244-246]". 
As was the case for the Branch-line coupler, the $S_{21}$ transfer function for an ideal HybridRing coupler was derived based on Even and Odd mode analysis in [10, p.405].

Appendix A contains the derivation of the transfer function, as well as a Matlab program for plotting the amplitude and phase response of the ideal Hybrid-Ring coupler. Figure 2-4 gives the graphs of the amplitude and phase response from $20 \mathrm{GHz}$ to $40 \mathrm{GHz}$.

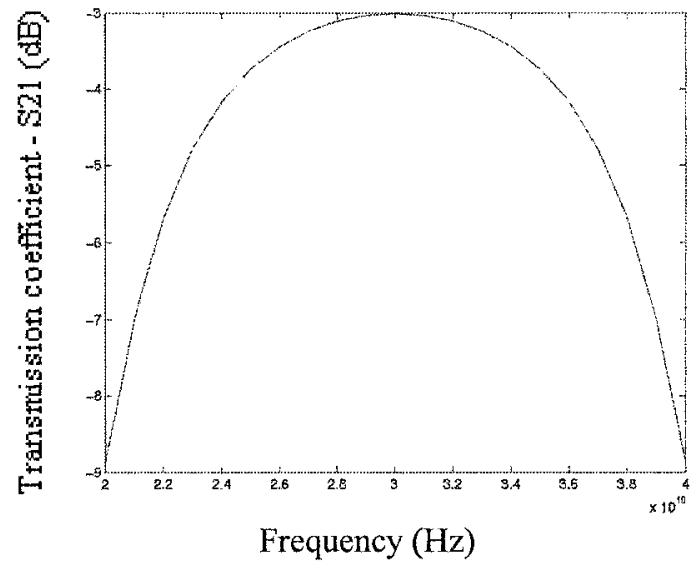

(a)

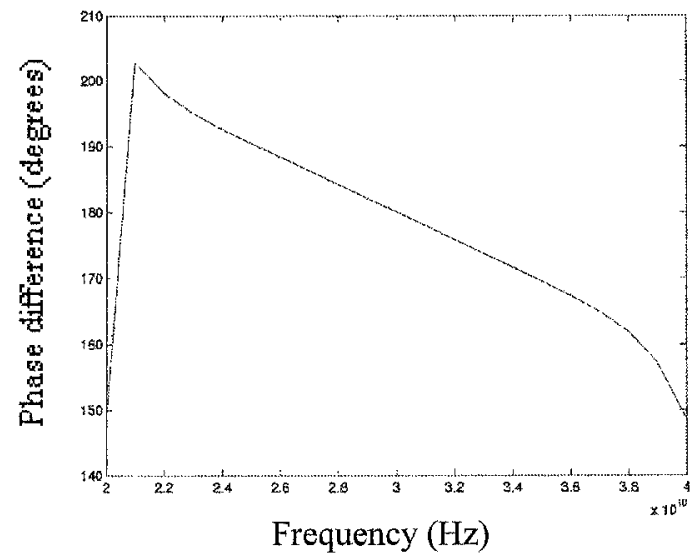

(b)

Figure 2-4: Change of amplitude and phase over frequency of an ideal hybrid-ring coupler.

Figure 2-4 (a) shows that the Hybrid-Ring coupler has a $-1 \mathrm{~dB}$ amplitude bandwidth of $12 \mathrm{GHz}$, which is greater than that for the Branch-line coupler. Figure 2-4(b) shows that the Hybrid-Ring coupler has a phase response $+/-5^{\circ}$ bandwidth of $5 \mathrm{GHz}$, which is lower than that found for the Branch-line coupler. The Hybrid-Ring is therefore a tradeoff of greater amplitude response bandwidth at the cost of some phase response balance, as compared to the Branch-line coupler. 


\subsubsection{Coupled-Line Coupler}

The Coupled-Line coupler and the Lange coupler are in the same family. The Lange coupler is essentially a Coupled-Line coupler that is divided into a number of sections to improve the coupling coefficient. Therefore, for the purpose of this thesis, it is only necessary analyze the basic Coupled-Line coupler for comparison with the previous couplers discussed. Figure 2-5 shows the Coupled-Line coupler.

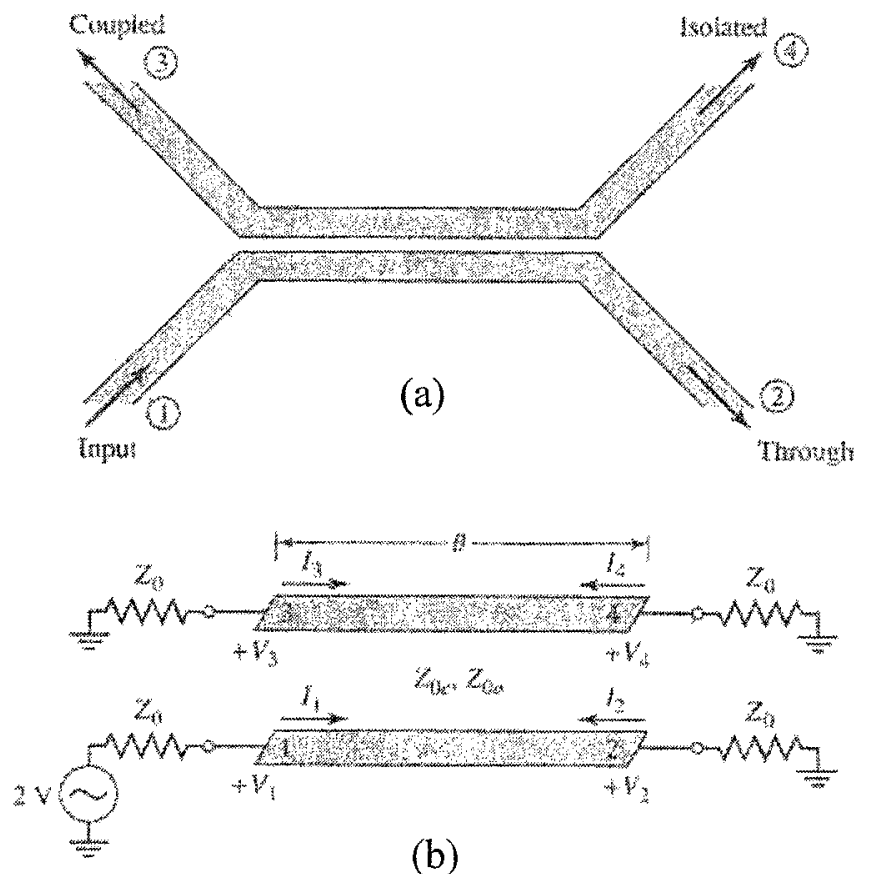

Figure 2-5: a) Coupled-Line coupler, with (b) schematic circuit [10, p.389]

Figure 2-5(a) shows that the Coupled-Line coupler is very simple to design. It consists mainly of two quarter-wavelength lengths of line in close proximity. A signal injected into port 1 is split between port 2 and 3 in amounts that depend on the coupling coefficient, $\mathrm{C}$, and the frequency. The signals at port 2 and 3 are phase split by $90^{\circ}$, 
making this another quadrature coupler. The advantage of the Coupled-Line coupler is that the output signals will always have a phase split of $90^{\circ}$. This is an inherent quality that is frequency independent. The disadvantage is that it is almost impossible to achieve the $-3 \mathrm{~dB}$ insertion loss limit, which is the theoretical maximum level of coupling [10, p.389].

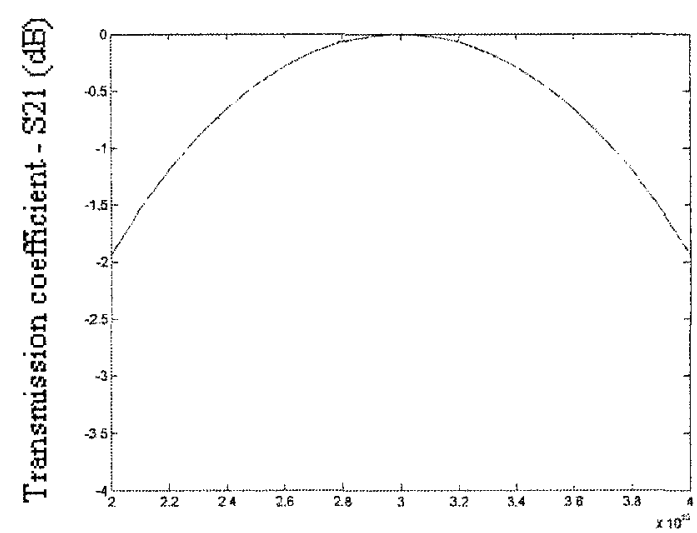

Frequency $(\mathrm{Hz})$

(a)

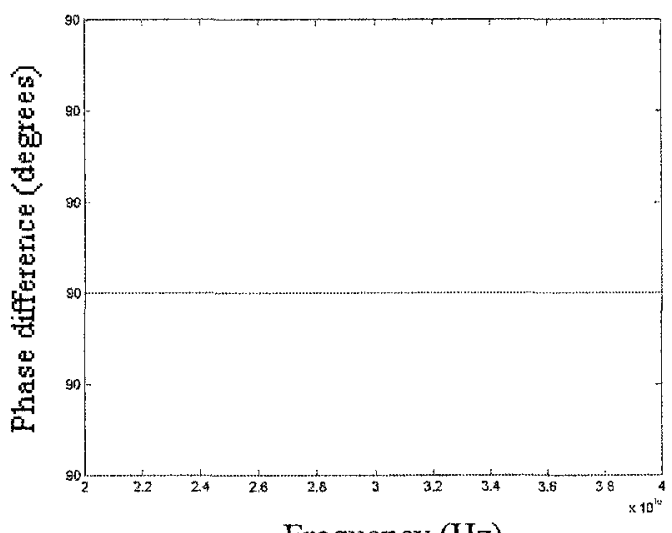

Frequency $(\mathrm{Hz})$

(b)

Figure 2-6: Change of amplitude and phase over frequency of an ideal Coupled-Line coupler.

As predicted, Figure 2-6(b) shows that the ideal Coupled-Line coupler maintains a $90^{\circ}$ phase split over the entire $20 \mathrm{GHz}$ to $40 \mathrm{GHz}$ band. The same is not true for the amplitude response in Figure 2-6(a), which has a bandwidth of $14 \mathrm{GHz}$. This is due to the fact that the dimensions, and consequently the $\mathrm{Z}_{0 \mathrm{e}}$ and $\mathrm{Z}_{0 \mathrm{o}}$ characteristic impedances, of the Coupled-Line coupler are frequency dependent making this structure relatively narrowband [10, p.389]. 
The following table summarizes the performance in the $20 \mathrm{GHz}$ to $40 \mathrm{GHz}$ band of the various ideal couplers discussed in this section.

Table 2-1: Summary of performance of ideal couplers in the $20 \mathrm{GHz}$ to $40 \mathrm{GHz}$ band.

\begin{tabular}{|c|c|c|}
\hline & $\begin{array}{l}\text { Amplitude } \\
\text { Response BW }\end{array}$ & $\begin{array}{c}\text { Phase } \\
\text { Response BW }\end{array}$ \\
\hline $\begin{array}{l}\text { Branchline } \\
\text { coupler }\end{array}$ & $8 \mathrm{GHz}$ & $10 \mathrm{GHz}$ \\
\hline $\begin{array}{l}\text { Mybrid-Ring } \\
\text { Coupler }\end{array}$ & $12 \mathrm{GHz}$ & $5 \mathrm{GHz}$ \\
\hline $\begin{array}{l}\text { Coupled Iine } \\
\text { Coupler }\end{array}$ & $14 \mathrm{GHz}$ & (Infinite) \\
\hline
\end{tabular}

\subsection{Various Transmission Lines}

In the design of a new coupler to be discussed in the coming chapters, a number of planar transmission lines and structures are employed. A brief overview of these transmission lines and structures is provided in the next sections.

\subsubsection{Coplanar:}

The coplanar waveguide (CPW) offers several advantages over conventional microstrip line. It simplifies fabrication. It facilitates easy shunt as well as series surface mounting of active and passive devices. It eliminates the need for wraparound and via holes. It reduces radiation loss. Since characteristic impedance is determined by the ratio of gap to conductor size in the line, size reduction is theoretically limited only by the fabrication process, though higher line losses will result $[13, \mathrm{p} .1]$. In addition, a ground plane exists between any two adjacent lines, hence cross-talk effects between adjacent lines is lower than with other types of lines $[13, \mathrm{p} .1]$. As a result, CPW circuits can be made denser 
than conventional microstrip circuits. Finally, transition from CPW to slotline is easier, allowing greater flexibility in the use of mixed transmission lines [14, p.375]. These advantages make CPW ideally suited for designing planar MMIC circuits.

In addition to design advantages, CPW offers manufacturing advantages. For example, CPW lends itself to the use of automatic pick-and-place and bond assembly equipments for surface-mount component placement $[13, \mathrm{p} .2]$. CPW also allows use of automated on-wafer measurement techniques for circuit characterization. These advantages make it cost effective to produce CPW based MMICs in large volume.

CPW does have some disadvantages. These include the creation of parasitic modes [14, p.375]. This is due to the fact that CPW can support even mode (typical) and odd mode (atypical) propagation. Thankfully, judicious use of air-bridges linking the ground-planes forces the waveguide to support only even mode propagation. Other disadvantages include lower power handling ability and field non-confinement [14, p.375]. Field nonconfinement means that for CPW half of the energy in the electric field is in air above the waveguide, while the other half is confined to the substrate, unlike in microstrip lines where the field is mostly confined to the substrate. This division of energies in CPW between air and the dielectric causes dispersion at higher frequencies, as well as causing interference problems due to radiated fields. 
The coplanar waveguide is a quasi-TEM waveguide, with the behavior of the electric fields for the even mode (typical) operation of the waveguide given in the Figure 2-7 below.

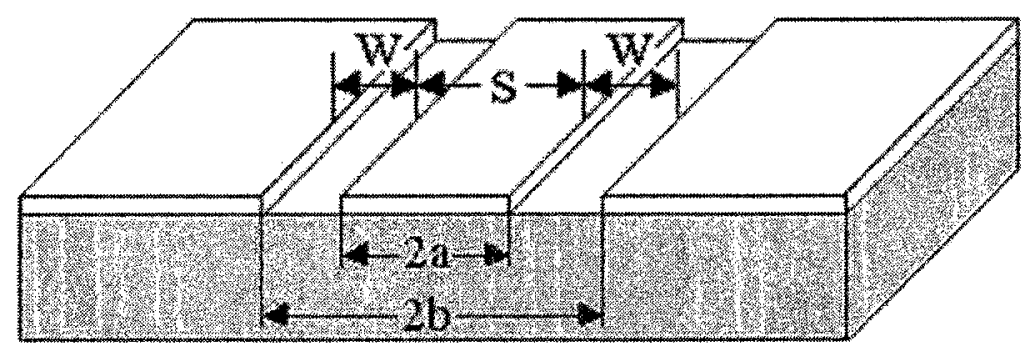

(a)

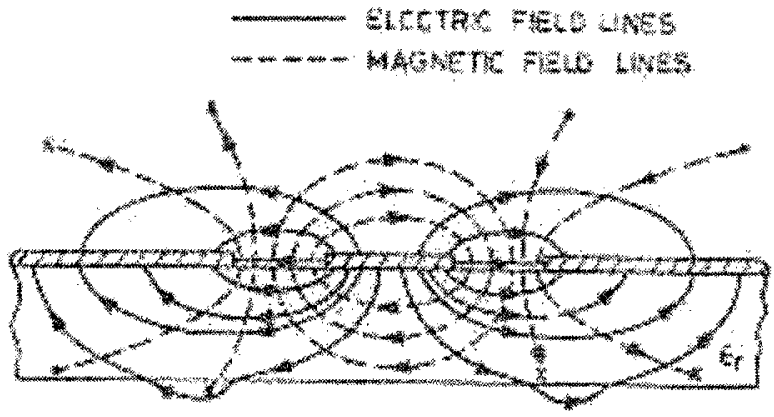

(b)

Figure 2-7: CPW showing (a) aspect ratio $a / b$, and (b) field distribution for even mode operation [14, p.376].

At higher frequencies, the mode of propagation in the CPW becomes non-TEM because longitudinal component of the magnetic field exists $[14$, p. 376].

Equations are given below for the characteristic impedance for CPW on a finite dielectric substrate, which is the case for the Phillips ${ }^{\mathrm{TM}}$ OMMIC ED02AH fabrication process used in this thesis: 


$$
Z_{C P W}=\frac{30 \pi}{\sqrt{\varepsilon_{e f f}}} \frac{K\left(k_{0}^{\prime}\right)}{K\left(k_{0}\right)}
$$

Where: $\varepsilon_{\text {eff }}=1+\frac{\left(\varepsilon_{r}-1\right)}{2} \frac{K\left(k_{1}\right)}{K\left(k_{1}^{\prime}\right)} \frac{K\left(k_{0}^{\prime}\right)}{K\left(k_{0}\right)}$

$$
\begin{aligned}
& \left.k_{0}=\frac{a}{b}=\frac{S}{S+2 W} \text { (Refer to Figure 2-7(a) above for } \mathrm{S} \text { and } \mathrm{W}\right) \\
& k_{0}^{\prime}=\sqrt{1-k_{0}^{2}} \\
& k_{1}=\frac{\sinh \left(\pi S / 4 h_{1}\right)}{\sinh \left\{[\pi(S+2 W)] / 4 h_{1}\right\}} \rightarrow \mathrm{h}_{1} \text { is the substrate thickness. } \\
& k_{1}^{\prime}=\sqrt{1-k_{1}^{2}} \\
& K\left(k_{1}\right), K\left(k_{1}^{\prime}\right) \text { are complete elliptic integrals. }
\end{aligned}
$$

Most planar microwave circuit designs require the use of resonant structures created with the use of stubs. The creation of short and open circuit resonant stubs in CPW is very easy. Figure 2-8 shows how to create a shorted and open-ended stub. 


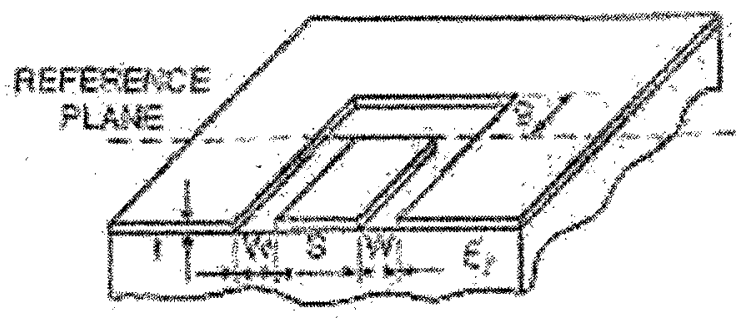

(a)

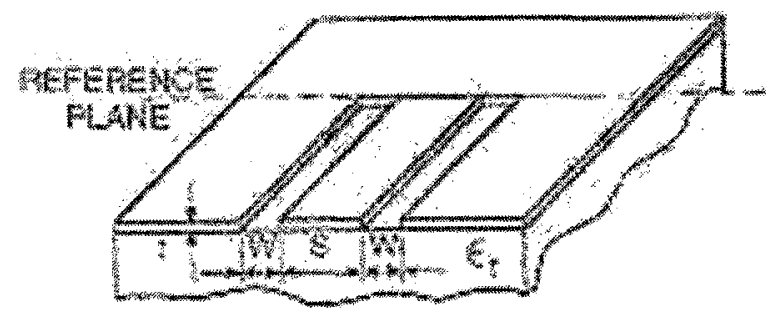

(b)

Figure 2-8: CPW (a) open and (b) short circuit stubs [14, p.443].

From Figure 2-8(a) above it is clear that for an open-circuit stub all that is required is that a gap exists between the end of the stub and the ground plane, while Figure 2-8(b) shows that for a short-circuit stub the stub needs to connect to the ground plane.

One advantage of CPW lines over planar transmission lines like microstrip is the ability to create both shunt and series resonant stubs. Figure 2-9 illustrates this. 


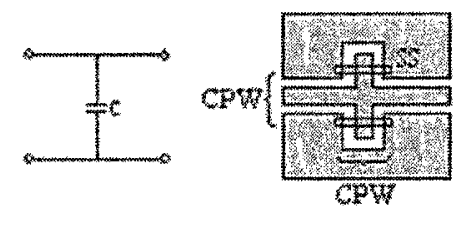

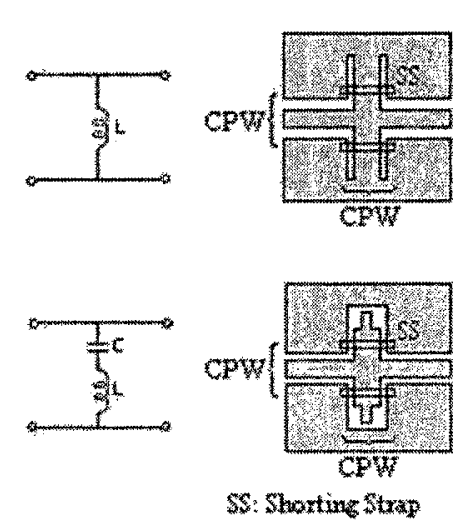

(a)

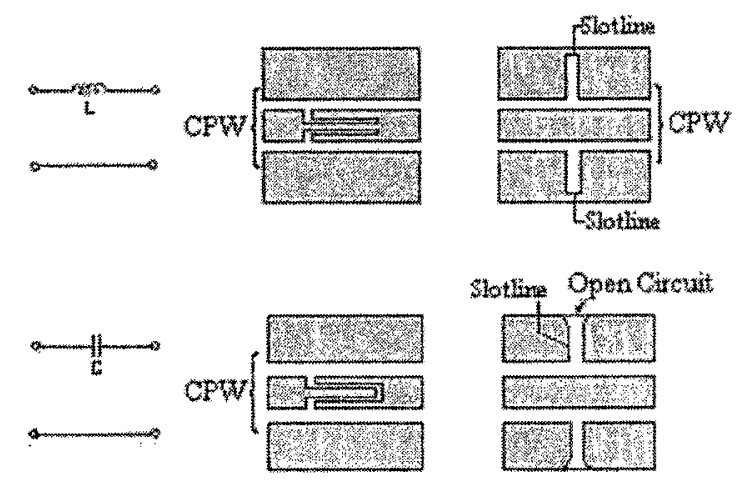

(b)

Figure 2-9: CPW (a) shunt and (b) series stub configurations [14, p.444].

Figure 2-9(a) above shows how to create in CPW, much as with microstrip lines, double shunt stub resonators. More interesting is how Figure 2-9(b) shows how series stub are created, shown in the center column, with an equivalent circuit using slotlines in the right-hand column and the symbolic representations for the circuit elements in the lefthand column. In microstrip circuits it is only possible to create shunt stubs. The ability to produce series resonant stubs gives designers an extra degree of freedom that helps to produce unique structures.

\subsubsection{Microstrip:}

The microstrip line is a common transmission line used in most high frequency circuits. It has been thoroughly researched over several decades, with well-defined closed-form expressions for calculating the characteristic impedance [10, p.162]. The microstrip line supports quasi-TEM modes of propagation. Similar to CPW lines, it is possible to create 
resonant stubs using microstrip lines, though for microstrip lines it is only possible to create shunt stubs. The figure $2-4$ below shows the microstrip line along with the field distribution for the microstrip line.

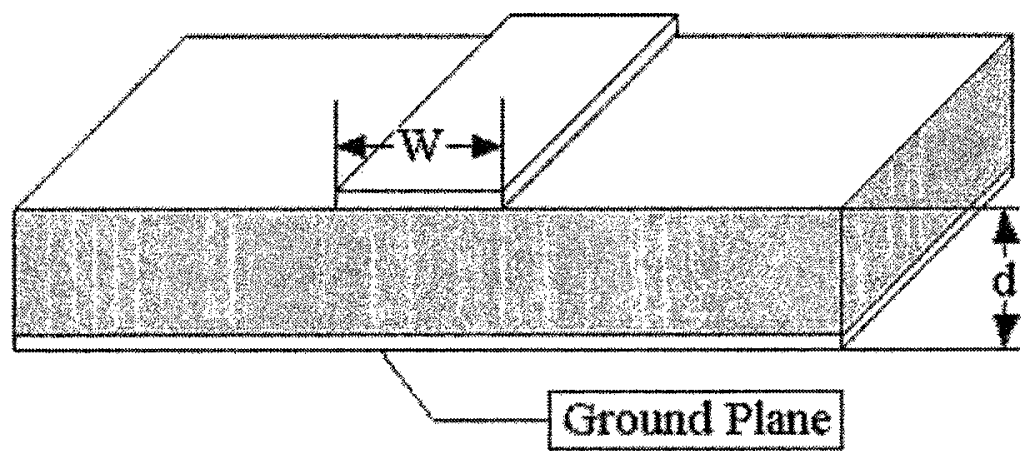

(a)

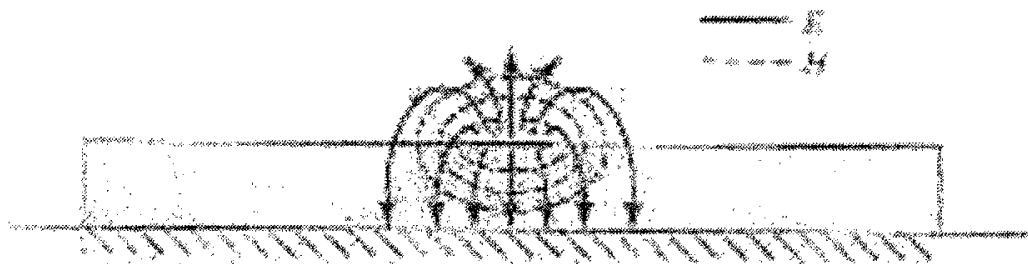

(b)

Figure 2-10 (a) microstrip line and (b) field distribution [10, p.161].

The fact that most of the fields are contained within the dielectric, as shown in the Figure 2-10(b) above, mean that static or quasi-TEM solutions are quite accurate for describing the wave characteristics such as phase velocity and propagation constant, as well as for extracting the characteristic impedance. The characteristic impedance can be calculated using the well-known closed-form expressions in [10, p.162]. 


\subsubsection{Slotline:}

Slotlines can be included in microstrip circuits by etching the slotline circuit in the ground plane of the substrate for coplanar circuits. This type of hybrid combination allows flexibility in the design of microwave circuits. Also, some circuits that cannot easily be created using microstrip lines, can be created using slotlines. These could be short circuits, high impedance lines, series stubs, and baluns [14, p.269]. The following Figure 2-11 shows a slotline and its field distribution.

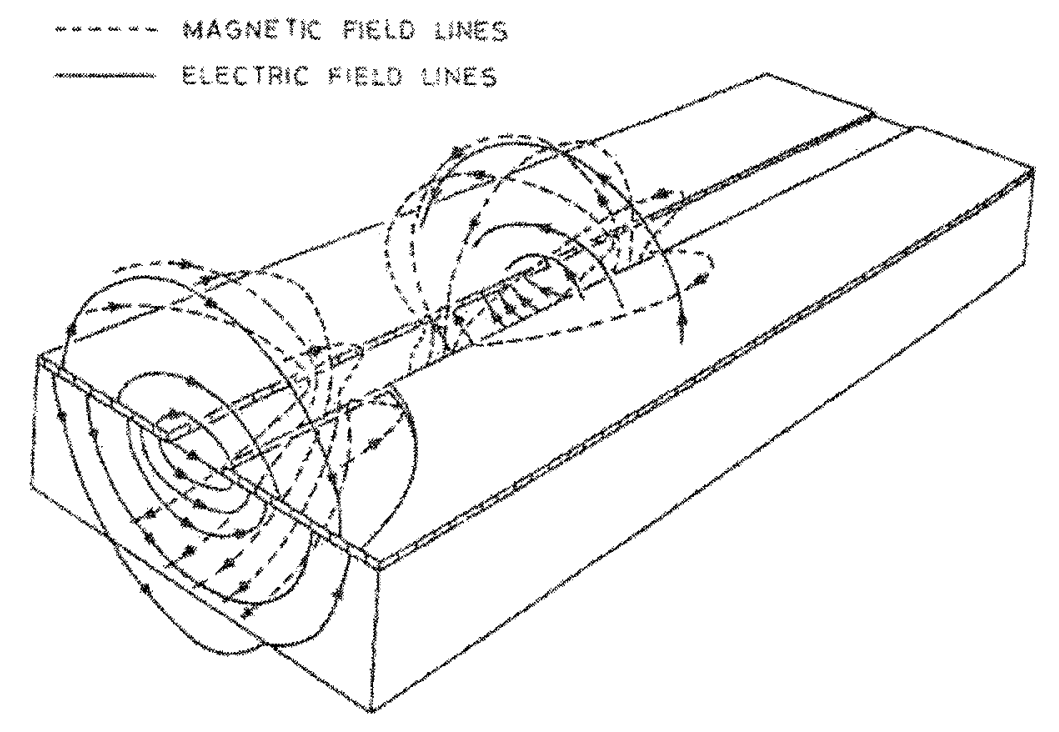

Figure 2-11: Slot line showing field distribution [14, p.270].

As seen in the Figure 2-11 above of a slotline, the wave propagates along the slot with the major electric field component oriented across the slot in the plane of metallization on the dielectric substrate. The mode of propagation is non-TEM and almost transverse electric (TE) in nature [14, p.269]. Because of this, static techniques cannot be applied to derive closed form expressions describing impedance and propagation characteristics of the slotline. The definition of $Z_{0 s}$, the characteristic impedance of the slotline, that is based 
on the power-voltage relationship can be used to give a general equation, and is found in $[14$, p.276]. For design equations with accuracy of about 2 percent, look to [14, p.283] for several closed-form expressions that depend on the aspect ration $\mathrm{W} / \mathrm{h}$, derived from curve fitting of empirical and numerical analysis.

As previously discussed for CPW and microstrip lines, slotlines can be used to design short and open circuit resonant stubs. Figure 2-12 shows the short-circuit stub.

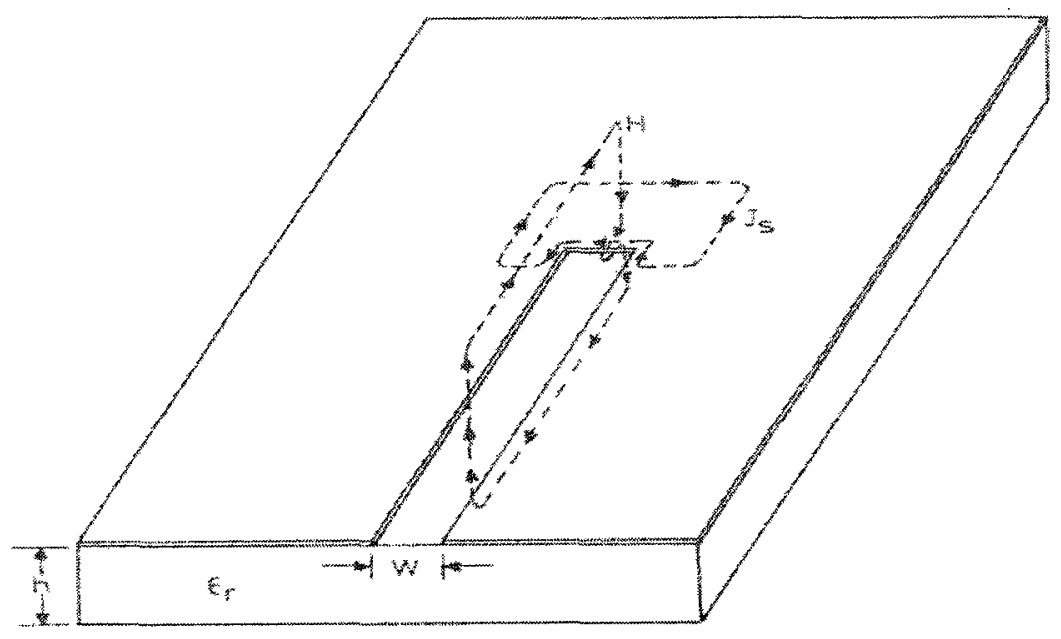

Figure 2-12: Slotline short-circuit stub [14, p.292].

The Figure 2-12 above shows that the short-circuit slotline stub just simply terminates in the ground plane. For the open-circuit stub, several options for termination are available, as illustrated in Figure 2-13 below. 


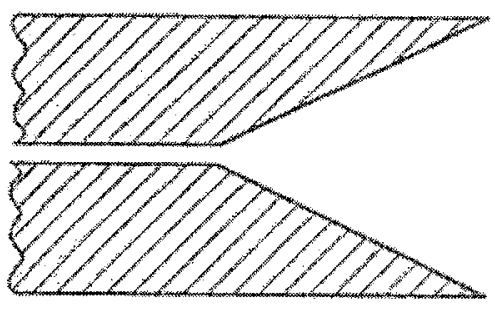

(a)

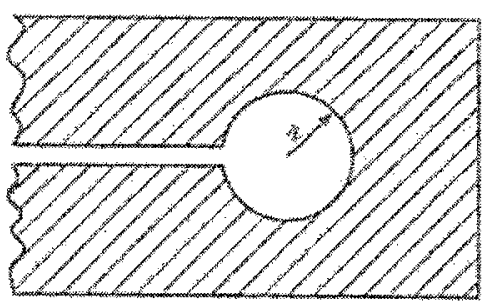

$(b)$

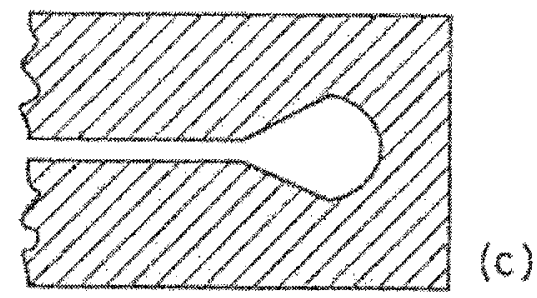

Figure 2-13: Slotline open-circuit stub with (a) a flared open end, (b) a circular disc open end, and (c) a combination of flared slot and halfdisc open end [14, p.295].

The different open-circuit terminations in Figure 2-13 above have varying degrees of performance, but mainly they offer flexibility when designing circuits.

\subsection{Comparison between CPW, microstrip, and slotline}

Having discussed three planar transmission lines, it is useful to summarize some of the differences between all three in table form. The following Table 2-2 indicates the typical upper and lower boundaries for the three types of transmission lines. 
Table 2-2: Limits of $Z_{0}$ for various transmission lines [14, p. 431].

\begin{tabular}{|lll|}
\hline Transmission line & $\begin{array}{l}\text { Lower Limit } \\
\text { for } \mathbf{Z}_{0}(\Omega)\end{array}$ & $\begin{array}{l}\text { Upper Limit } \\
\text { for } \mathbf{Z}_{\mathbf{0}}(\Omega)\end{array}$ \\
\hline & & \\
Microstrip & $11(\mathrm{~m})$ & $110(\mathrm{~d})$ \\
Slotline & $35(\mathrm{~d})$ & $250(\mathrm{~m})$ \\
Coplanar & $20(\mathrm{~m}, \mathrm{~d})$ & $250(\mathrm{~m}, \mathrm{~d})$ \\
Waveguide & & \\
\hline
\end{tabular}

In the Table 2-2 above, the letter ' $\mathrm{m}$ ' indicates the limitation is due to higher order modes, while the letter ' $d$ ' means that the limitation is due to physical dimensions [14, $p$. 431]. Some interesting points in the above table are that the microstrip line is the best choice for creating lower impedance lines, while CPW and slotline are better for creating high impedance lines.

Another table that gives qualitative comparisons between the three types of transmission lines is given below. 
Table 2-3: Qualitative comparison of various MIC lines [14, p. 434].

\begin{tabular}{|c|c|c|c|}
\hline Characteristic & Microstrip & Slotline & CPW \\
\hline $\begin{array}{l}\text { Effective dielectric constant } \\
\left(\varepsilon_{\mathrm{r}}=13 \text { and } \mathrm{h}=100 \mu \mathrm{m}\right)^{*}\end{array}$ & $\approx 8.6$ & $\approx 5.07$ & $\approx 7$ \\
\hline Power handling capability & High & Medium & Medium \\
\hline Radiation loss & Low & Medium & Medium \\
\hline Unloaded Q & Medium & Low & Medium \\
\hline Dispersion & Small & Large & Small \\
\hline $\begin{array}{l}\text { Mounting of Components: } \\
\text { In shunt configuration } \\
\text { In series configuration }\end{array}$ & $\begin{array}{l}\text { Difficult } \\
\text { Easy }\end{array}$ & $\begin{array}{l}\text { Easy } \\
\text { Difficult }\end{array}$ & $\begin{array}{l}\text { Easy } \\
\text { Easy }\end{array}$ \\
\hline Technological difficulties & $\begin{array}{l}\text { Ceramic holes } \\
\text { Edge plating }\end{array}$ & -- & -- \\
\hline $\begin{array}{l}\text { Elliptically polarized } \\
\text { magnetic field config. }\end{array}$ & Not available & Available & Available \\
\hline Dimensions & Small & Large & Large \\
\hline
\end{tabular}

* Relative permittivity taken from reference source.

\subsection{Unique Planar Structures}

Some excellent research on creating novel planar structures has been done by researchers at the Communications Research Centre. Some of the structures and techniques developed by K. Hettak were applied in this thesis. The following describes some structures that are used in later chapters to develop a novel planar coupler. 
Figure 2-14 shows a novel transition developed in January 2000.
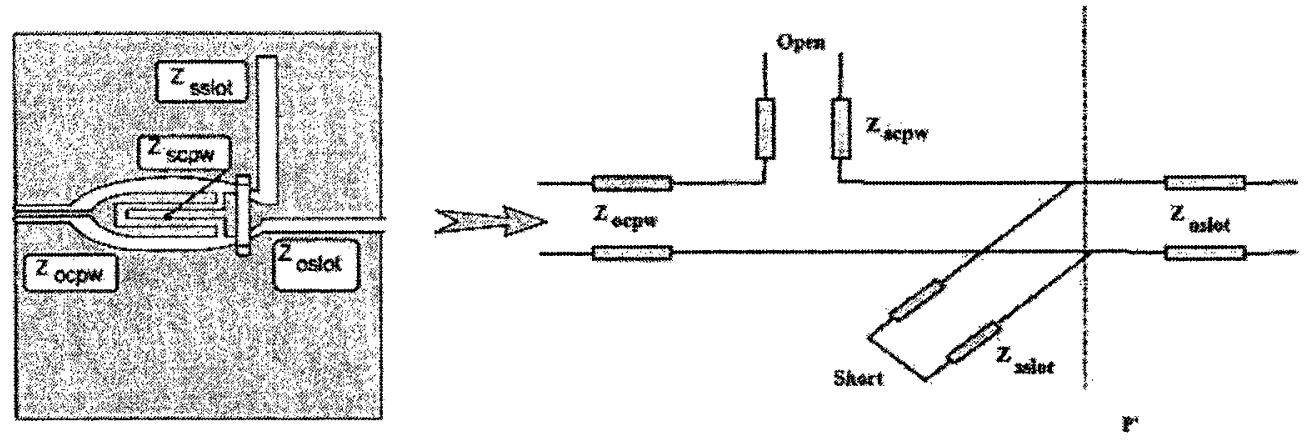

Figure 2-14: Novel CPW-to-Slotline transition [15].

The Figure 2-14 shows the layout and schematic representation of the transition. It consists of an open-circuit series resonant stub and a shunt short-circuit slot stub. These two stubs resonate to produce a wide bandwidth. The theory for how the stubs produce a wide bandwidth is discussed in the chapter 3 on designing a novel coupler, however suffice it to say that one of the design challenges is to make the characteristic impedance of the series resonant stub as small as possible, while making the shunt stub impedance as high as possible. 
The next figure is of another novel transition found in [15].

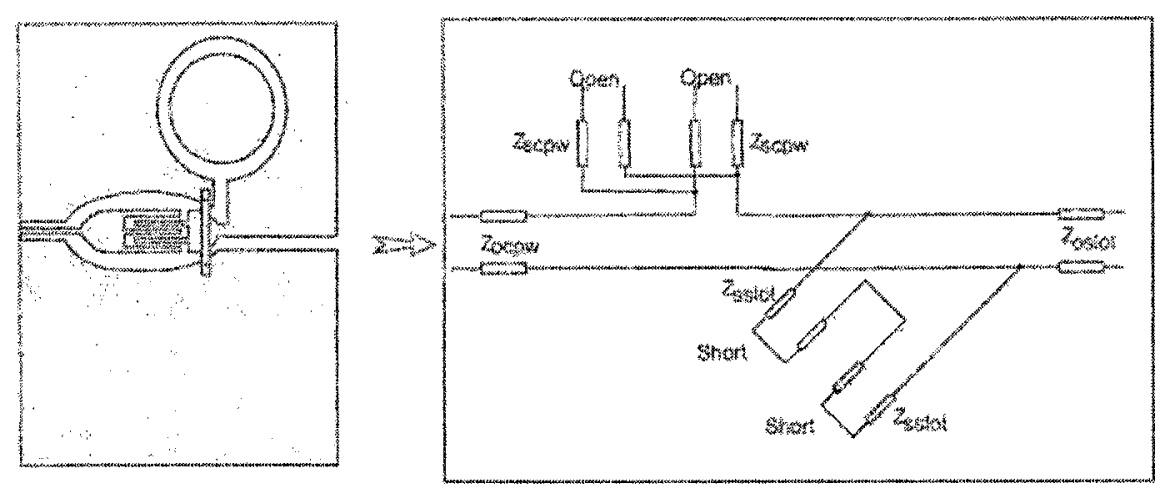

Figure 2-15: Second novel CPW-to-Slotline transition [15].

What distinguishes the Figure 2-15 above compared to the Figure 2-14 that was just discussed are the fact that there are two series stubs in parallel, and two shunt slotline stubs in series in the form of a ring. The effect of the parallel combination of series stubs is to halve the characteristic impedance, while the two shunt stubs in series increases the equivalent shunt impedance. These are two positive changes for creating a wider bandwidth.

The ring resonator in the Figure 2-15 above is actually from a family of resonators, given in the figure below. 


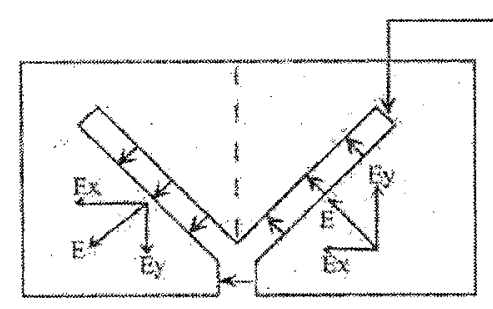

(a)

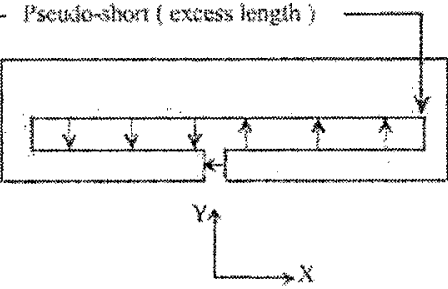

(b)

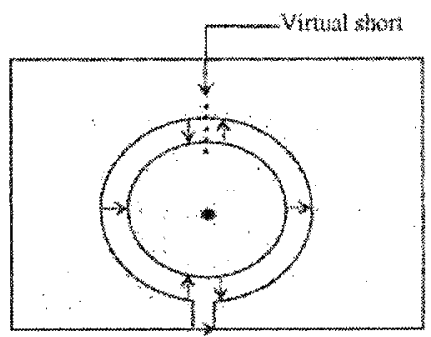

(c)

Figure 2-16: Electric Field distribution of three types of slotline resonators [15]

Figure 2-16(a) and Figure 2-16(b) are essentially the same, in that they are simply two shunt slotline stubs in series, albeit in different layout configurations. The layout of the two shunt slotline stubs in Figure 2-16(a) is such that it partially radiates. The layout of Figure 2-16(b) on the other hand is such that when the field is reflected back to the junction from both stubs, the fields arrive with a $180^{\circ}$ phase shift and thus cancel each other out, which stifles radiation [15]. Both Figures 2-16(a) and (b) have the problem that as frequency increases, the stubs' electrical length appears to "grow". This is what is meant by "excess length", and it is this property that makes the first two configurations of Figure 2-16 narrowband [15]. The same is not true for the ring resonator in Figure 216(c) because regardless of frequency, the energy is split and reaches the top point at opposite phases, canceling each other out and creating a virtual short [15]. Thus the ring resonator has a broader bandwidth than the other two.

At this point, the tools needed to design a new coupler have been described. The next chapter describes in detail the design of the new coupler. 


\section{CHAPTER 3: DESIGN OF PLANAR COUPLER}

In the previous two chapters, the groundwork for designing a new coupler was laid, including an examination and comparison of existing couplers, an overview of different transmission lines, and an exploration into novel techniques for creating unique planar structures. This chapter details the steps taken to achieve the final design of the new coupler. The first section discusses the underlying theory. The sections following provide a design progression, explaining the reasons for various design iterations and providing simulated results.

\subsection{Design evolution and theory}

The task of this thesis was to investigate whether a novel, broadband coupler could be created using a well-known coplanar T-junction along with some novel resonant circuits. A common T-junction, drawn as a coplanar circuit, is shown in Figure 3-1 below.

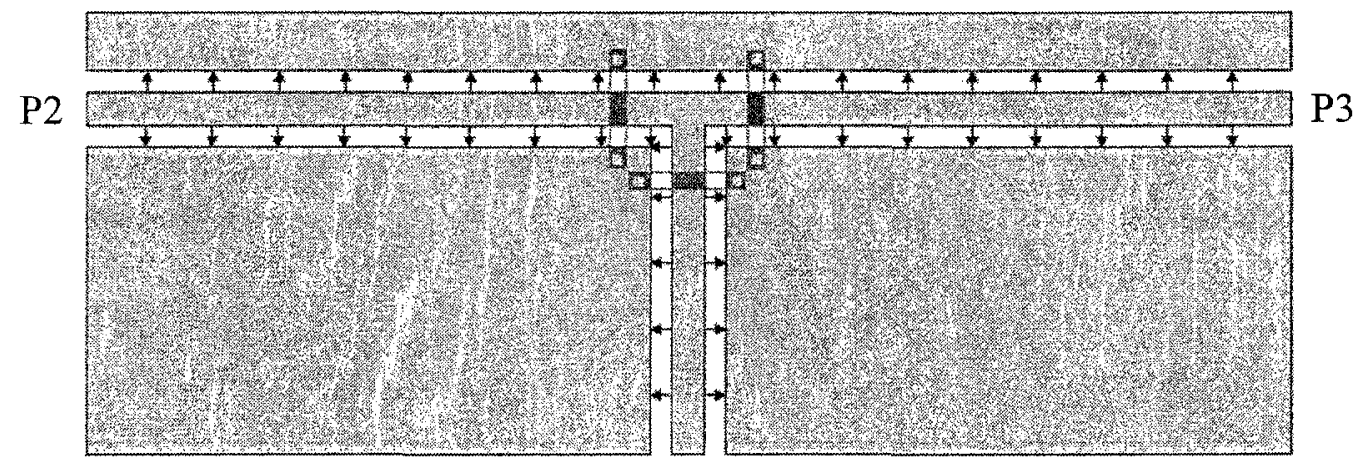

P1

Figure 3-1: Common coplanar T-junction. 
In Figure 3-1, the gray area represents the top metallization layer, which contains the conductor and ground plane for the coplanar line. The white tracks represent the gaps between the conductor and ground plane, through which the propagating wave passes. The red, or dark gray layer represents a lower metallization layer used for creating airbridges. Figure $3-1$ shows that the T-junction has three ports. It is in fact a loss-less, reciprocal three-port device - a fact that makes matching the T-junction difficult, and will be discussed in more detail later [16]. The arrows in Figure 3-1 indicate which way the electric field is pointing in the coplanar slots. The above T-junction effectively splits the power entering port 1 evenly between port 2 and port 3 . The output signals of the Tjunction are in phase, however it is desirable that the new coupler have a $180^{\circ}$ phase split between the output ports - a useful feature in most of the circuits that use couplers. A $180^{\circ}$ phase shift can conveniently be introduced in the coplanar T-junction as follows:

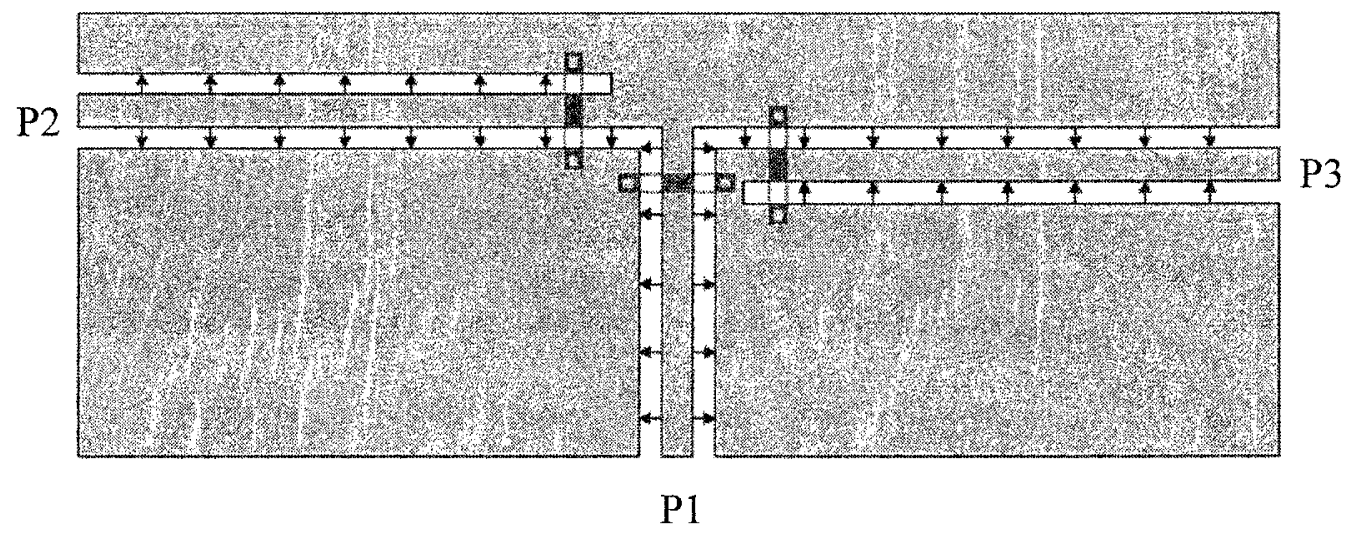

Figure 3-2: Coplanar T-junction with $180^{\circ}$ phase split between output ports.

Figure 3-2 shows that by 'flipping' one of the output lines and enforcing polarity with air bridges, a $180^{\circ}$ phase shift is created as indicated by the reversal in the direction of the arrows in port 3 . This method for creating a $180^{\circ}$ phase shift is not a new idea, but it will 
be a key component for making the new coupler broadband. I he reason tor this is because the phase shift is inherent; it does not depend on the electrical lengths of structures within the coupler, which depend on frequency, as is the case for the common couplers previously discussed, as well as for various baluns such as the Marchand balun. The implication of this is that if not for other factors - to be addressed later in the design - this structure could theoretically have a $180^{\circ}$ phase shift over an infinite bandwidth!

The factors limiting the bandwidth of this structure are:

- Parasitic junction reactances exacerbated by sharp edges and corners.

- Change from CPW-Slotline-CPW in the T-junction; this is due to the change in modes from TEM to TE to TEM again.

Parasitic junction reactances can be modeled as in Figure 3-3 below:

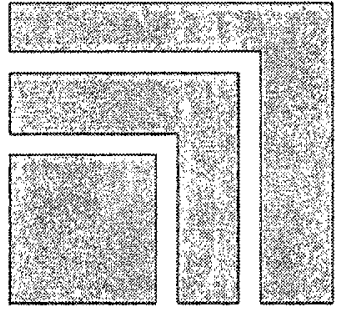

(a)

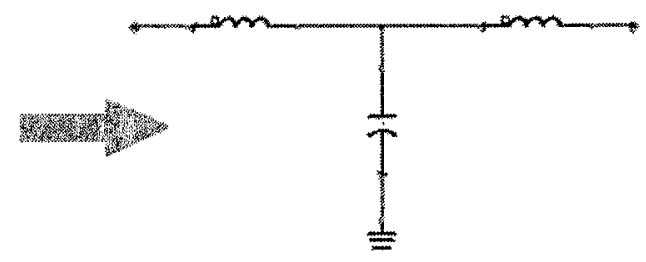

(b)

Figure 3-3: Model of sharp bend in coplanar line.

Figure 3-3 shows that a sharp bend in a coplanar transmission can be modeled at high frequency with a lumped capacitor in the center and two inductors at the ports. The same model applies to other planar transmission lines. Figure 3-3(b) is essentially a low-pass filter. Since the amount of parasitic capacitance is relatively low, the effects of the bend 
only become evident at very high frequencies, however they do reduce the upper frequency bound of the circuit. Figure 3-4 shows how one can reduce the effects of parasitic capacitance.

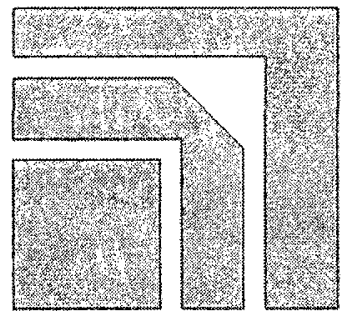

(a)

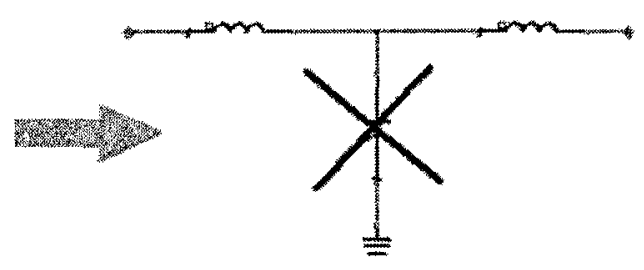

(b)

Figure 3-4: Model of modified bend for reducing parasitic reactance.

Figure 3-4(a) shows that by carving off some of the metal in the conductor at a sharp corner, the parasitic capacitance can be cancelled out, or at the very least reduced to improve the circuit bandwidth.

The change from coplanar to slotline that occurs in the $\mathrm{T}$-junction also reduces the bandwidth because these transmission lines support different wave modes. In simple terms, these different modes represent a change in characteristic impedance, and this mismatch - however small - reduces bandwidth. The best way to minimize this effect is to reduce the length of slotline as much as possible. The shorter the length of the discontinuity, the less effect it has on the bandwidth.

The first thing that must be addressed in the coupler design are the CPW slots on the output ports left dangling because they are unused, while the other slot is connected to the T-junction. This is illustrated in Figure 3-5 below: 


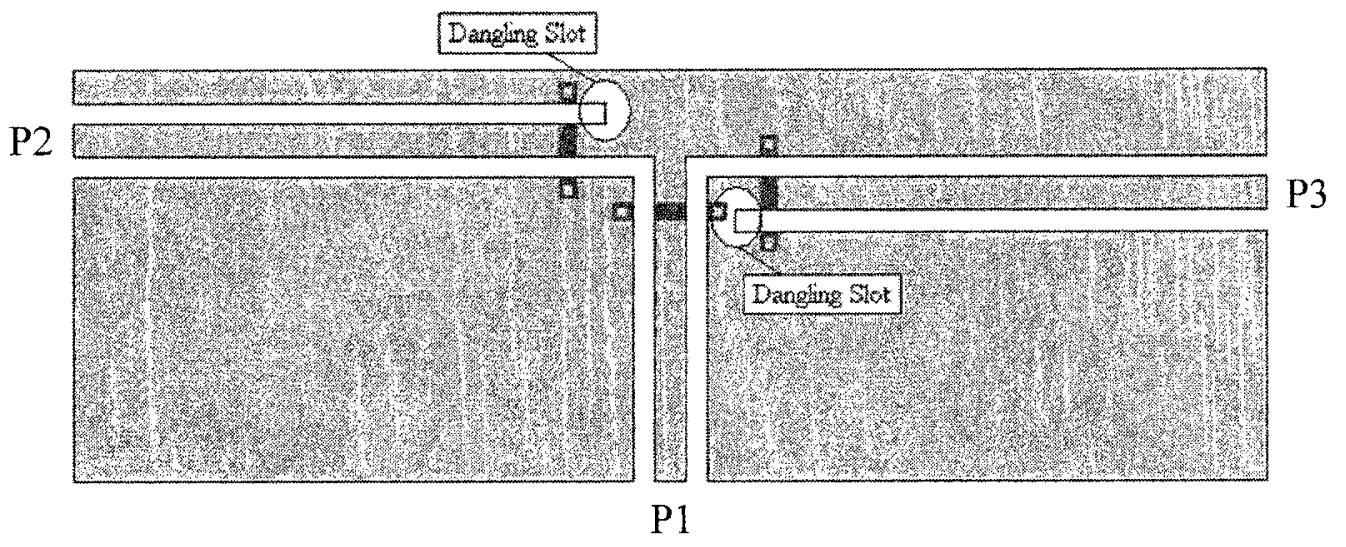

Figure 3-5: T-junction with dangling slots.

These dangling slots must be terminated ideally with an open circuit. The reason for this is because if left unterminated, upon reflecting off the conducting boundary and reversing polarity the field will cancel itself out because polarity is enforced by the air bridge, thus turning the coupler into an effective attenuator. By terminating the CPW slot with an open circuit, there is no reversal of polarity and thus the field is maintained. In reality, an open circuit is created by using a short circuit quarter-wavelength stub. Shunt slotline stubs are used to terminate one slot of the coplanar waveguide. Figure 3-6 below illustrates this addition: 


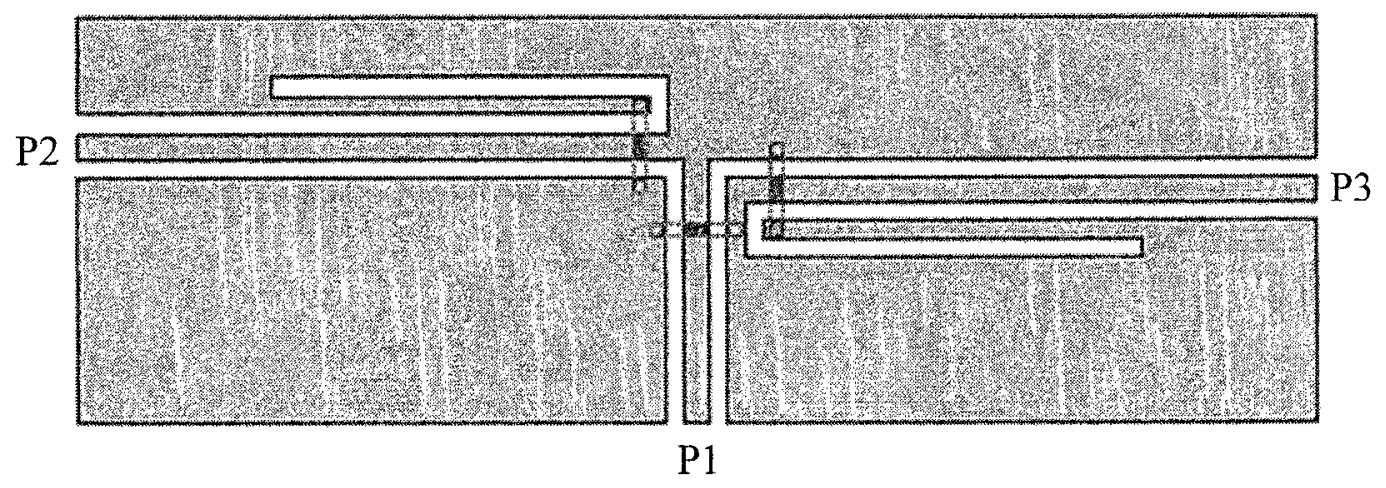

Figure 3-6: T-junction with terminated slots.

Figure 3-6 is effectively a combination of a CPW-Slotline T-junction plus two CPW-

Slotline transitions, which is modeled more clearly in Figure 3-7 below:

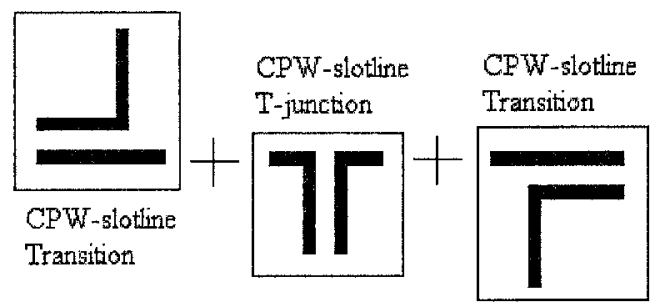

Figure 3-7: CPW-slotline Transitions with CPW-Slotline T-junction.

In Figure 3-7, the white space represents the top metallization layer, while the dark tracks are the CPW gaps and slotline gaps. The focus of the design is pointed to improving the response of the CPW-Slotline transitions. Much experimental work has been performed by Khelifa Hettak [15] in designing different variations of the CPW-Slotline transition, as discussed in chapter 2. His techniques are applied here in the design of the new coupler.

Improvement to the CPW-Slotline transition begins with an equivalent circuit of the most basic CPW-Slotline transition, shown in Figure 3-8: 


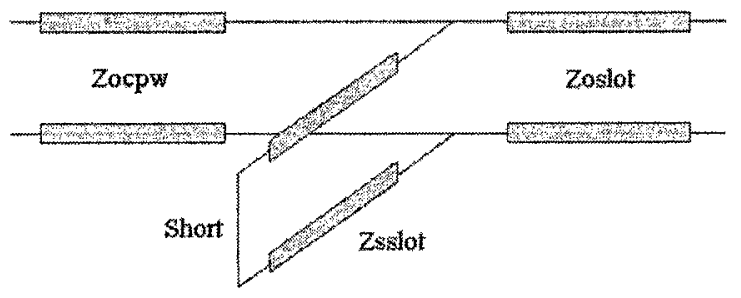

(a)

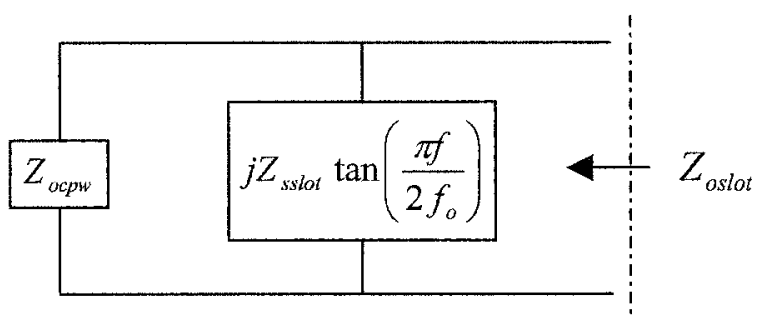

(b)

Figure 3-8: (a) Equivalent circuit and (b) block diagram of CPW-Slotline transition.

Figure 3-8(a) shows that the basic CPW-Slotline transition consists only of a shunt slotline stub. For large bandwidths, $Z_{\text {OSLOT }}$ must equal $Z_{O C P w}$. This is not possible in the circuit in Figure 3-8(a), for the following reason [15]:

$$
\begin{aligned}
Z_{\text {OSLOT }} & =Z_{\text {OCPW }} / /\left[j Z_{\text {SSlot }} \operatorname{Tan}(\phi)\right] \\
& =\frac{Z_{O C P W}\left[j Z_{\text {SSlot }} \operatorname{Tan}(\phi)\right]}{Z_{O C P W}+j Z_{\text {SSlot }} \operatorname{Tan}(\phi)}
\end{aligned}
$$

Where: $Z_{\text {OSLOT }}$ is the impedance seen looking into the transition and is also ideally equal to the characteristic impedance of the sloline $\mathrm{Z}_{\mathrm{OCPW}}$ is the CPW characteristic impedance $Z_{\text {SSlot }}$ is the characteristic impedance of the shunt slotline stub

For a perfect match throughout the transition structure, $\mathrm{Z}_{\mathrm{OSLOT}}$ must equal $\mathrm{Z}_{\mathrm{OCPW}}$.

However, since $Z_{O S L O T}$ from equation 3-1 is clearly a complex number, it cannot equal 
$\mathrm{Z}_{\mathrm{OCPW}}$, which is a purely real number. Therefore a matching structure is required between the two transmission lines. This is achieved through the addition of a resonant structure such that the imaginary components of $Z_{\text {OSLOT }}$ cancel out. This can be done by adding a series open-circuit stub to the transition, as illustrated in Figure 3-9 below:

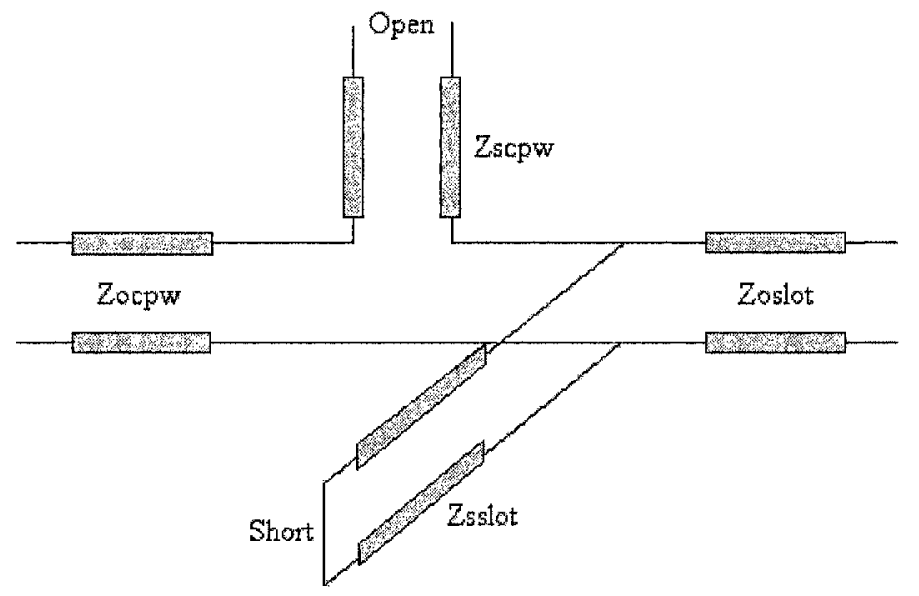

Figure 3-9: Series stub added to the equivalent circuit.

Adding a series stub is easy to do in coplanar circuits, and presents opportunities for creating a number of creative structures [15]. A quarter-wavelength open-circuit stub is selected because at the center frequency for which the stub is designed, the impedance at the input of the stub will be zero; i.e. it behaves as a short and thus does not add impedance to the CPW line, which is important to keep constant over frequency. The block diagram for Figure 3-9 is given in Figure 3-10: 


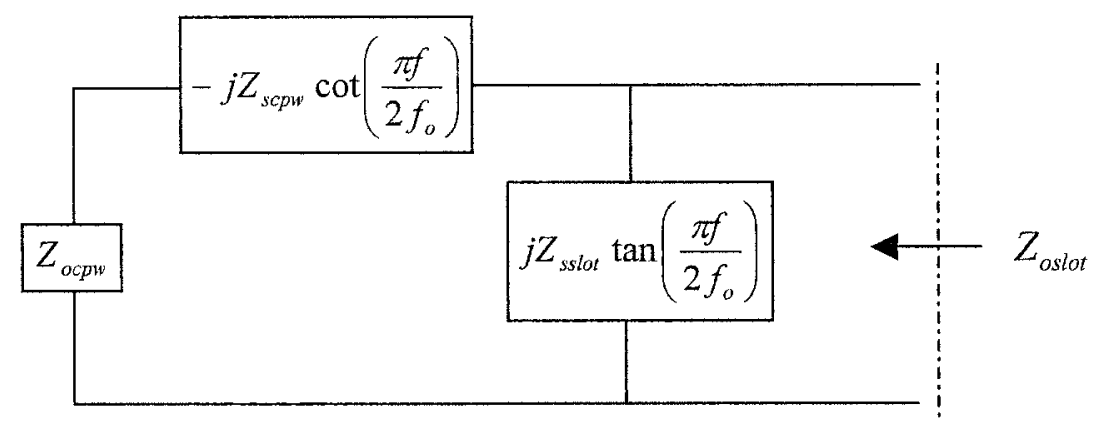

Figure 3-10: Block diagram for the transition with a series stub.

$Z_{\text {OSLOT }}$ is calculated for the new transition circuit as follows [15]:

$Z_{\text {OSLOT }}=Z_{c} / /\left[Z_{a}+Z_{b}\right] \quad$ (Arbitrary impedances in parallel)

$$
\begin{aligned}
& =\frac{Z_{c}\left[Z_{a}+Z_{b}\right]}{Z_{a}+Z_{b}+Z_{c}} \\
& =\frac{\left(j Z_{S S l o t} \operatorname{Tan}(\phi)\right)\left[Z_{O C P W}-j Z_{S C P W} \cot (\phi)\right]}{\left[Z_{O C P W}-j Z_{S C P W} \cot (\phi)\right]+j Z_{S S l o t} \operatorname{Tan}(\phi)} \\
\therefore Z_{\text {OSLOT }} & =\frac{Z_{O C P W}\left[1-j \frac{Z_{S C P W}}{Z_{O C P W}} \cot (\phi)\right]}{\left[1-j \frac{Z_{O C P W}}{Z_{S S l o t}} \cot (\phi)-\frac{Z_{S C P W}}{Z_{S S l o t}} \cot ^{2}(\phi)\right]}
\end{aligned}
$$

Where: $Z_{\mathrm{SCPW}}$ is the characteristic impedance of the series stub.

All other impedances are same as in equation 3-1. 
For $\mathrm{Z}_{\mathrm{OSLOT}}=\mathrm{Z}_{\mathrm{OCPW}}$ to be true:

$$
\left[1-j \frac{Z_{S C P W}}{Z_{\text {OCPW }}} \cot (\phi)\right]=\left[\left(1-\frac{Z_{S C P W}}{Z_{\text {SSlot }}} \cot ^{2}(\phi)\right)-j\left(\frac{Z_{O C P W}}{Z_{\text {SSlot }}} \cot (\phi)\right)\right]
$$

Therefore for the widest bandwidth the following two expressions must be true:

(i) $\frac{Z_{S C P W}}{Z_{\text {SSlot }}} \rightarrow 0$ i.e. A high $Z_{\text {SSlot }}$ impedance and a low $Z_{S C P W}$ impedance are required.

(ii) $\frac{Z_{S C P W}}{Z_{O C P W}}=\frac{Z_{O C P W}}{Z_{S S l o t}} \rightarrow$ Rearranged in terms of $Z_{S C P W}: Z_{S C P W}=\frac{Z_{O C P W}^{2}}{Z_{S S l o t}}$

Substituting the equation for $\mathrm{Z}_{\mathrm{SCPW}}$ into equation 3-2 yields the following:

$$
Z_{\text {OSLOT }}=\frac{Z_{O C P W}\left[1-j \frac{Z_{\text {OCPW }}}{Z_{\text {SSlot }}} \cot (\phi)\right]}{\left[1-\left(\frac{Z_{\text {OCPW }}}{Z_{\text {SSlot }}}\right)^{2} \cot ^{2}(\phi)-j\left(\frac{Z_{\text {OCPW }}}{Z_{\text {SSlot }}}\right) \cot (\phi)\right]}
$$

From equation 3-4 it is clear that $Z_{\text {OSLOT }}$ will equal $Z_{O C P W}$ when $\frac{Z_{\text {OCPW }}}{Z_{\text {SSlot }}}$ tends toward zero.

Since $Z_{\mathrm{OCPW}}$ is chosen to be fixed at $50 \Omega$, this would require $Z_{\text {SSlot }}$ to be very high. This could prove difficult to achieve because to increase the characteristic impedance of a 
slotline requires an increase in the width of the slot, and there is only so much the width can increase before the slotline suffers from multi-mode issues. This is true because at some point there is a danger of the slot becoming large enough for resonance to occur across it for frequencies within the band of interest. This occurs for a width of $\mathrm{w}>$ $0.25 \lambda_{0}[14, p .277]$. It is also difficult to create a design based on changing $Z_{0}$ for a slotline. This is due to the fact that slotlines support quasi-TE modes that have no natural closed form expressions for $Z_{0}[14]$.

Taking a different approach, the expression for $Z_{S S l o t}$ is substituted in the expression for Z OSLOT, as follows:

$$
Z_{\text {SSlot }}=\frac{Z_{O C P W}^{2}}{Z_{\text {SCPW }}}
$$

Which yields,

$$
Z_{\text {OSLOT }}=\frac{Z_{O C P W}\left[1-j \frac{Z_{S C P W}}{Z_{O C P W}} \cot (\phi)\right]}{\left[1-j \frac{Z_{S C P W}}{Z_{O C P W}} \cot (\phi)-\left(\frac{Z_{S C P W}}{Z_{O C P W}}\right)^{2} \cot ^{2}(\phi)\right]}
$$

Now, rather than an expression which requires manipulation of $\mathrm{Z}_{\mathrm{SSlot}}$, equation 3-5 requires manipulation of $Z_{S C P W}$. More specifically, it is necessary to make $Z_{S C P W}$ - the characteristic impedance of the series stub - as close to zero as possible, while still 
having the effect of canceling out the reactive component in the expression for $Z_{\text {OSLOT. }}$.

Doing so will eliminate all of the terms involving $\frac{Z_{S C P W}}{Z_{O C P W}}$, leaving $\mathrm{Z}_{\mathrm{OSLOT}}=\mathrm{Z}_{\mathrm{OCPW}}$.

So the design of the CPW-Slotline transition still requires a slotline stub of one quarterwavelength to terminate the unused CPW slot, however its characteristic impedance is fixed to $50 \Omega$. The slotline can be manipulated to achieve better cancellation of the propagating field, creating a better open-circuit termination. To improve the bandwidth, the stucture of the series stub must be designed such that the lowest possible $Z_{\mathrm{SCPW}}$ is achieved.

\subsection{Transition and Coupler designs}

The following paragraphs describe various designs of CPW-Slotline transitions that were simulated in Agilent's Momentum ${ }^{\mathrm{TM}}$ RF passive circuit simulator that comes packaged in Agilent's Advanced Design System ${ }^{T M}$ (ADS) software. The transitions were optimized for a center frequency of $30 \mathrm{GHz}$ and measured over a frequency span from $20 \mathrm{GHz}$ to 40 $\mathrm{GHz}$, which is the band of interest for this thesis for reasons given in the introduction.

Once the transitions were optimized, they were applied in a coupler configuration (refer to Figure 3-6), thus producing the various coupler designs created for this thesis, ultimately leading to the final coupler design. 


\subsubsection{Transition \#1 design:}

The first transition design is exactly as depicted in the Figure 3-11. In this figure the white space represents the CPW and Slotline gaps, while the gray area is the top metallization layer containing the conductor and ground plane of the CPW lines. Figure 3-11 is the most basic transition, consisting only of the slotline stub necessary to terminate the unused slot of the CPW line without the addition of a series stub for widening the bandwidth. For simulation purposes - i.e. to use the same ports at both ends of the structure - the transition designs are always simulated as two of the same type of transition placed back-to-back. Figure 3-9 shows the first transition design as it was laid out for simulation in Momentum ${ }^{\mathrm{TM}}$.

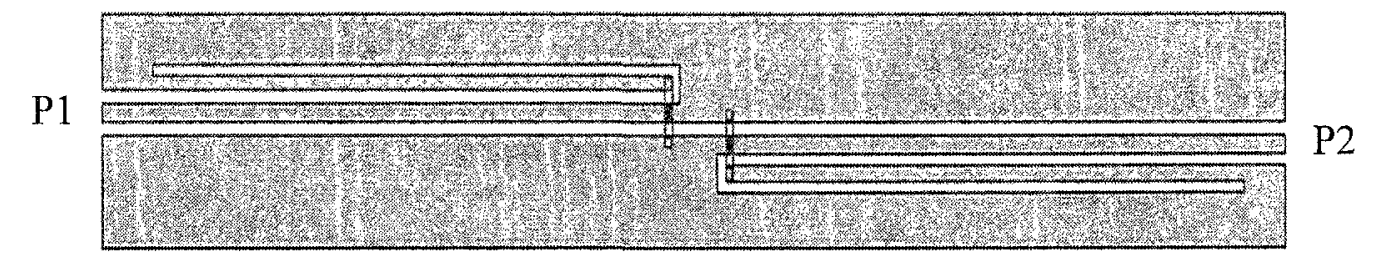

Figure 3-11: Back-to-back layout of Transition \#1

The slotline stubs in Figure 3-11 were tuned in order to be a quarter-wavelength long at $30 \mathrm{GHz}$, which is the center of the $20 \mathrm{GHz}$ band of interest. The slotline stubs are 850 $\mu \mathrm{m}$ long, and the width of the slotline is the same width as the slot in the CPW line -13 $\mu \mathrm{m}$. The above transition was simulated in Momentum $^{\mathrm{TM}}$ from $20 \mathrm{GHz}$ to $40 \mathrm{GHz}$ using an adaptive algorithm to produce smooth curves. The results of this simulation are provided in Figure 3-12. 


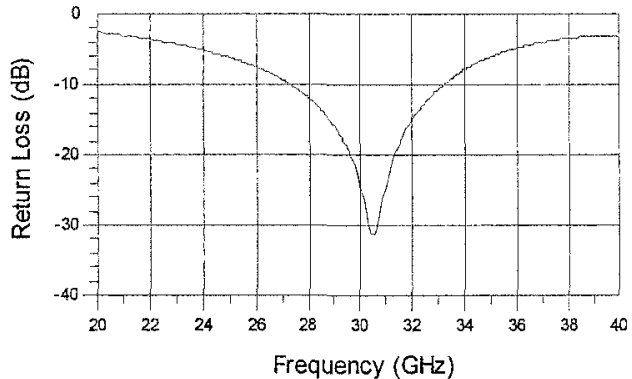

(a)

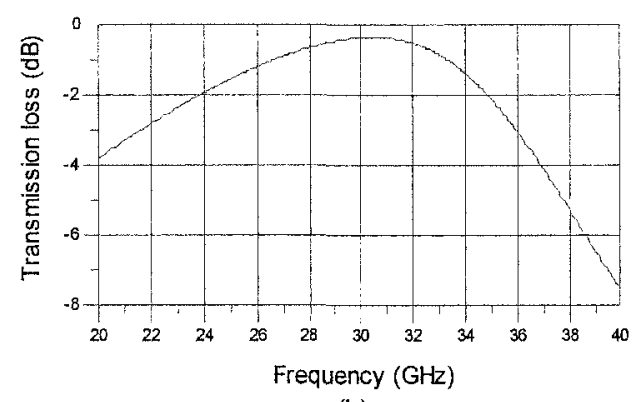

(b)

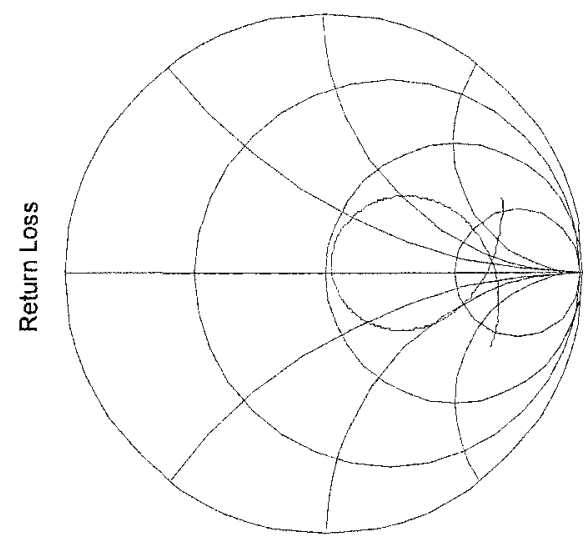

freq $(20.00 \mathrm{GHz}$ to $40.00 \mathrm{GHz})$

(c)

Figure 3-12: Simulation results for Transition \#1.

Of most importance to note is that Figure 3-12(a) shows distinctly the sharp dip in reflection coefficient at the center frequency of $30 \mathrm{GHz}$. Similarly illustrative is the Smith chart plot of the S11 values, in Figure 3-12(c), over frequency. On the Smith chart the curve approaches closer to the center of the chart - i.e. the $50 \Omega$ point of perfect match - then pulls away from the center as frequency moves beyond $30 \mathrm{GHz}$. The ideal scenario would be to have the various frequency points circle tightly around the center of the chart, producing very low reflection coefficients over as wide a band as possible. The structure, as it was simulated, produced a bandwidth (referenced to $-10 \mathrm{~dB}$ ) of $6.5 \mathrm{GHz}$, or $16.25 \%$. 


\subsubsection{Coupler \#1 design:}

Having tuned the design for Transition \#1, Transition \#1 was then used in a coupler design as illustrated in Figure 3-13 below:

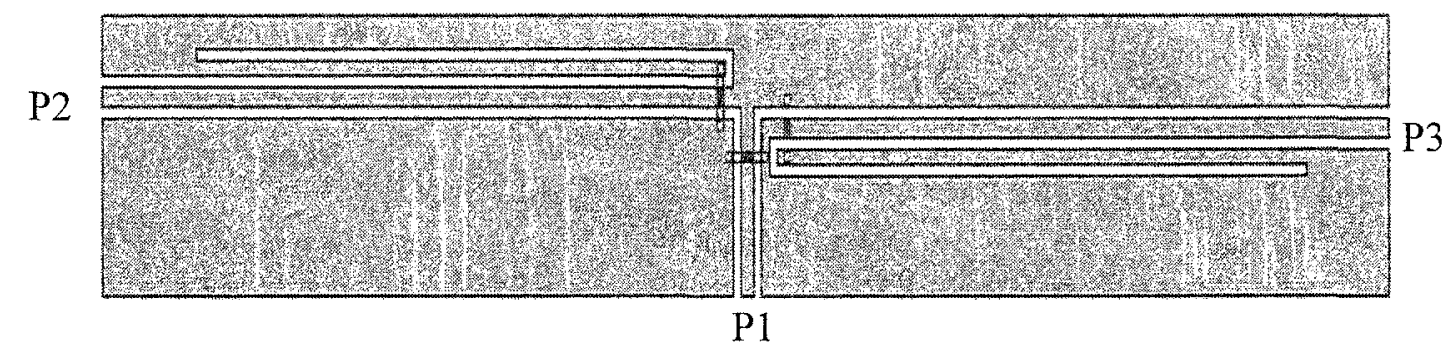

Figure 3-13: Coupler \#1

The coupler was simulated over a frequency range from $20 \mathrm{GHz}$ to $40 \mathrm{GHz}$, just as for the transition. Figure 3-14 gives the results for this simulation. 


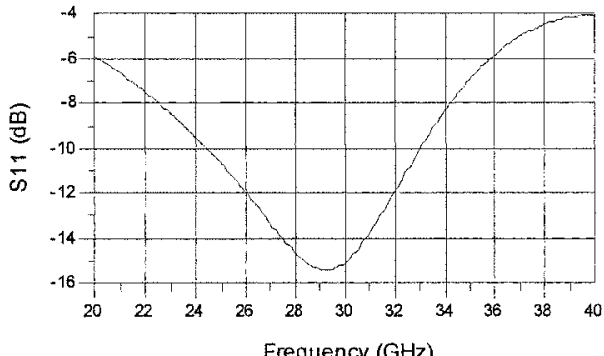

(a)

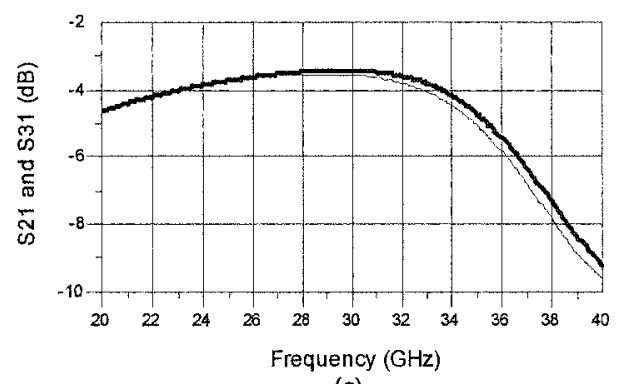

(c)

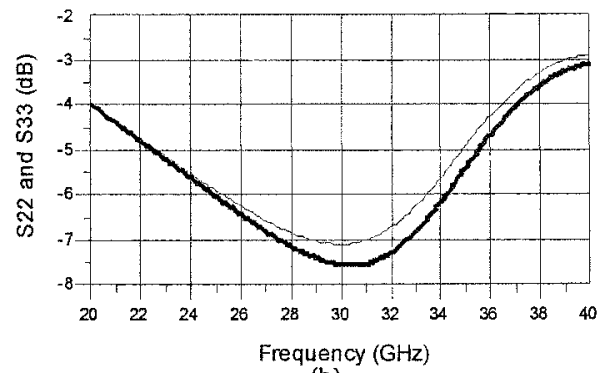

(b)

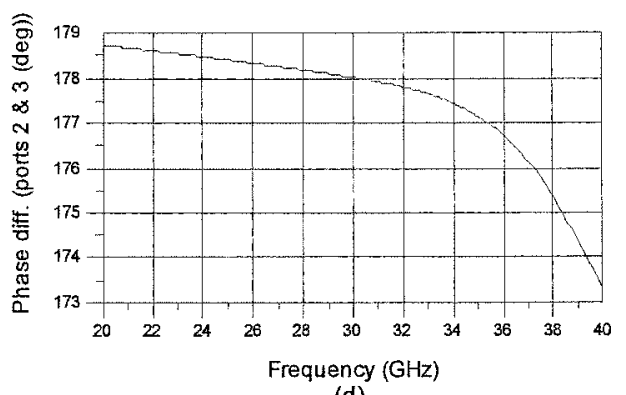

(d)

Legend:

Thin line - Port 2

Thick line - Port 3

Figure 3-14: Simulation results for Coupler \#1.

In Figure 3-14, note that the ports 1, 2, and 3 are optimally matched at $30 \mathrm{GHz}$. Of most importance is the difference in phase between port 2 and port 3 . This phase difference must be $180^{\circ}$, within $1^{\circ}$ or $2^{\circ}$, over as wide a frequency range as possible. The graph of Figure 3-14(d) shows the difference in phase from $20 \mathrm{GHz}$ to $40 \mathrm{GHz}$ falls between $179^{\circ}$ and $175.5^{\circ}$. This is a good reference for further designs, as the phase difference remains within $5^{\circ}$ of $180^{\circ}$ over the entire $20 \mathrm{GHz}$ band of interest. 


\subsubsection{Transition \#2 design:}

The next transition design includes a series stub to take advantage of the resonance effects discussed in the theory earlier. The length and width of the series stub, along with the length of the slotline stub were manipulated to give the best bandwidth for this design. The length of the series coplanar stub is $900 \mu \mathrm{m}$, and its width is $20 \mu \mathrm{m}$. The length of the shunt slotline stub remains $850 \mu \mathrm{m}$. Figure 3-15 shows Transition \#2.

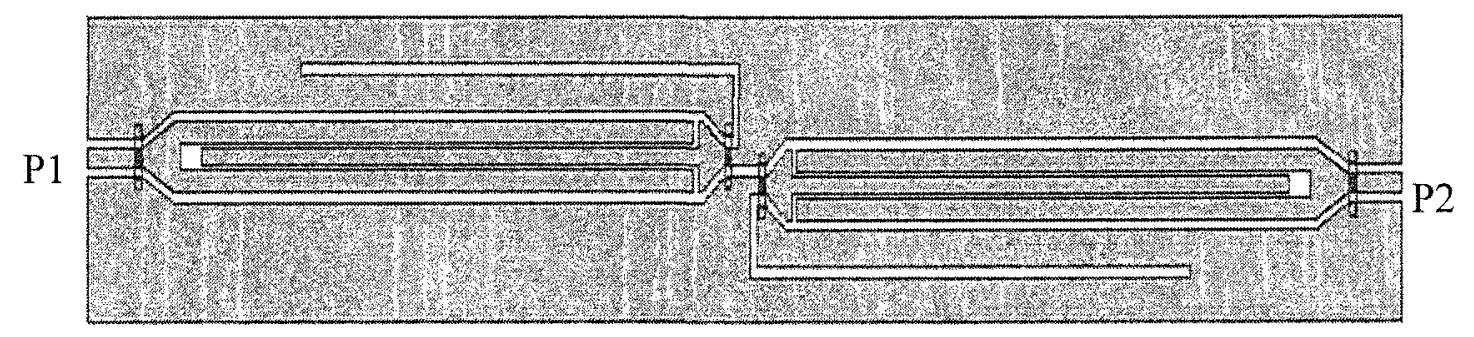

Figure 3-15: Transition \#2

The above design was simulated in Momentum ${ }^{\mathrm{TM}}$, giving the following results. 


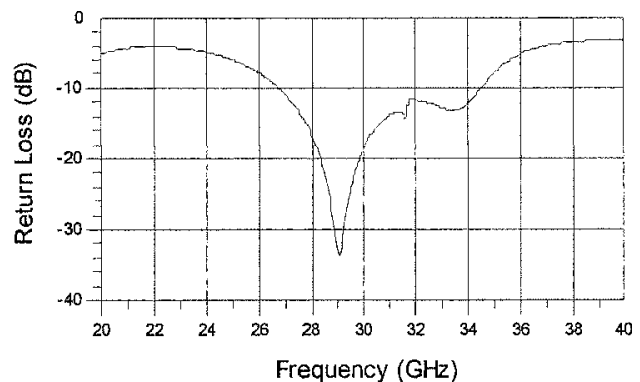

(a)

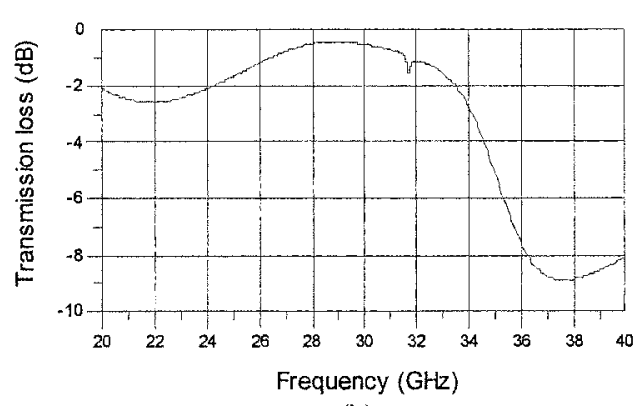

(b)

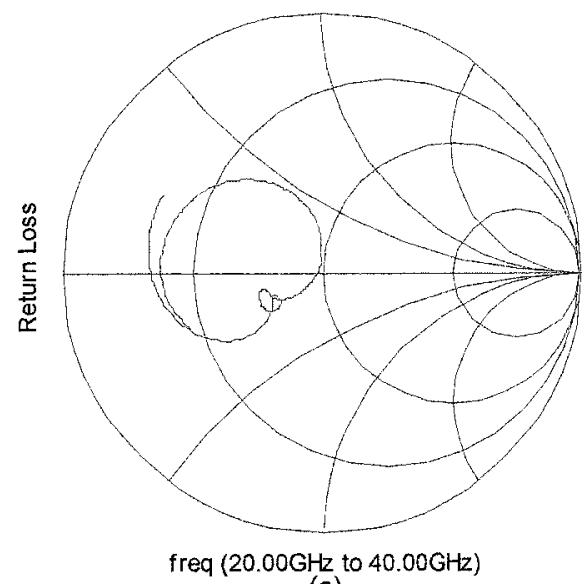

(c)

Figure 3-16: Simulation results for Transition \#2.

From Figure 3-16 results, note that the series stub has added a second resonance point. The two resonance points can be tuned to increase the $-10 \mathrm{~dB}$ bandwidth, however after several attempts the most the bandwidth could be increased was by about $1.5 \mathrm{GHz}$.

\subsubsection{Coupler \#2 design:}

The Transition \#2 design was implemented in a coupler configuration, shown in Figure 317. 


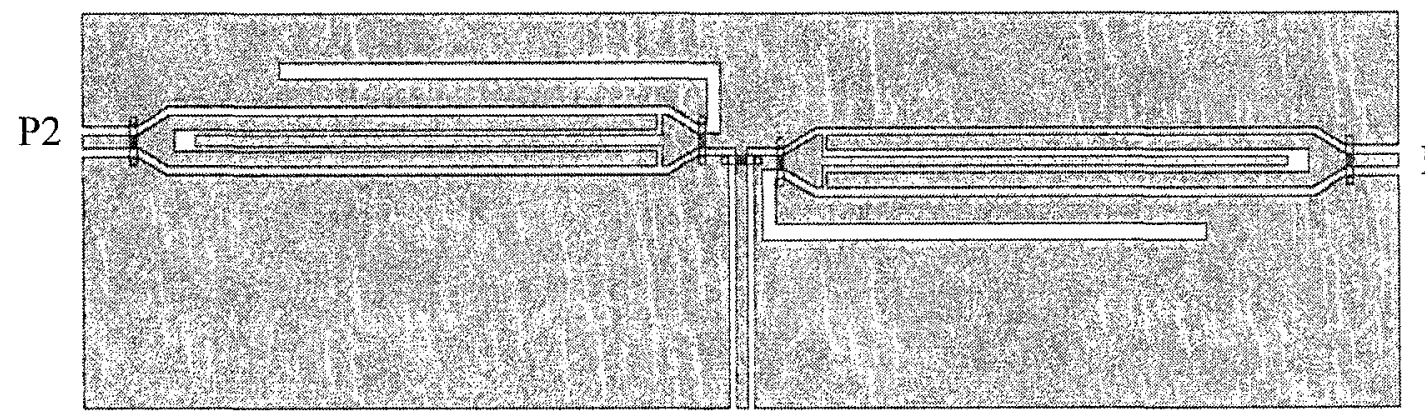

P1

Figure 3-17: Coupler \#2

The results of simulating Coupler \#2 in Momentum are shown below.

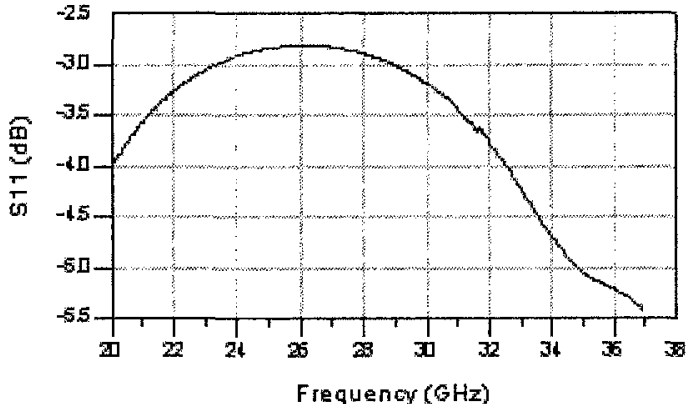

(a)

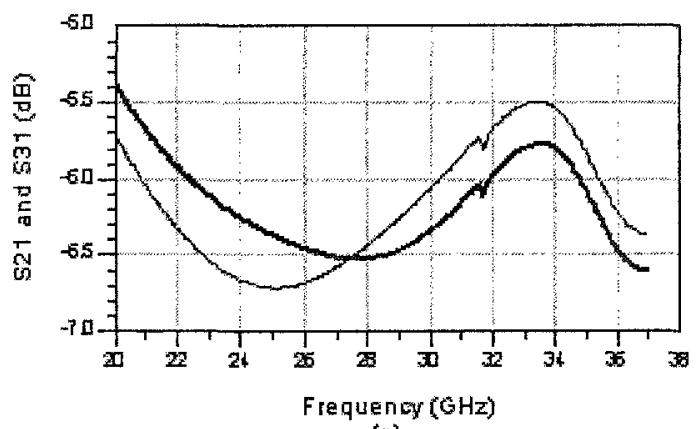

(c)

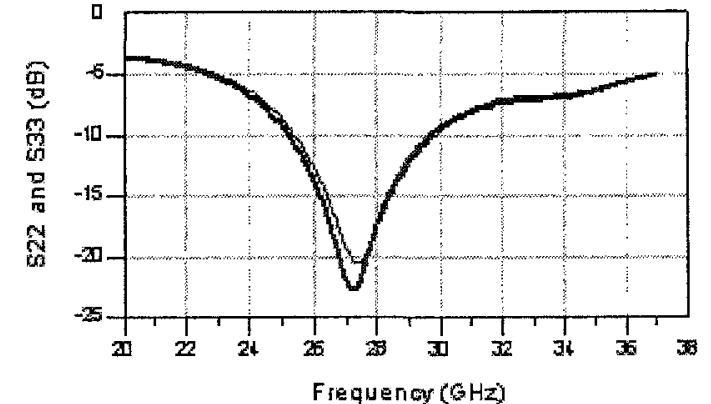

(b)

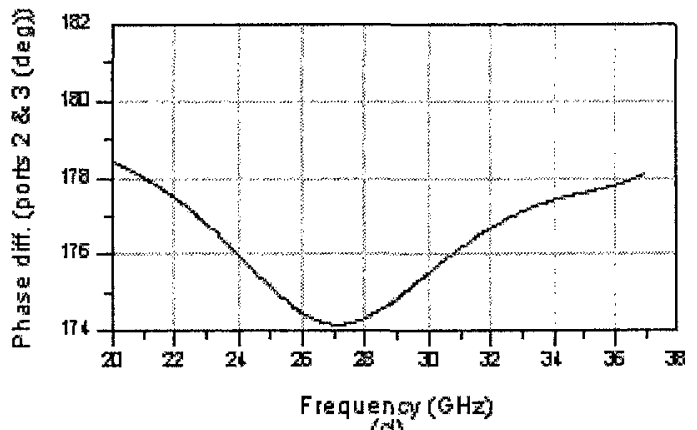

(d)

Legend:

Thin line - Port 2

Thick line - Port 3

Figure 3-18: Simulation results for Coupler \#2.

The Figure 3-18 results show that the reflection coefficients for port 2 and port 3 resemble that of the original transition design. The phase difference between the output 
ports is $+/-2^{\circ}$ (from $178^{\circ}$ to $174^{\circ}$ ). There is no concern at this point with the phase difference not being centered on $180^{\circ}$, as this can be tuned in the final design by increasing the length of one of the output ports of the coupler by a slight amount. More important is maintaining the drifting of the phase difference within $1^{\circ}$ or $2^{\circ}$. Note also the degradation in the reflection coefficient at port 1 . This is due to the fact that it is impossible to match perfectly a loss-less, reciprocal, three-port device unless there is isolation between the two output ports [16].

Since the new coupler can never have all ports matched perfectly, a strategy for achieving the best possible match was derived. For the coupler designs in this thesis the focus was on matching the output ports to $50 \Omega$, while leaving the input port - port 1 - to be matched later using an additional matching circuit. This strategy yields matching circuits for the output ports that are symmetrical, making it easier to find the best possible match for the input of the three-port network. For this reason the unattractive VSWR graphs for port 1 are not a concern in the various coupler designs at the moment, since port 1 is doomed to be mismatched with $50 \Omega$ if the output ports are matched to $50 \Omega$. This must be taken into account when putting together a coupler for use in an actual circuit, as will be seen later in a single balanced mixer.

\subsubsection{Transition \#3 design:}

For the third design iteration, circular slot lines were added to replace the straight slotline stubs. This was done to ensure an effective short at the end of the stubs. Using a circular path splits the signal in two and reunites the signals on the opposite end of the circle in 
opposite phases, which cancels the signal and creates a virtual short, as shown in section 2.4. The virtual short occurs regardless of frequency, making this a more broadband termination for the unused slot. Figure 3-19 of Transition \#3 is given below.

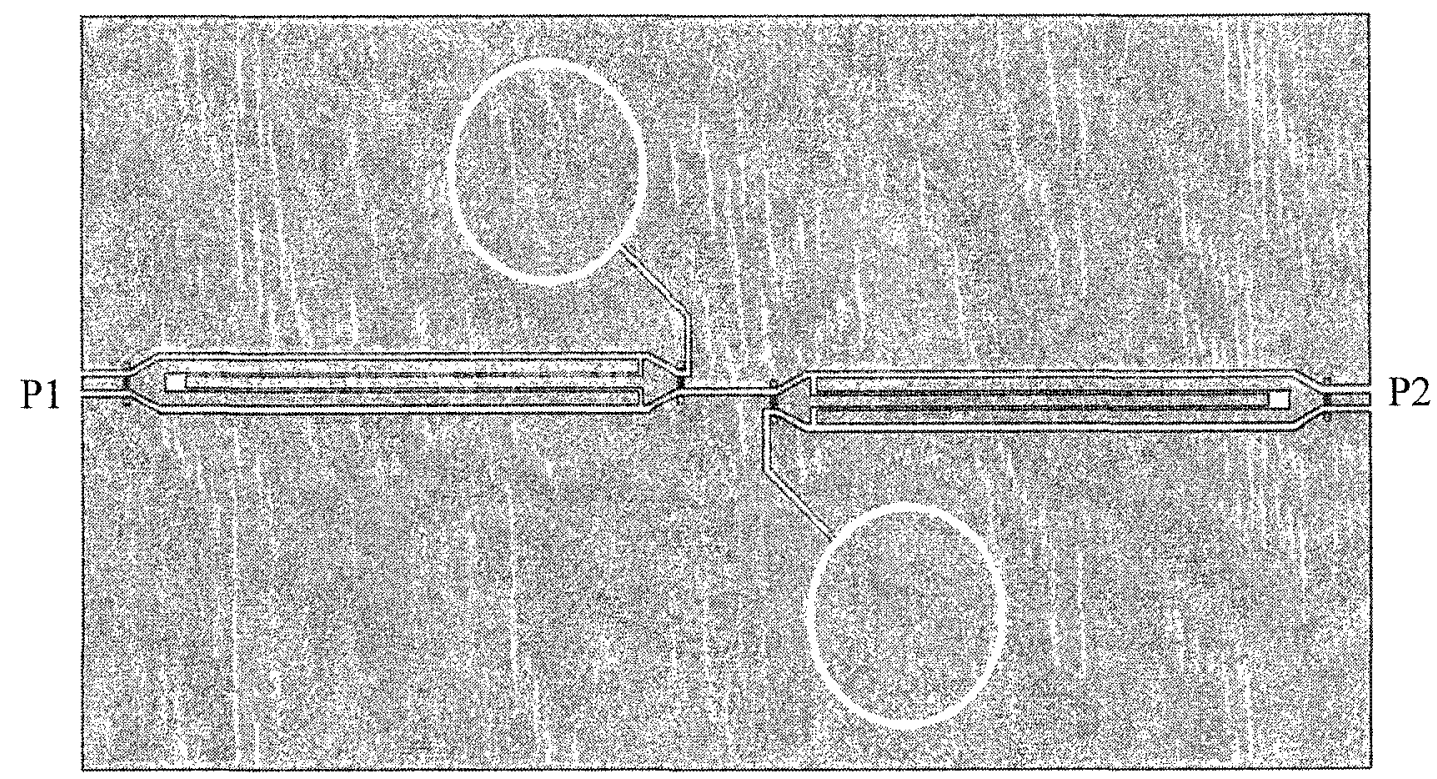

Figure 3-19: Transition \#3

The results of this transition being simulated in Momentum are given in Figure 3-20. 


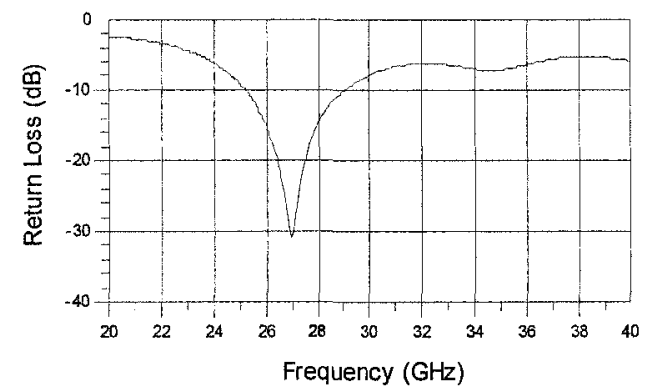

(a)

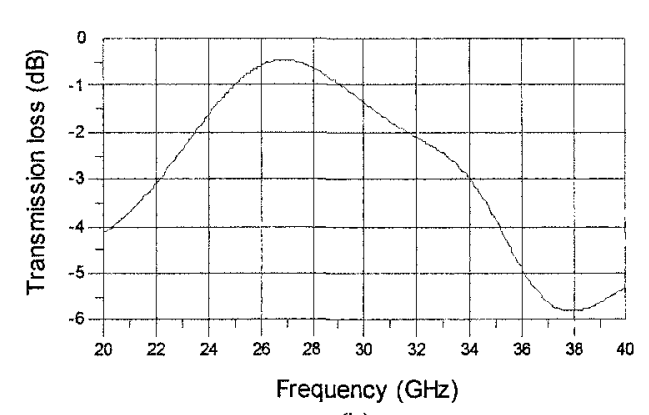

(b)

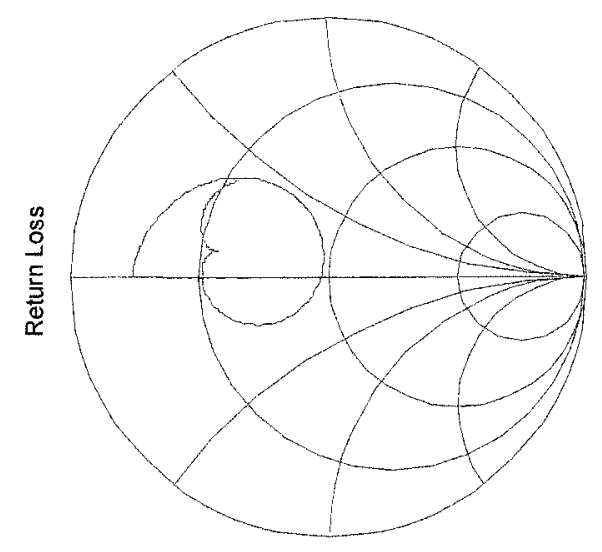

freq $(20.00 \mathrm{GHz}$ to $40.00 \mathrm{GHz})$

(c)

Figure 3-20: Simulation results for Transition \#3.

Figure 3-20(a) shows that Transition \#3 suffers from reduced $-10 \mathrm{~dB}$ bandwidth. This appears discouraging at first, however when implemented in a coupler there is improvement in the phase difference such that the variation is within $+/-1.5^{\circ}$. There is therefore a tradeoff between return loss bandwidth and better phase response for this design. 


\subsubsection{Coupler \#3 design:}

The figure of Coupler \#3 along with the data are given below in Figures 3-21 and 3-22.

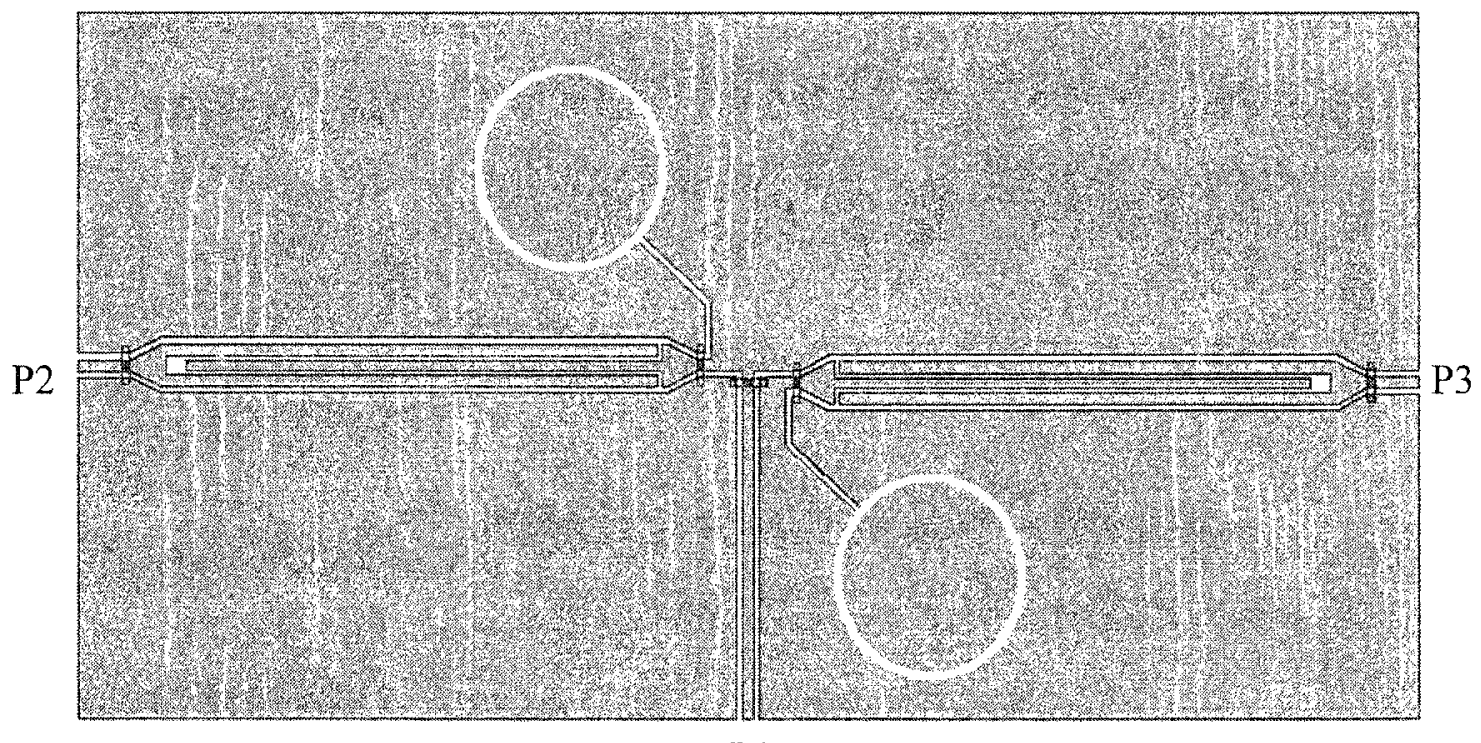

P1

Figure 3-21: Coupler \#3

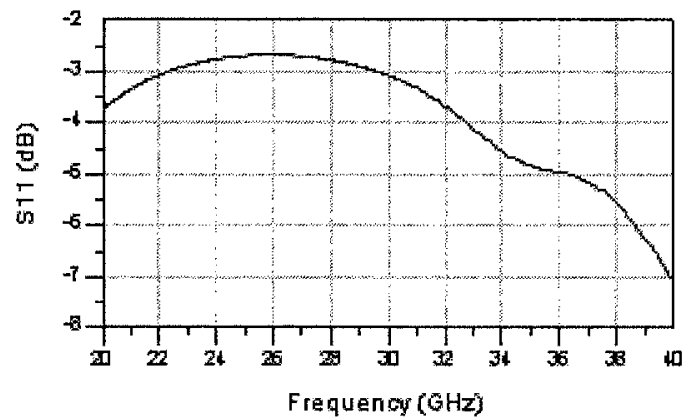

(a)

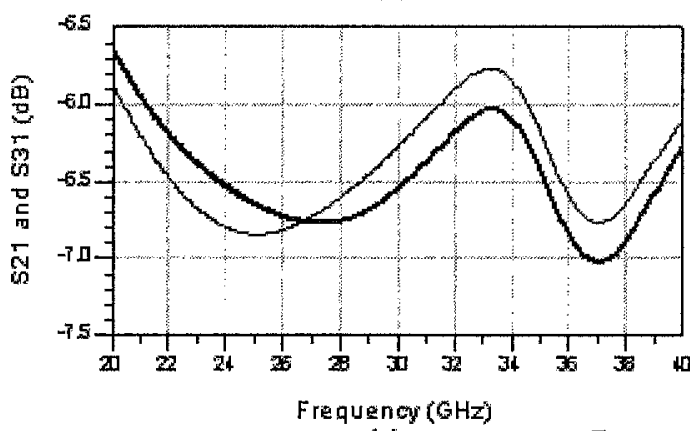

(c)

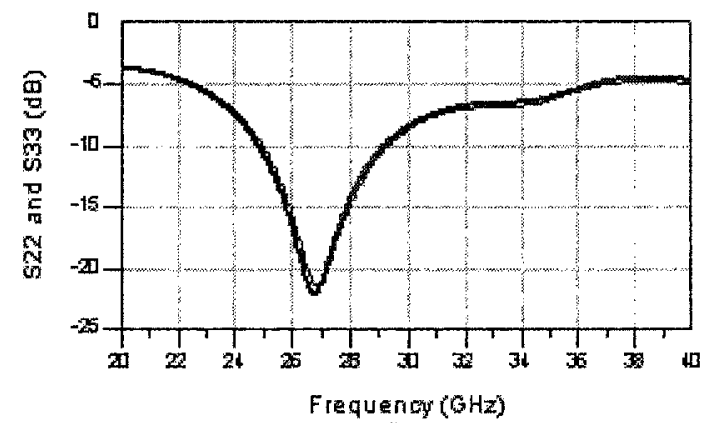

(b)

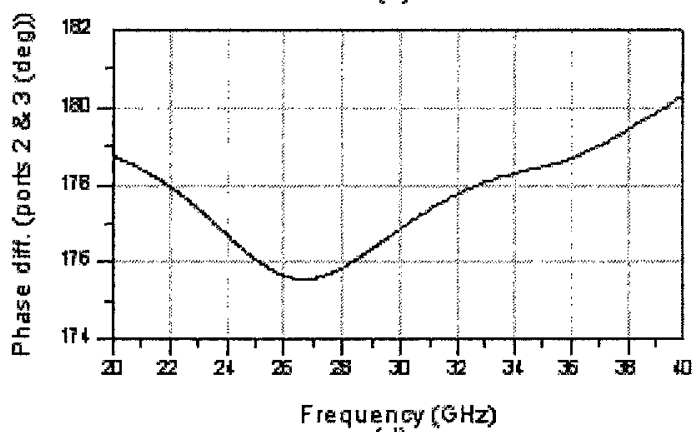

(d)

Legend:

Thin line - Port 2

Thick line - Port 3

Figure 3-22: Simulation results for Coupler \#3. 
Up to this point, the coupler designs have achieved a phase difference that varies no more than $+/-1.5^{\circ}$ from $20 \mathrm{GHz}$ to $40 \mathrm{GHz}$, however the bandwidth in terms of reflection coefficient at the output ports has not, so far, been greater than $6 \mathrm{GHz}$.

\subsubsection{Transition \#4 design:}

For the transition \#4 design, the focus turns to minimizing the impedance of the series resonant structure. One technique for creating a lower apparent impedance is by placing two identical stubs in parallel, effectively halving the impedance. This is illustrated in the equivalent circuit diagram shown in Figure 3-23.

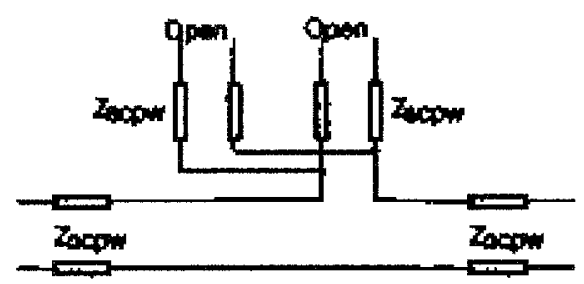

Figure 3-23: Equivalent circuit of two stubs in parallel [15].

The above technique was implemented in a transition design, shown in Figure 3-24 below: 


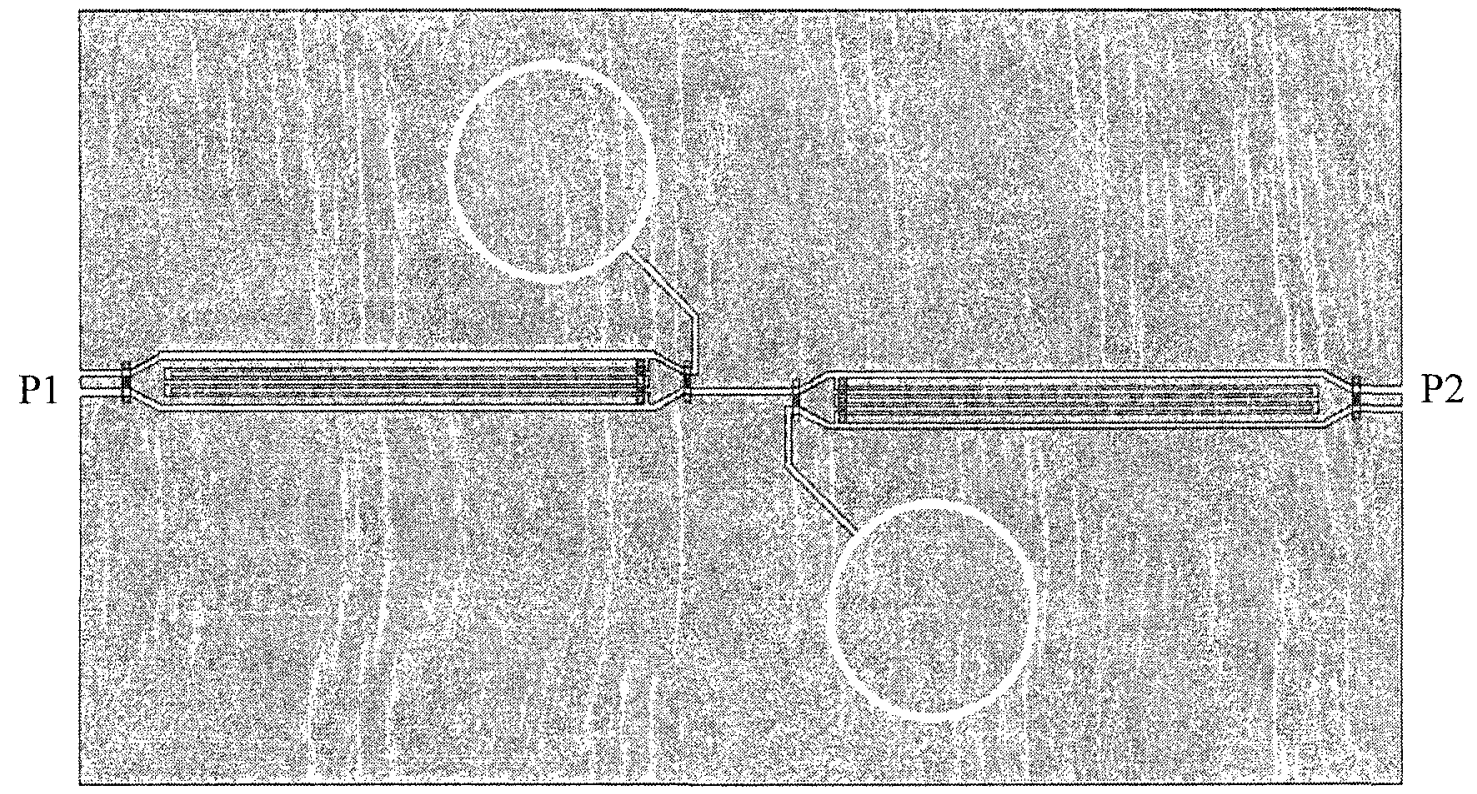

Figure 3-24: Transition \#4

The length of each of the series stubs is $930 \mu \mathrm{m}$. The data for Transition \#4 is given in Figure 3-25. 


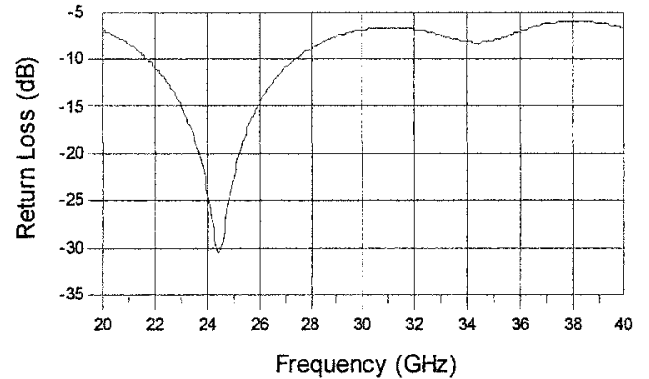

(a)

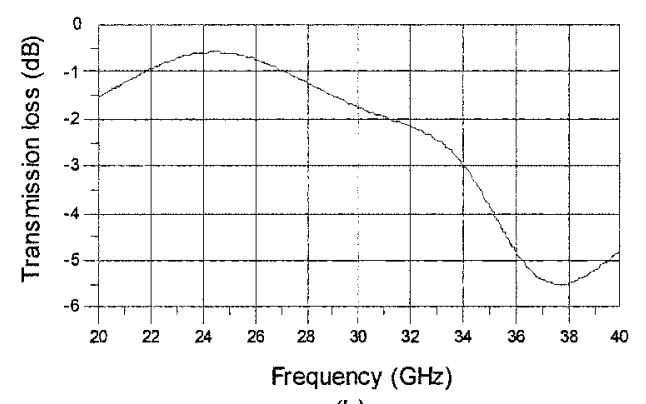

(b)

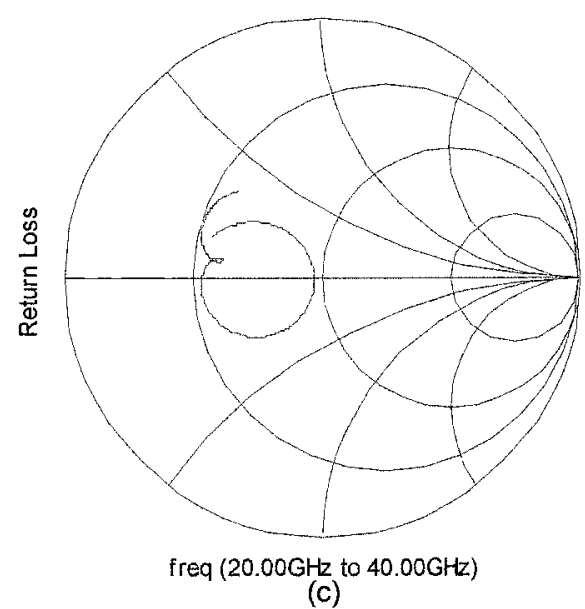

Figure 3-25: Simulation results for Transition \#4.

The data in Figure 3-25 shows no appreciable improvement in the reflection coefficient over the 20 to $40 \mathrm{GHz}$ band. It is probable that because it was necessary to make the two series stubs so narrow, and thus make the impedance of the stubs greater, then taking the two stubs in parallel produced a combined impedance that was not much less than the impedance with a single series stub. The CPW slots for the series stubs were made as small as possible to reduce the characteristic impedance of each stub, however physical limitations of the fabrication process prevented further design improvement. 


\subsubsection{Coupler \#4 design:}

The Transition \#4 design was implemented as a coupler, shown in Figure 3-26.

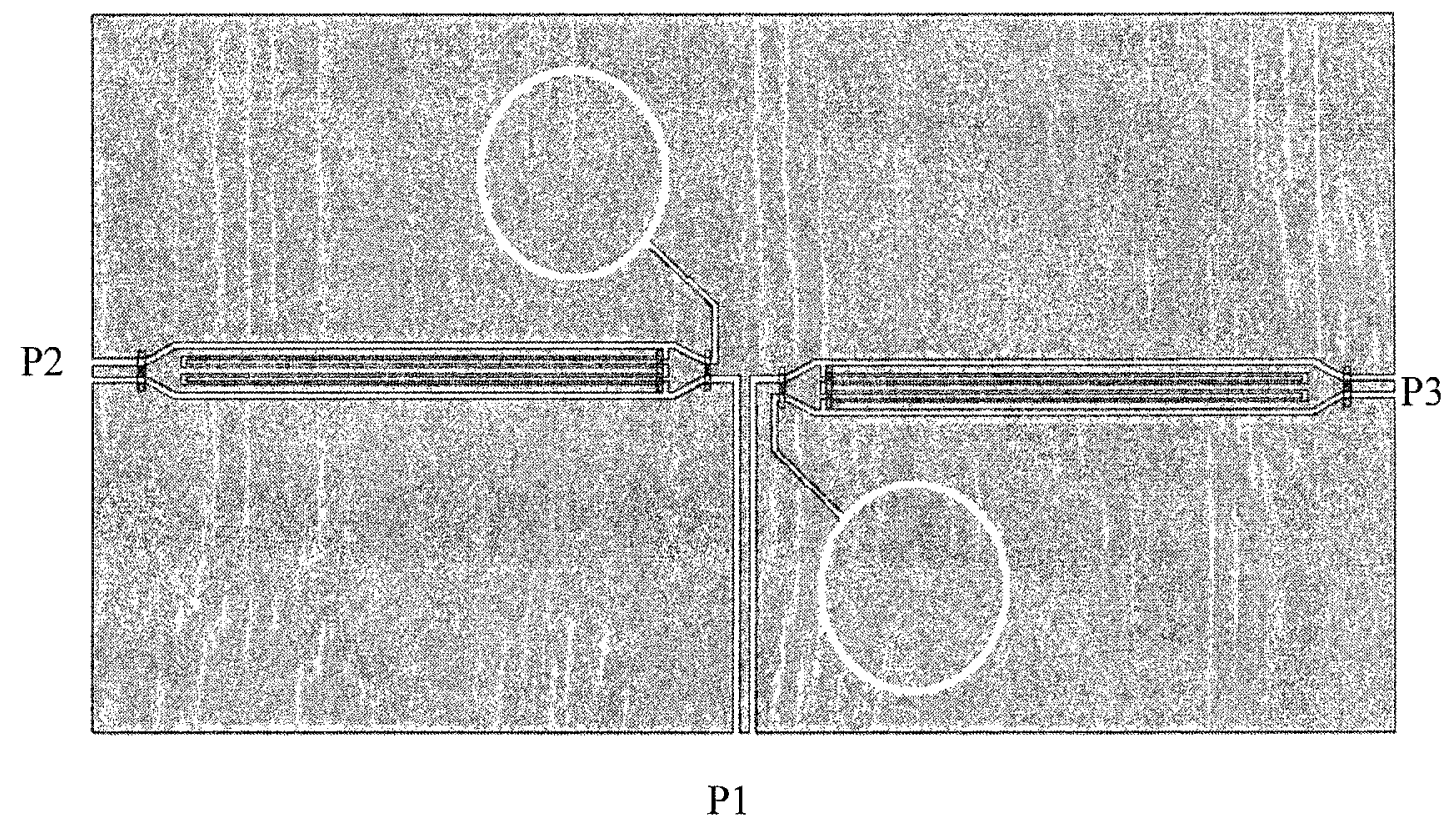

Figure 3-26: Coupler \#4

Data for Coupler \#4 is given in Figure 3-27. 


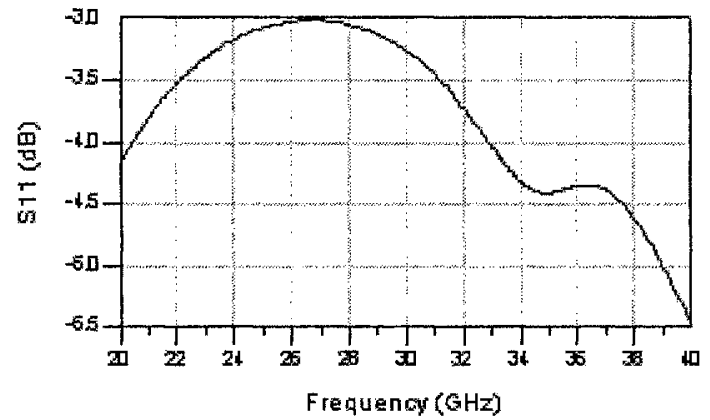

(a)

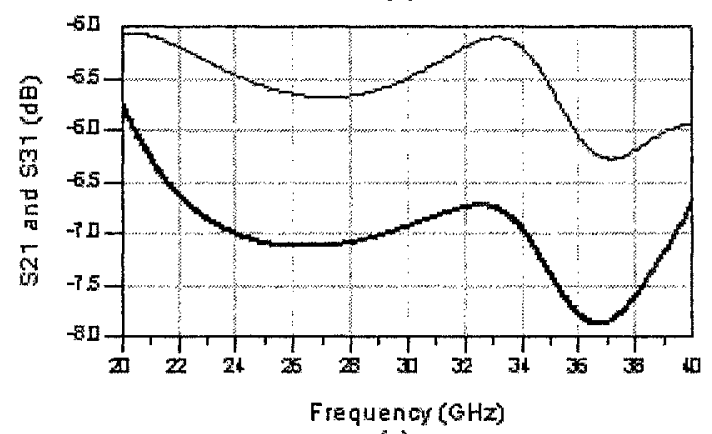

(c)

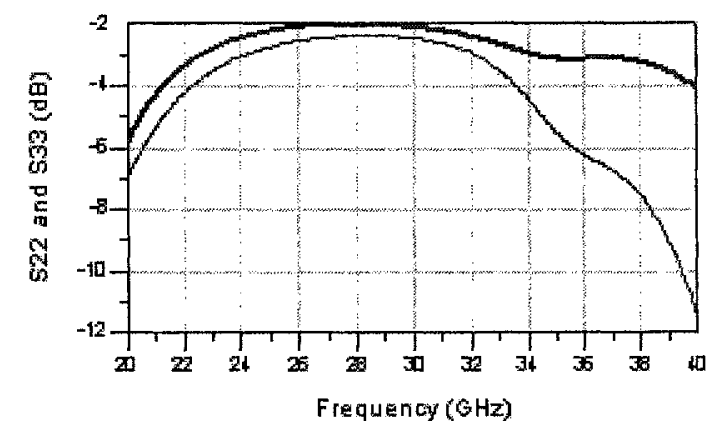

(b)

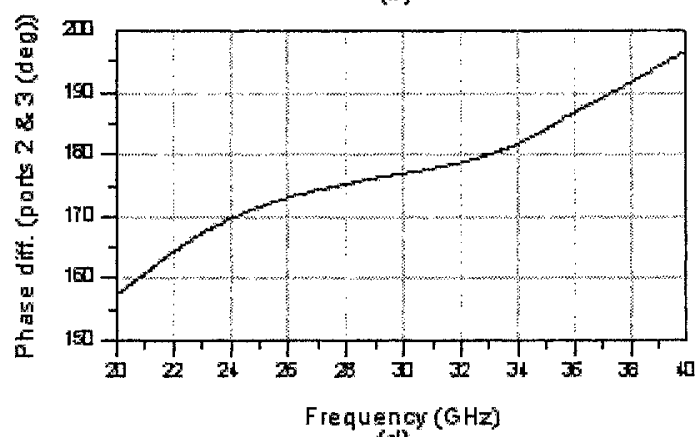

(d)

Thin line - Port 2

Thick line - Port 3

Figure 3-27: Simulation results for Coupler \#4.

Figure 3-27 shows that Coupler \#4 performs poorly in simulation. It is noted also that the performance is worse than predicted by the transition design alone. It is likely that the combination of the two stubs creates an impedance mismatch that is exacerbated by the transfer characteristics of the three-port T-junction. There is a clear difference between the results for Port 2 and for Port 3, which is difficult to explain, as the two sides of the coupler are symmetric. The only difference between either ports is the direction of the field entering the series stubs. It is therefore likely that the parallel series stubs have different impedance characteristics based on the orientation of the field. 


\subsection{Final Transition and Coupler Design}

\subsubsection{Final Transition Design:}

For the fifth and final transition design, a novel approach of combining a series microstrip stub coupled to the coplanar circuit was proposed. The idea stems from the fact that if a microstrip line could be produced using the normal metallization layer [IN] and another metal layer below it [BE], the characteristic impedance of the series stub could be made very low since 1) the layers are so close together $-0.950 \mu \mathrm{m}$, and 2) the width of the microstrip line could be increased. Both of these variables help to increase the distributed capacitance of the line, which in turn lowers the characteristic impedance of the line, since $Z_{o}=\sqrt{\frac{L}{C}}$ where $\mathrm{L}$ and $\mathrm{C}$ are the distributed inductance and capacitance of the line.

The two metallization layers chosen were the [IN] layer (top) and the [BE] layer (bottom) which are separated by $800 \mathrm{~nm}$ of $\mathrm{S}_{\mathrm{i}} \mathrm{O}_{2}\left(\varepsilon_{\mathrm{r}}=5\right)$ and $150 \mathrm{~nm}$ of $\mathrm{Si}_{3} \mathrm{~N}_{4}\left(\varepsilon_{\mathrm{r}}=6.8\right)$. The optimal length and width of the microstrip stub to set the resonance point to $30 \mathrm{GHz}$ was found theoretically and tuned through simulation. The Figure 3-28 shows the optimized final transition. 


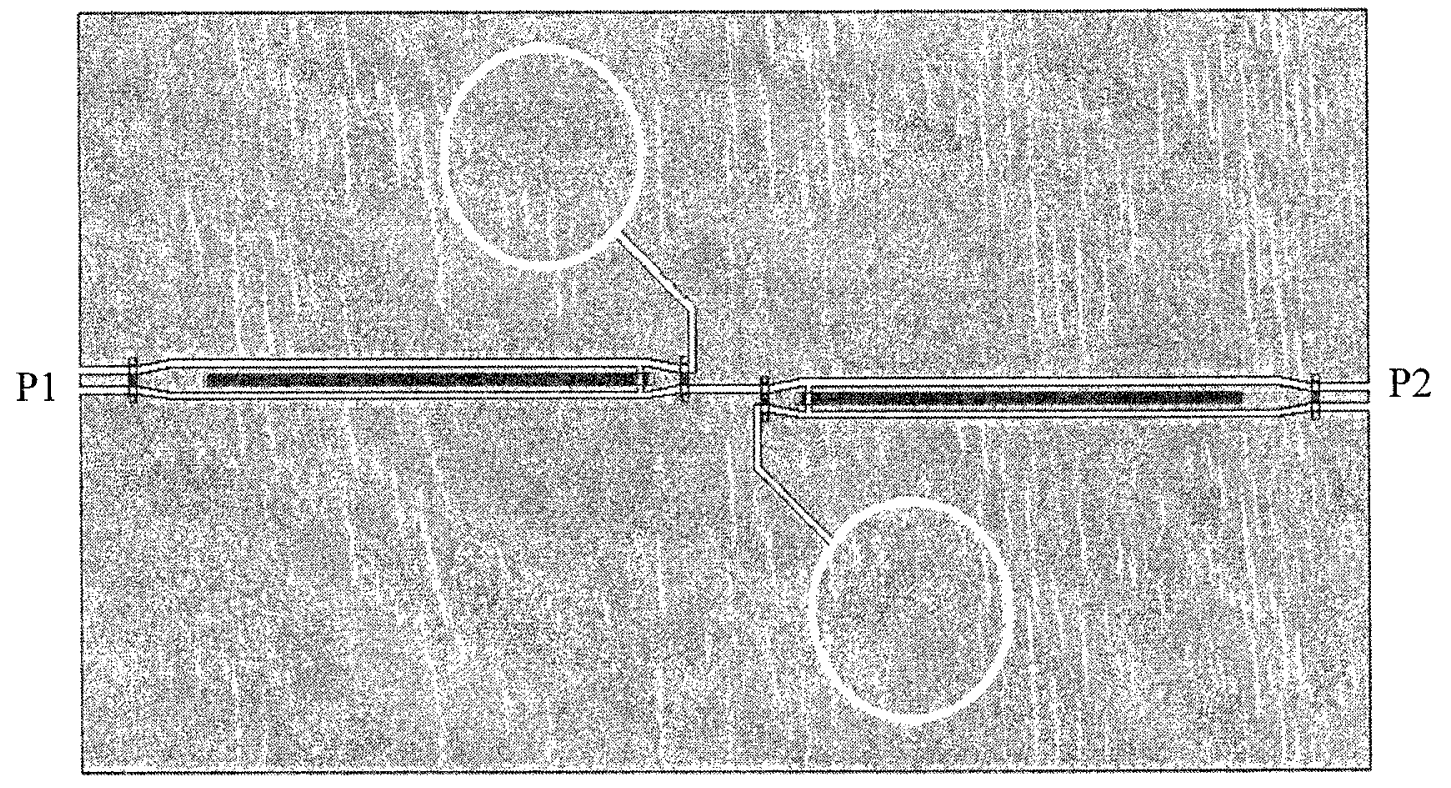

Figure 3-28: Final Transition

The length of the series microstrip stub in the above figure is $800 \mu \mathrm{m}$, while its width is $\mathrm{w}=20 \mu \mathrm{m}$. The simulation data for the final transition is given in Figure 3-29 for 20 $\mathrm{GHz}$ to $40 \mathrm{GHz}$. 


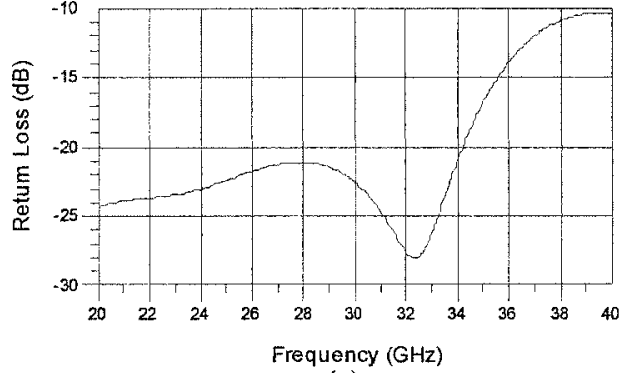

(a)

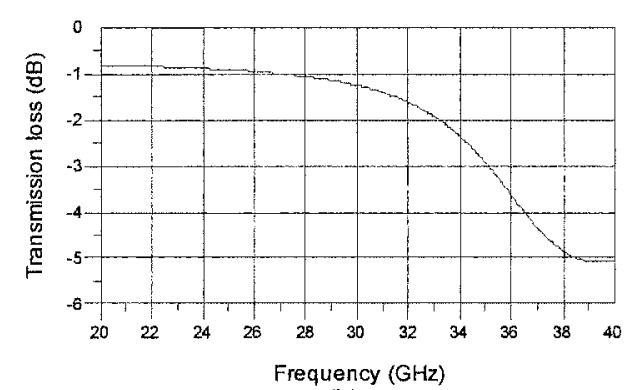

(b)

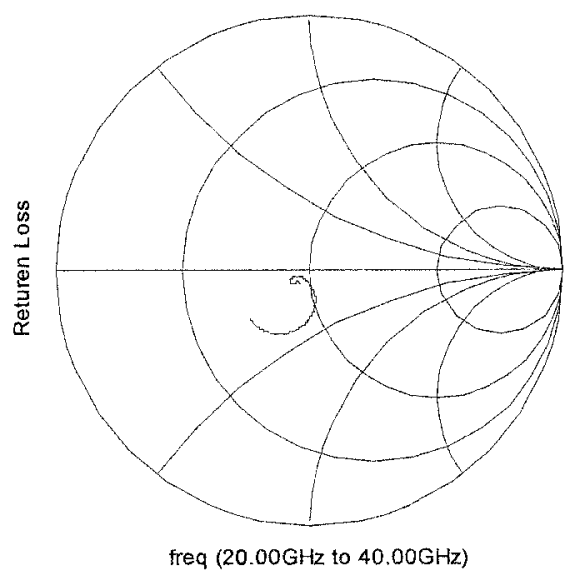

(c)

Figure 3-29: Simulation results for Final Transition.

The most notable observation in the data of Figure 3-29 is the dramatic improvement in bandwidth over previous transition designs. The $-10 \mathrm{~dB}$ bandwidth extends from $20 \mathrm{GHz}$ to $40 \mathrm{GHz}$ and appears to continue below $20 \mathrm{GHz}$. This represents a bandwidth for the transition of at least $20 \mathrm{GHz}$, or $50 \%$.

To explain the improvement in performance, standard equations for calculating $Z_{0}$ of a microstrip line are found in $[10, p .162]$. In [10] there are actually two equations - one for when $w / d \geq 1$, and one for $w / d \leq 1$, where $w / d$ is the ratio of strip width to substrate thickness. 
For the series microstrip stub in the final transition:

$\frac{w}{d}=\frac{20 \mu m}{0.950 \mu m}=21.053$, Which is $>1$ and therefore the equation for $Z_{o}$ is as follows [10]:

$$
Z_{o}=\frac{120 \pi}{\sqrt{\varepsilon_{e}\left[\frac{w}{d}+1.393+0.677 \ln \left(\frac{w}{d}+1.444\right)\right]}}
$$

Where $\varepsilon_{\mathrm{e}}$ is the effective dielectric constant of a microstrip line, and is defined as [10]

$$
\varepsilon_{e}=\frac{\varepsilon_{r}+1}{2}+\frac{\varepsilon_{r}-1}{2} \frac{1}{\sqrt{1+\frac{12 d}{w}}}
$$

Where: $-d / w=(21.053)^{\wedge}-1=0.0475$

- $\varepsilon_{\mathrm{r}}$ is the relative permittivity of the dielectric material between the strip and the ground plane - in this case the strip [Be layer] is actually below the ground plane [IN layer], so the microstrip line is actually inverted. The value of $\varepsilon_{r}$ is not a straightforward quantity for the OMMIC ${ }^{\mathrm{TM}}$ layers, since there are actually two dielectric layers between the $[\mathrm{IN}]$ and $[\mathrm{BE}]$ layers. In this case, $\varepsilon_{\mathrm{r}}$ was taken to be the weighted average of the $\varepsilon_{\mathrm{r}}$ 's for each dielectric, as follows:

For $\mathrm{S}_{\mathrm{i}} \mathrm{O}_{2}\left(\varepsilon_{\mathrm{r}}=5\right)$ and $\mathrm{S}_{\mathrm{i}} \mathrm{N}_{4}\left(\varepsilon_{\mathrm{r}}=6.8\right)$

$$
\varepsilon_{r}=\left(\frac{800 \mathrm{~nm}}{800 \mathrm{~nm}+150 \mathrm{~nm}}\right)(5)+\frac{150 \mathrm{~nm}}{800 \mathrm{~nm}+150 \mathrm{~nm}}(6.8)=5.3
$$

Therefore the effective dielectric constant, $\varepsilon_{\mathrm{e}}$, is estimated to be,

$$
\varepsilon_{e}=\frac{5.3+1}{2}+\frac{5.3-1}{2} \frac{1}{\sqrt{1+\frac{12 d}{w}(0.0475)}}=4.866
$$

Finally, $Z_{0}$ is calculated as: 
$Z_{o}=\frac{120 \pi}{\sqrt{4.866}[21.053+1.393+0.667 \ln (21.053+1.444)]}=6.97 \Omega \approx 7 \Omega$

The above result shows that indeed the series stub has very low impedance. As a check, the stub can be view as being a parallel plate waveguide rather than a microstrip line since the ground plane is really just the narrow patch of [IN] layer in the center of the CPW waveguide. The characteristic impedance for a parallel plate waveguide is $[10$, p.113].

$Z_{o}=\frac{\eta d}{w}$

Where $\eta=\sqrt{\frac{\mu}{\varepsilon}}=\sqrt{\frac{\mu_{o}}{(5.3) \varepsilon_{o}}}=163.64$

$$
\begin{aligned}
& \mathrm{d}=0.950 \mu \mathrm{m} \\
& \mathrm{w}=20 \mu \mathrm{m}
\end{aligned}
$$

Therefore,

$Z_{o}=(163.64)\left(\frac{0.95}{20}\right)=7.773 \Omega$

This result is reasonably close to the one found using the equation for microstrip lines, so it can be reasonably concluded that the real $Z_{0}$ would lie somewhere between $7 \Omega$ and $8 \Omega$.

Such a low series stub impedance, has effectively achieved close to the ideal scenario described in previous theory, meaning that creating a series stub as close to $0 \Omega$ as possible extended the bandwidth over which the stub helps to eliminate the complex terms in the equation for $Z_{s}$, as predicted by equation $3-5$. 
To illustrate the effect of the series microstrip stub, the final transition was re-simulated without the series stub. This new transition was named transition \#6 and is illustrated in Figure 3-30.

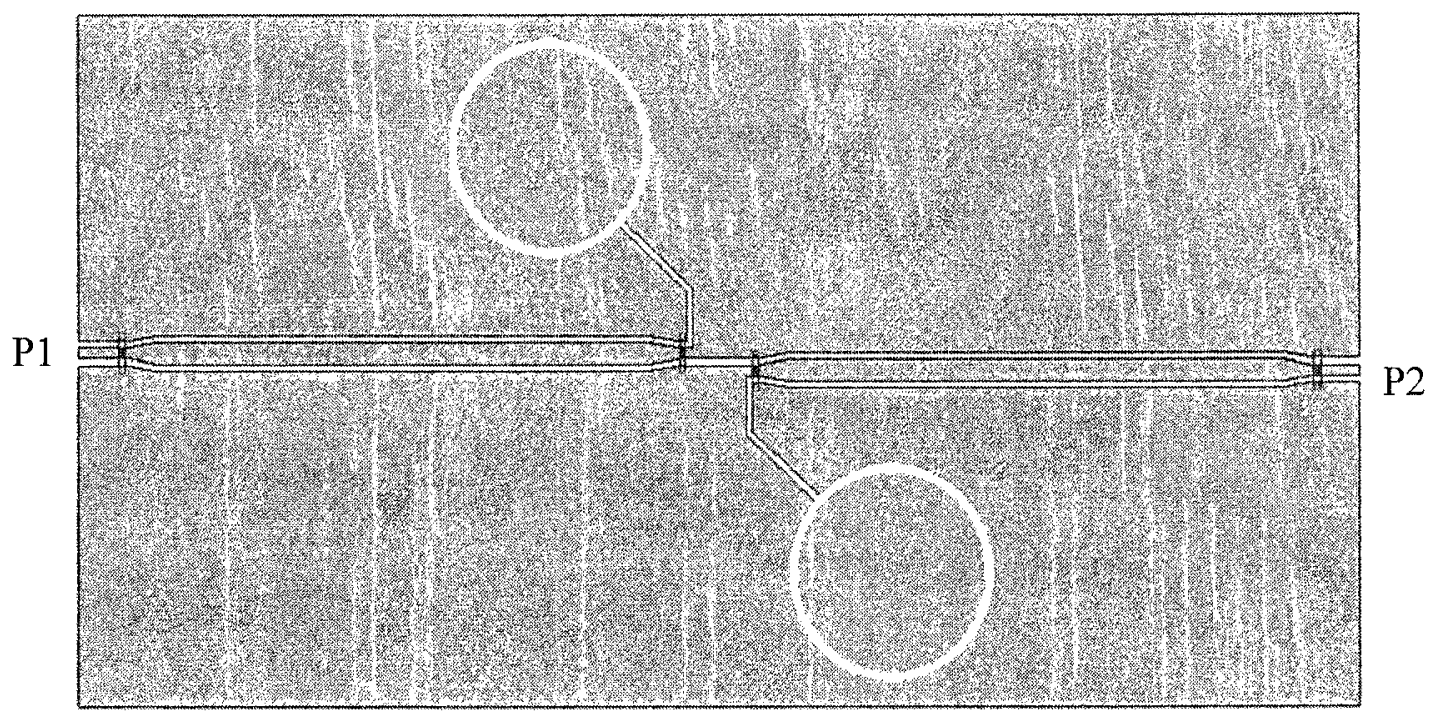

Figure 3-30: Final transition without series microstrip stub - transition \#6.

The results for the simulation of transition \#6 are in Figure 3-31. 


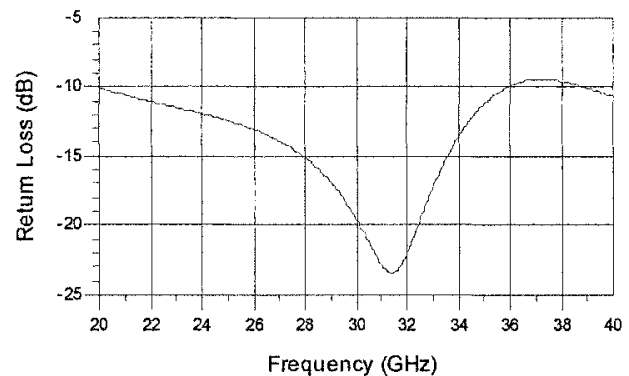

(a)

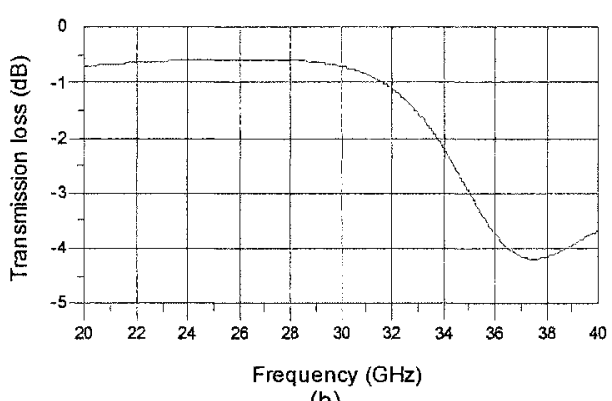

(b)

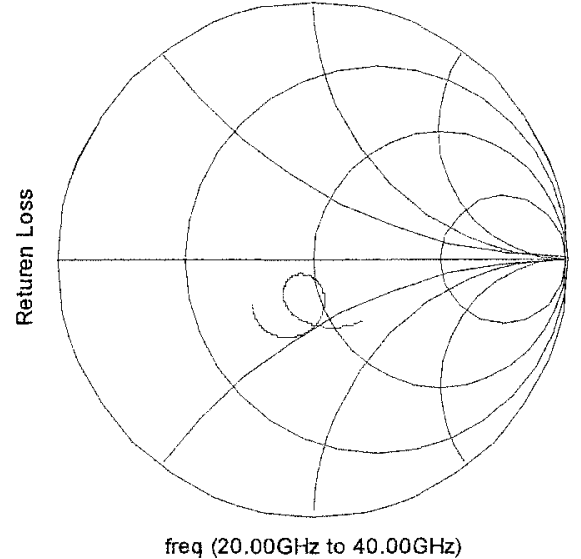

(c)

Figure 3-31: Simulation results for Transition \#6.

The above results clearly show the degradation in the reflection coefficient below the main resonant point at $31.5 \mathrm{GHz}$, as compared to the transition with the series microstrip stub. This strongly supports the theory that explained how the series stub increases bandwidth in the transition design. 


\subsubsection{Final Coupler Design:}

The performance of the final transition design is impressive, but will the same hold for the coupler design using the same methods? Recall that because the coupler is a nonreciprocal, lossless, three-port device with no isolation between the ports, it is expected that the match of the output ports to deteriorate slightly from the original transition design. Figure 3-32 shows the final coupler design.

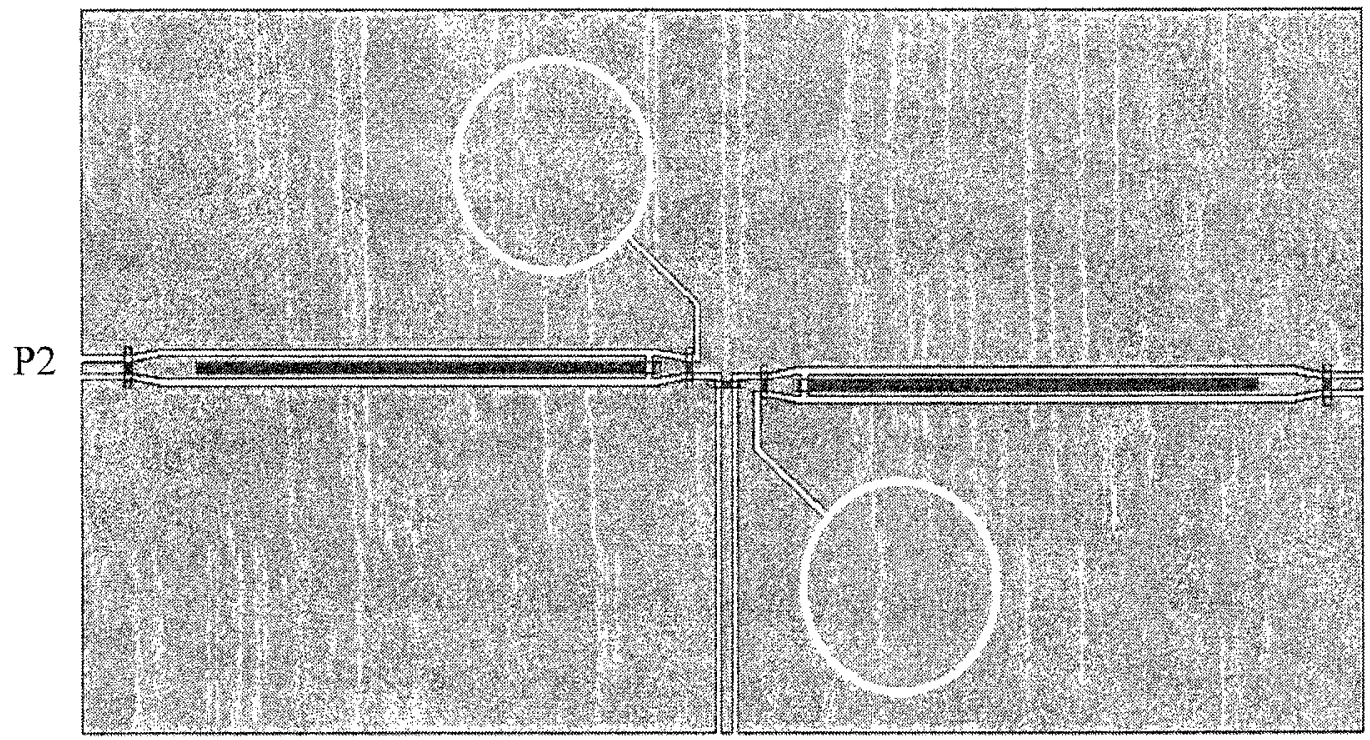

P1

Figure 3-32: Final Coupler 
The data for the final coupler is given in Figure 3-33.

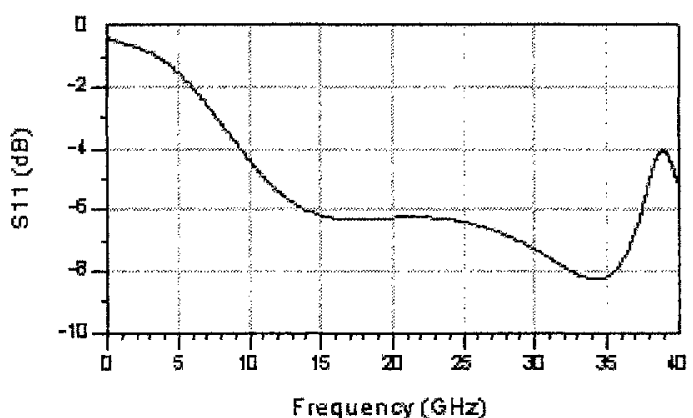

(a)

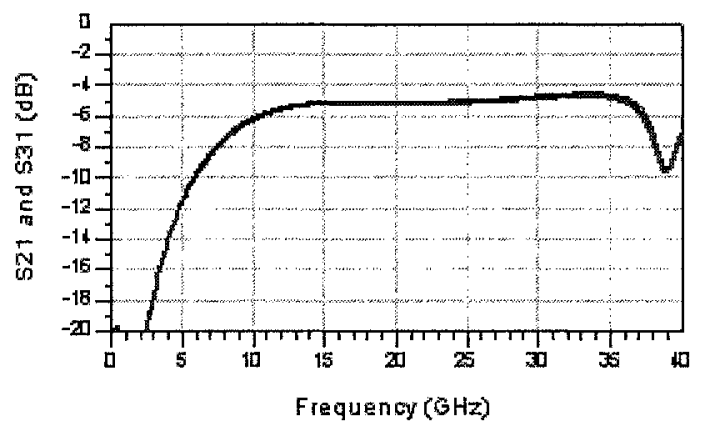

(c)

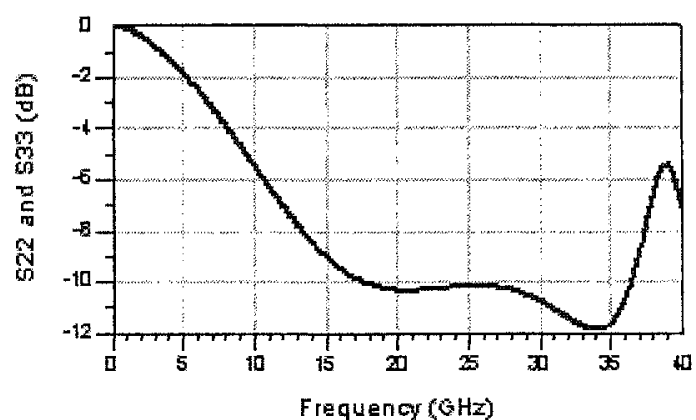

(b)

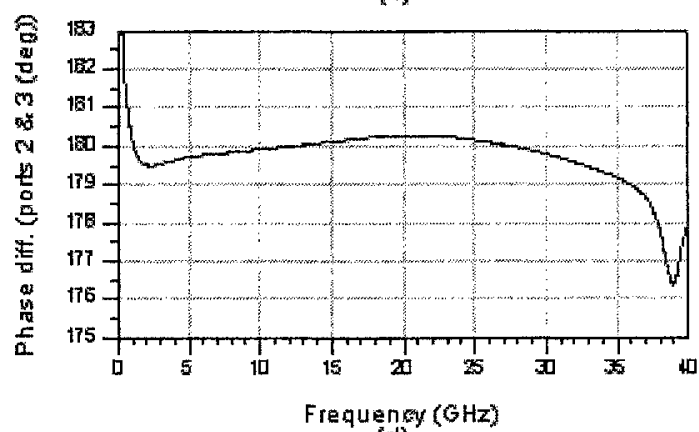

(d)

Thin line - Port 2

Thick line - Port 3

Figure 3-33: Simulation results for Final Coupler.

The final coupler was simulated from 0 to $40 \mathrm{GHz}$ to show where the lower cutoff frequency lies. In the results of Figure 3-33 note some degradation in the return losses at the output, as compared to the transition design, due to lack of isolation between the output ports, however the performance is still substantially better than all previous iterations. The $-10 \mathrm{~dB}$ bandwidth for this fifth and final coupler design is $17 \mathrm{GHz}$, or $42 \%$, which puts this coupler in contention with other broadband planar couplers. There are several distinct advantages that the new coupler has over its predecessors, which are listed below and will be highlighted in the next chapter where the coupler is integrated in a single-balanced mixer. 
One last note about the final coupler is the very flat curve of the phase difference between port 2 and port 3. The change in the phase difference is now less than $1^{\circ}$ over the 17 $\mathrm{GHz}$, which is excellent. This should guarantee good isolation between input signals over the overall bandwidth, as will be demonstrated in the mixer design in the next chapter.

The following list summarizes the advantages of the new coupler:

- Easy to design

- Provides a $180^{\circ}$ phase split: Better for mixer applications for reasons discussed in $[11$, p.269].

- Broadband: Return loss bandwidth is quite large, while the phase balance beats all other $180^{\circ}$ couplers.

- Can be an efficient user of space: Independent output paths mean the coupler can be put anywhere in the layout. This allows a measure of flexibility, which aids efficient layout. Also, with only a small penalty in performance, the ring slotline stubs can be replaced with regular slotline stubs that follow the outputs of the T-junction, which would make the circuit even more compact. 
The new coupler is compared below in Table 3-1 with the ideal couplers discussed in chapter 2 .

Table 3-1: Comparison of simulated performance of new coupler with ideal couplers in the $20 \mathrm{GHz}$ to 40 GHz band.

\begin{tabular}{|c|c|c|}
\hline & $\begin{array}{l}\text { Amplitude } \\
\text { Response BW }\end{array}$ & $\begin{array}{c}\text { Phase } \\
\text { Response BW }\end{array}$ \\
\hline $\begin{array}{l}\text { Branchline } \\
\text { Coupler }\end{array}$ & $8 \mathrm{GHz}$ & $10 \mathrm{GHz}$ \\
\hline $\begin{array}{l}\text { Hybrid-Ring } \\
\text { Coupler }\end{array}$ & $12 \mathrm{GHz}$ & $5 \mathrm{GHz}$ \\
\hline $\begin{array}{l}\text { Coupled Line } \\
\text { Coupler }\end{array}$ & $14 \mathrm{GHz}$ & (* Large) \\
\hline New Coupler & $17 \mathrm{GHz}$ & (*Large) \\
\hline
\end{tabular}

${ }^{*}$ Large in this case means covering at least $80 \%$ of the bandwidth.

Table 3-1 shows that the simulated results for the new coupler are a significant improvement to the results of the other couplers. Compared to the Hybrid-Ring, the only other coupler that provides a $180^{\circ}$ phase split, the new coupler improves return loss bandwidth by $41 \%$. The new coupler also improves the phase balance bandwidth by $800 \%$, when compared to the Hybrid-Ring coupler.

The next 2 chapters describe how the new coupler could be used in a single-balanced mixer, as well as providing experimental validation of the design from a real fabricated coupler and mixer. 


\section{CHAPTER 4: NEW COUPLER USED IN BASIC MIXER}

In the previous chapter, the design of a novel coupler incorporating coplanar structures, slotline structures, and microstrip structures was discussed. However the true litmus test of a new coupler would be to demonstrate how the coupler might be used in a real circuit. Therefore this chapter describes the design of a single-balanced, down-converting, diode mixer using the new coupler. There are three types of mixers: 1) single-device mixers, 2) singly-balanced mixers, and 3) doubly balanced mixers [11]. The reason for choosing to design a single balanced mixer is it would require a coupler, which is essential and negates the design of a single-device mixer, but is less complex than a doubly-balanced mixer.

\subsection{Mixer topologies using various types of couplers}

Before introducing the new coupler in a mixer, it is instructive to look at some other types of couplers as they are used in mixers. The following paragraphs discuss different topologies of mixers using some other common couplers. 


\subsubsection{Rat-Race or Ring-Hybrid:}

The basic topology of a single-balanced mixer using a hybrid-ring coupler, or rat-race is given in Figure 4-1.

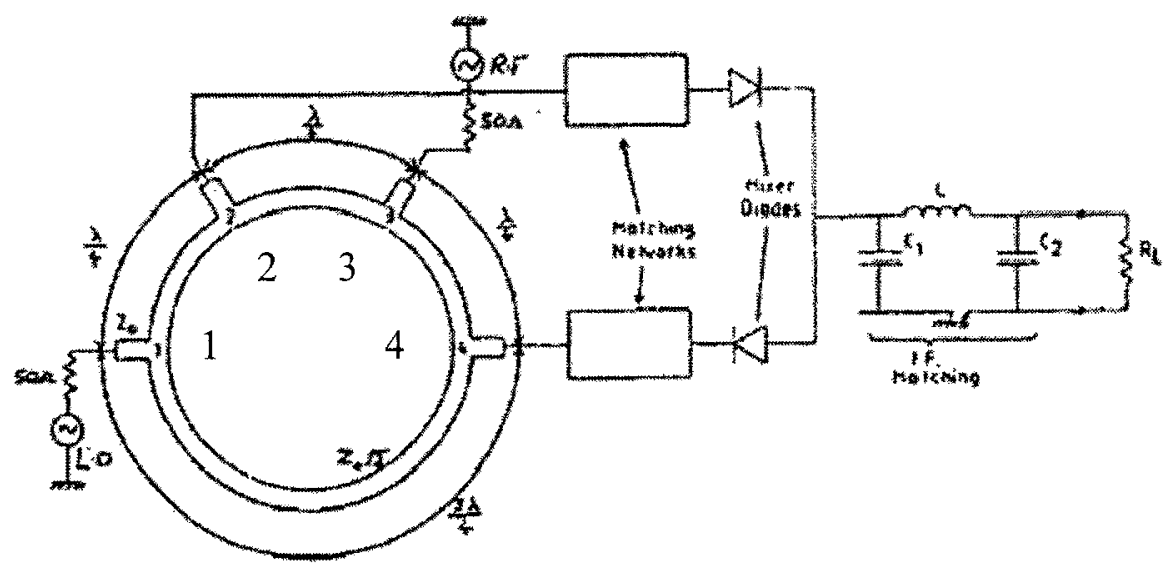

Figure 4-1: Rat-Race mixer topology [17].

In Figure 4-1, we see that the LO input signal enters port 1 and is split between ports 2

and 4 . Since there is a $0.5 \lambda$ difference in the length from port 1 between the two ports, the LO signal will be split into two output signals with an $180^{\circ}$ phase shift. The RF input signal enters port 3 and is split evenly with no phase shift between ports 2 and 4 . As discussed in chapter 2 , it is a property of the rat-race hybrid that the input signal at any port will be split between two ports, while the fourth port is isolated. Thus the RF and LO ports are effectively isolated from one another. Up to $-43 \mathrm{~dB}$ of isolation between the RF and LO ports was achieved in [17]. To extract the IF signal, a circuit serving both as a matching circuit at the IF frequency and as a filter to isolate the output from the RF and LO signal leakage (especially the LO signal, which has much higher power) was used. LO-IF isolation of $-20 \mathrm{~dB}$ was achieved using this circuit [17]. 
The advantages of the hybrid-ring mixer include ease of design, as the hybrid-ring is a well-proven circuit element used quite commonly in mixers. High isolation between the LO and RF ports, which translates into better balance and conversion gain by making the mixing more efficient, is another advantage. Disadvantages of using the ring-hybrid include its narrowband nature. Frequency dependence of the dimensions can make size a problem at lower frequencies. Another disadvantage is that the ring-hybrid is more difficult to implement using coplanar waveguides, though it can be done using finiteground-plane coplanar techniques [18]. Because of the frequency dependence of the structure, the hybrid-ring is typically not considered to be a broadband coupler, however techniques like those given in [18] that use a phase inverter can make the hybrid-ring broadband.

\subsubsection{Branchline coupler:}

The branchline coupler is a quadrature coupler, meaning the phase difference between the output ports is $90^{\circ}$. A Figure of this coupler is given below.

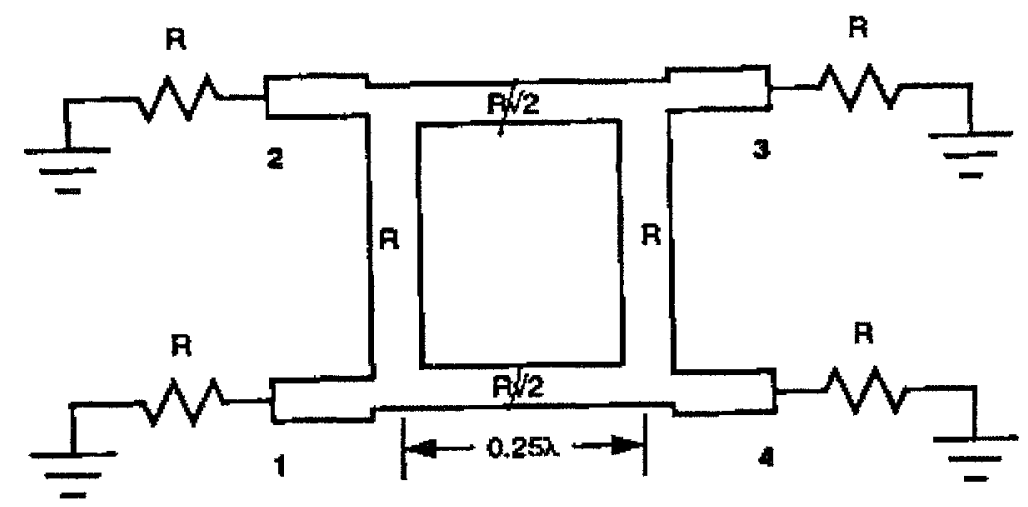

Figure 7.14 Single-section brancti-line bybrid.

Figure 4-2: Branchline Coupler [11, p.253]. 
The single-balanced mixer topology using a branchline coupler found in most papers -i.e. [19], [20], and [21] - is essentially the textbook topology of a balanced mixer using an ideal quadrature coupler, shown in the Figure below.

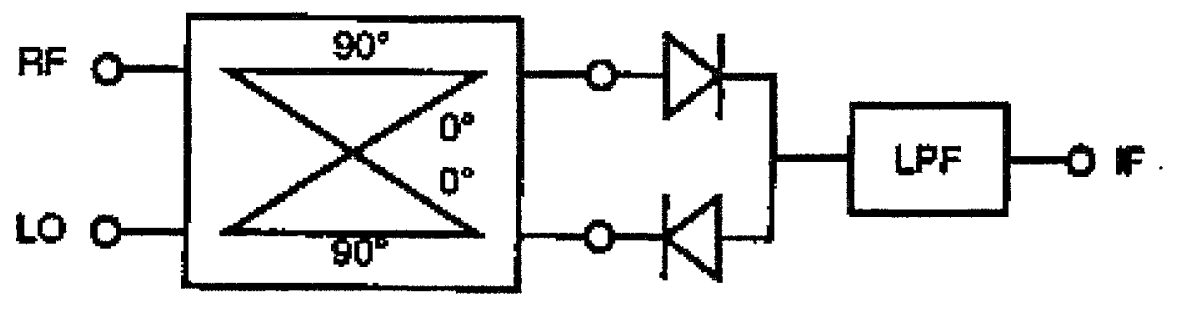

Figure 4-3: Branchline Mixer [11, p.260].

In [19] there are three different branchline coupler mixer topologies. In the "general type" mixer the LO-RF isolation is $8.2 \mathrm{~dB}$. The isolation between the RF and LO comes only from the branchline coupler, whereas the LO/RF-IF isolation is due only to the output filter. The LO-IF isolation is $11 \mathrm{~dB}$, and the RF-IF isolation is $13 \mathrm{~dB}$.

Mixers that are designed based on the topology discussed here using a quadrature coupler have the property of being unilateral, which means that a down-converter cannot be used as an up-converter and vice-versa; an advantage in some applications [11, p. 269]. The disadvantages, however, include narrow bandwidth due to the dependence of the dimensions of the branchline coupler on wavelength, like the ring-hybrid. Size is also an issue, as at lower frequencies the dimensions of the branchline coupler become impractical. The port-to-port isolation in a quadrature coupler is equal to the input return loss seen looking into the two diodes, which is very limiting; leading to poor RF-to-LO isolation [11, p. 268]. Finally, poor return loss at either the RF or LO port unbalances the 
mixer, causing an increase in conversion loss [11 p. 268]. Mixers using quadrature couplers are typically unpopular because of these limitations.

\subsubsection{Marchand Balun and Parallel-Line Baluns:}

The Parallel-Line balun and Marchand balun are in the same family of baluns; the Marchand balun is simply a parallel-line balun that has a stub in series with the load that gives some good properties to the balun. The typical mixer topology involving Marchand or parallel-line baluns can be extracted from [22].

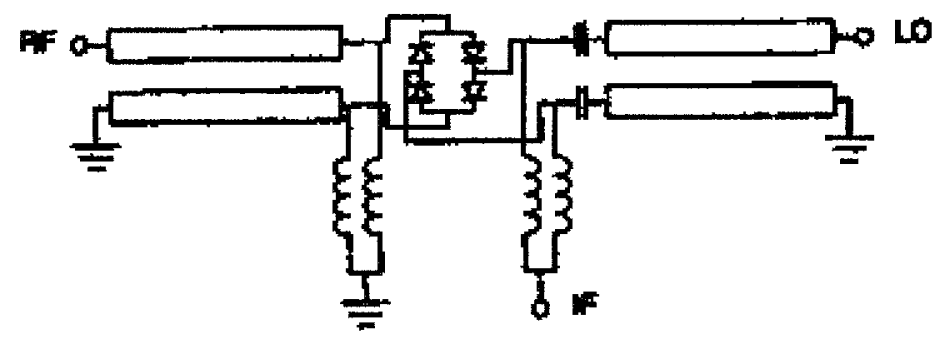

Figure 4-4: Mixer topology using parallel-line baluns [22].

In Figure 4-4, which was extracted from [22], the mixer is double-balanced, so there are actually 2 baluns and 4 diodes. The baluns in Figure 4-4 are parallel line, however the paper describes how the same topology can be implemented using Marchand baluns. A mixer implemented using the above topology with Marchand baluns achieved RF-LO isolation of greater than $30 \mathrm{~dB}$ and an RF-IF isolation of greater than $20 \mathrm{~dB}$ over $12 \mathrm{GHz}$. Conversion losses of around $6 \mathrm{~dB}$ over $12 \mathrm{GHz}$ indicate this is a very efficient planar mixer with a wide bandwidth, due mostly to the properties of the 2 Marchand baluns. Parallel-line baluns are often narrowband due to low even-mode impedance $\left(Z_{o e}\right)[11$, p.258] and because they have only one resonance point. The series stub in the Marchand 
improves bandwidth by adding a second resonance point, as well as increasing the evenmode impedance, $Z_{o e}$, because the load sees two high-impedance $0.25 \lambda$ stubs in series when excited by even-mode currents $[11$, p. 259].

\subsubsection{Mixer topology and layout using new coupler}

The mixer topology used to incorporate the new coupler is illustrated in Figure 4-5 below, along with the final layout in Figure 4-6.

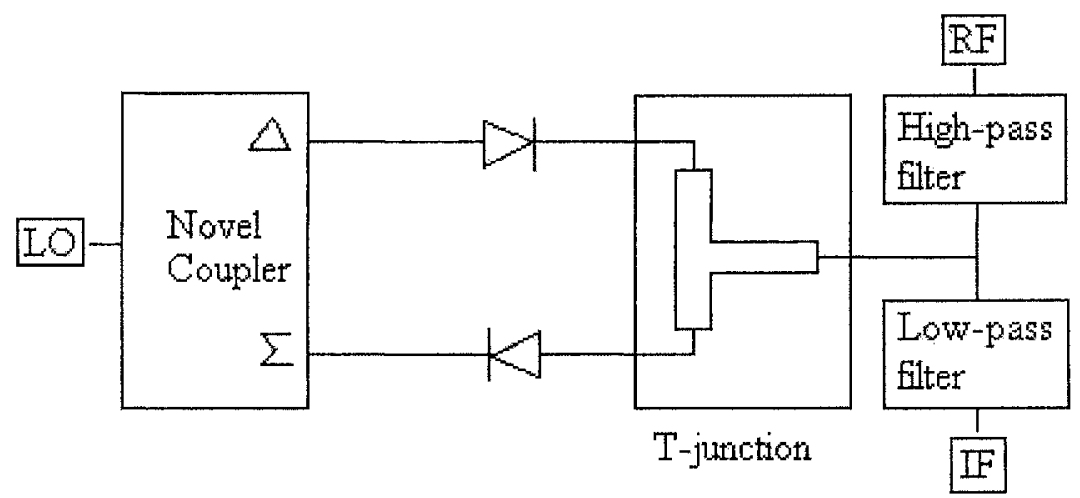

Figure 4-5: Mixer topology including new coupler. 


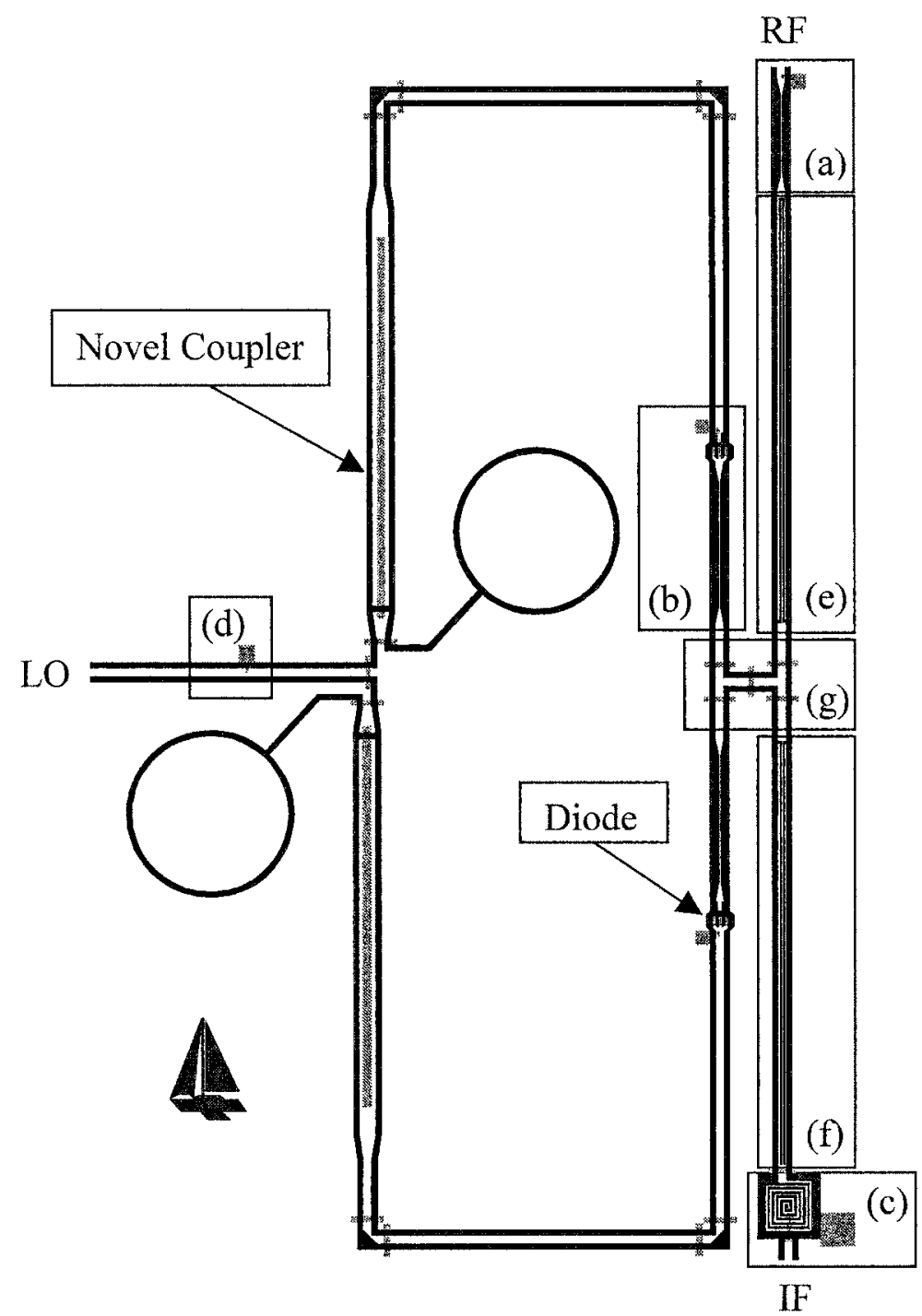

Figure 4-6: Fnal layout using new coupler. 
From Figure 4-5, we note the essential components of the topology are:

- The new coupler, which splits the LO power into output signals separated by $180^{\circ}$ of phase shift.

- The diodes, which are placed in the ring created by the new coupler and a Tjunction.

- The T-junction, which supplies the RF signal, as well as extracts the IF signal.

- A high-pass filter blocking IF signal from interfering with the RF port.

- A low-pass filter blocking the RF signal from interfering with the IF port.

- Matching circuitry (not shown in topology).

In Figure 4-6 the layout has been segmented into the major components. These are:
(a) RF matching structures
(b) Diode matching structures
(c) IF matching structures
(d) LO matching capacitor
(e) High-pass filter
(f) Low-pass filter
(g) Double T-junction

The advantages that the new coupler lends in the above topology are the fact that the coupler is simple to design and the outputs of the coupler are essentially independent so long as the electrical length between the output ports are such that the $180^{\circ}$ phase shift is maintained. The physical characteristics of the coupler, with its independent output tracks and straightforward manipulation of the outputs ports, make it an efficient user of space, while other couplers, like the ring-hybrid and branchline coupler, can leave a large footprint of unusable circuit space in the center. Finally, the inherent phase balance of the coupler is helpful in ensuring efficient mixing in the two diodes. In fact, the characteristic of inherent phase balance of the novel coupler makes it possible to design a mixer with a large frequency spread - i.e. $5 \mathrm{GHz}$ - between the $\mathrm{LO}$ and RF frequencies. 
A mixer with a frequency spread of $5 \mathrm{GHz}$ could be used to down-convert a $28 \mathrm{GHz}$ LMDS channel directly to a $5 \mathrm{GHz}$ unlicensed band used by 802.11 a equipment, creating a combined wireless backhaul network (LMDS) and wireless subscriber local area network (802.11a). It is difficult if not impossible to design efficient mixers with large frequency spreads using ring hybrid couplers, branchline hybrid couplers, coupled line baluns, or Marchand baluns. For each of these other couplers, the phase balance is linked to the electrical dimensions of the structure, which are dependant on frequency. Therefore if these other couplers are designed to have an excellent phase balance at the LO frequency (for example), the phase balance at the RF frequency is doomed to be poor. This severely limits the amount of frequency-spread possible.

Simulation results can be found at the end of this chapter, however it is useful to note that the topology yields simulated LO-RF isolation of $33.4 \mathrm{~dB}$ (provided by the coupler and the high-pass filter at the RF port), RF-LO isolation of $28 \mathrm{~dB}$ (provided by the coupler only). One major advantage of the new coupler as used in the mixer topology is the fact that the simulated LO to IF isolation is $73.0 \mathrm{~dB}$ (due to the coupler and the low-pass filter), a rare quality for a mixer to have. Typically there is only a filter isolating the IF port from the powerful LO signals, however because of the new coupler the signals arriving at the $\mathrm{T}$-junction are $180^{\circ}$ apart, attenuating the LO signal before the signal arrives at the low-pass filter, which further reduces the LO signal. Finally the simulated RF to IF isolation is $55.1 \mathrm{~dB}$. 
The disadvantages of applying the new coupler in the above mixer topology are the fact that it is impossible to perfectly match the three ports of a loss-less, reciprocal 3-port network [16], and the fact that while the bandwidth of the coupler may be wide, the bandwidth of the mixer is limited by the filters and matching networks.

\subsection{Details of Mixer Design Using the New Coupler}

\subsubsection{Diode Selection and Sizing:}

The single balanced mixer design began with a test circuit incorporating the new coupler that was designed for this thesis along with two diodes from the OMMIC ${ }^{\mathrm{TM}} \mathrm{ED} 02 \mathrm{AH}$ library. Figure 4-6 of this test circuit is shown below.

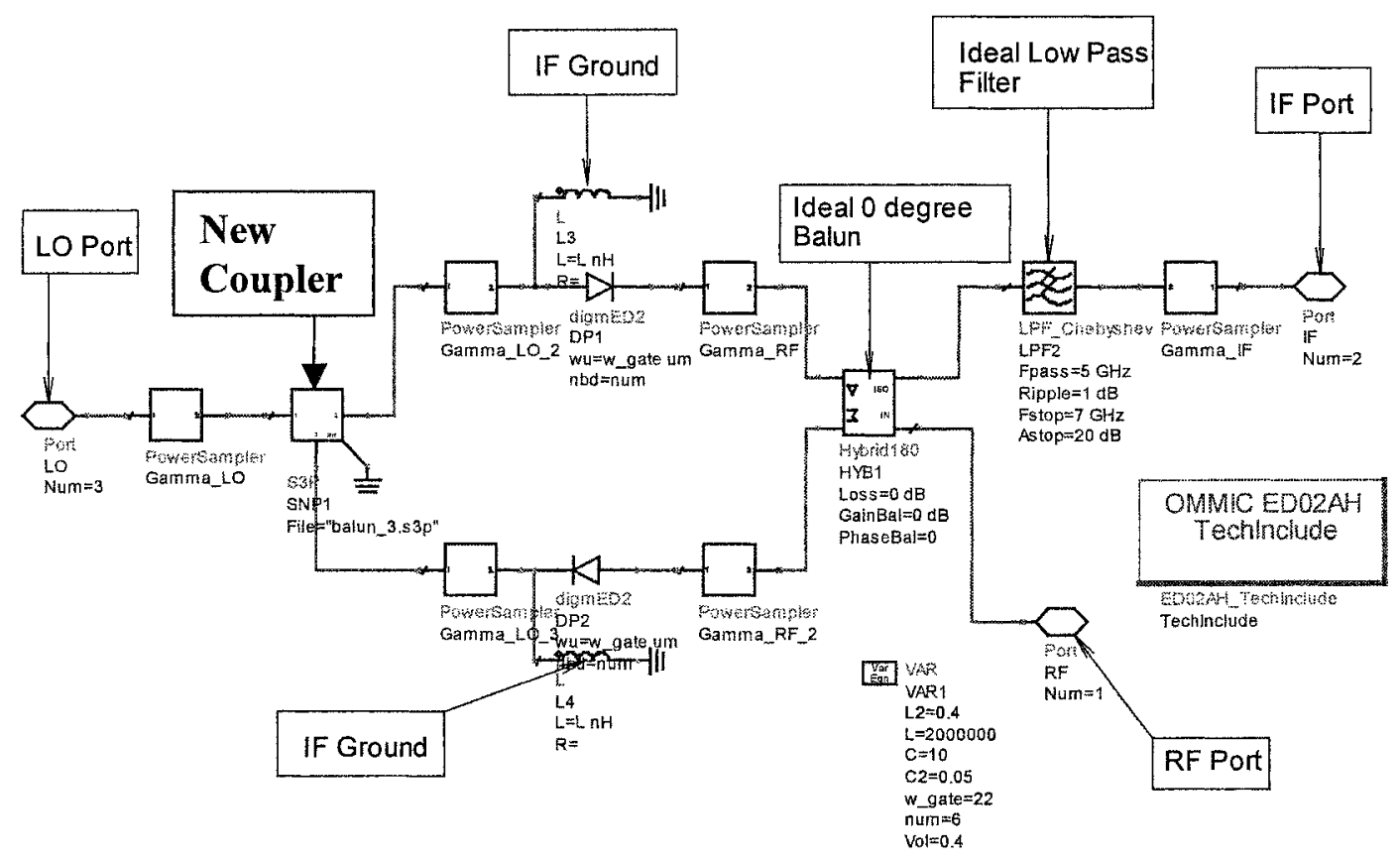

Figure 4-7: Test circuit with new coupler and diodes from the OMMIC $^{\mathrm{TM}}$ EDO2AH library. 
The test circuit in Figure 4-6 has an ideal power splitter, which is simply a 0 degree phase shift coupler for delivering the RF signal to the diodes and extracting the IF signal. There is also an ideal low-pass filter going to the IF port. Finally, a pair of inductors with very large inductances are used as ideal IF grounds. The circuit in Figure 4-6 above was put together simply to prove that the configuration using the new coupler could deliver the LO and RF power to the diodes for mixing, as well as be able to extract the IF. This circuit was also used to sweep the values of LO power and the diode characteristics to produce the most efficient mixing.

The diodes selected from the OMMIC ${ }^{\mathrm{TM}} \mathrm{ED} 02 \mathrm{AH}$ library were the diodes called ' $\mathrm{GM}$ ' diodes in the OMMIC ${ }^{\mathrm{TM}}$ documentation [23]. The 'GM' diodes are P-HEMT diodes, where HEMT stands for High Electron Mobility Transistors. In OMMIC ${ }^{\mathrm{TM}}$ 's proprietary fabrication process, a GaAs HEMT is fabricated with a thin layer of undoped InGaAs, which is a material with very high electron mobility and saturation velocity. This property gives HEMTs better gain (in transistors) and noise characteristics, as well as a faster switching response. Also the transconductance of a HEMT diode rises more sharply with increasing gate voltage than a typical diode without the InGaAs layer, and has a distinct peak [11, p.71]. This suggests that HEMTs are more strongly nonlinear, which is ideal for mixers. More nonlinearity means less LO power is needed to achieve efficient mixing, which translates to lower conversion loss.

The 'GM' diodes are also characterized as Schottky-barrier devices. Schottky diodes are fabricated by depositing metal directly on a semiconductor, rather than using two doped 
semiconductor materials to produce a PN junction. The Schottky diode is a majority carrier device, unlike a PN junction, which is a minority carrier device. Therefore the Schottky diode has faster switching speed than a PN junction diode, since minority carrier devices suffer from some effects that slow switching [11, p.11]. Finally, Schottky diodes are easier to manufacture with a high degree of consistency [12, p.41]. Due to these advantages, Schottky diodes are universal in high frequency circuits.

The mixer test circuit in Figure 4-7 above was put into a harmonic balance test-bench, which is shown in the Figure 4-8 below:

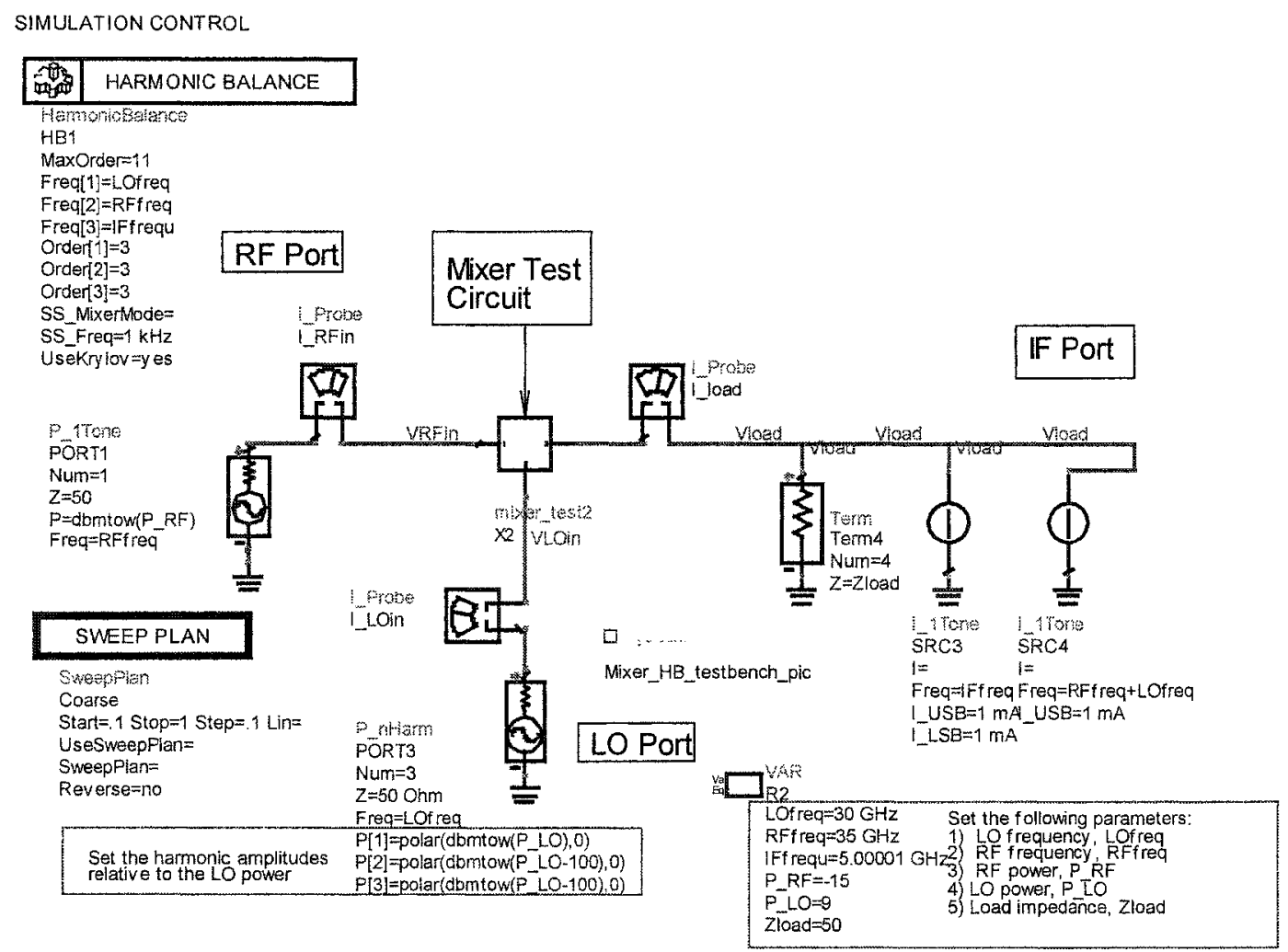

Figure 4-8: Harmonic Balance Test-Bench.

Harmonic balance simulations are simulations where the effects of multiple frequency sources on a circuit are modeled, whether these frequency sources come from direct input 
or through the creation of harmonics from nonlinear elements in the circuit. In Figure 4-8 there are two direct input frequency sources - one at the RF port, and one at the LO port. The two current sources at the IF port exist purely for injecting power for measuring return loss and isolation at that port, and are not directly involved in the mixing process.

For the harmonic balance simulation, the RF source was given an order of 3 , while the LO source was given an order of 11. The order represents the depth of harmonics, or spectral resolution, used in the simulation. The higher the order set, the more accurate the simulations will be, but the time it takes for the simulations to converge will increase as a consequence. The order of the RF and LO sources were default settings for the example mixer test-bench supplied by the $A D S^{T M}$ software, but this is also intuitive in the sense that because the LO power is very high, its harmonics will have the most effect on the diodes and, as a consequence, on the frequency response of the mixer. So it makes sense that one would want a higher resolution in modeling the LO harmonics than the RF harmonics. The first simulation was to sweep the LO power to see which input power provided the highest conversion gain, where conversion gain is defined as: $G_{c}=\frac{P_{I F}}{P_{R F}}$ Where $\mathrm{P}_{\mathrm{RF}}$ is the RF input power, and $\mathrm{P}_{\mathrm{IF}}$ is the IF output power.

It is clear that in the above definition the LO input power does not play a direct role in the calculation of conversion gain, however the level of LO input power does impact the conversion gain directly, as shown in the graph of conversion gain versus input LO power given in Figure 4-9 below. 


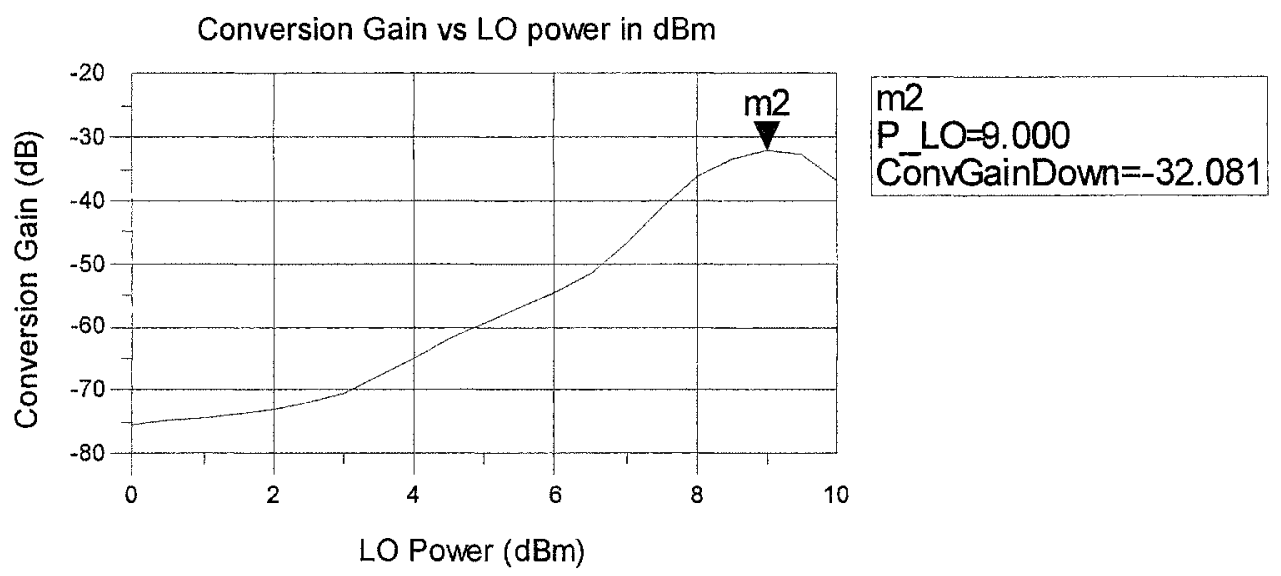

Figure 4-9: Conversion Gain versus Input LO Power.

Since this is a diode mixer, a conversion loss is measured as opposed to a conversion gain, since diodes do not provide current or voltage gain like transistors do. From Figure 4-9 we see that the minimum conversion loss of $32 \mathrm{~dB}$ occurs for an input LO power of 9 $\mathrm{dBm}$. It is important to note that the very poor value of $32 \mathrm{~dB}$ of conversion loss is due to the fact that no work had been done on matching at this point.

The reason there exists an optimum operating point for the input LO power is because there is a relationship of maximum non-linearity to LO power in the I-V curve of the diode. Too little LO power, and the peak voltage swing is not yet saturating the diode enough, so there isn't enough power transferred to the harmonics for mixing. Too much LO power and the signal is oscillating mostly in the linear region of the diode, meaning that little distortion to the envelope of the LO signal exists. Between these two limits, an 
ideal point exists for maximum conversion efficiency. The I-V curve for the 'GM' diodes used in the simulation is given in Figure 4-10 below.

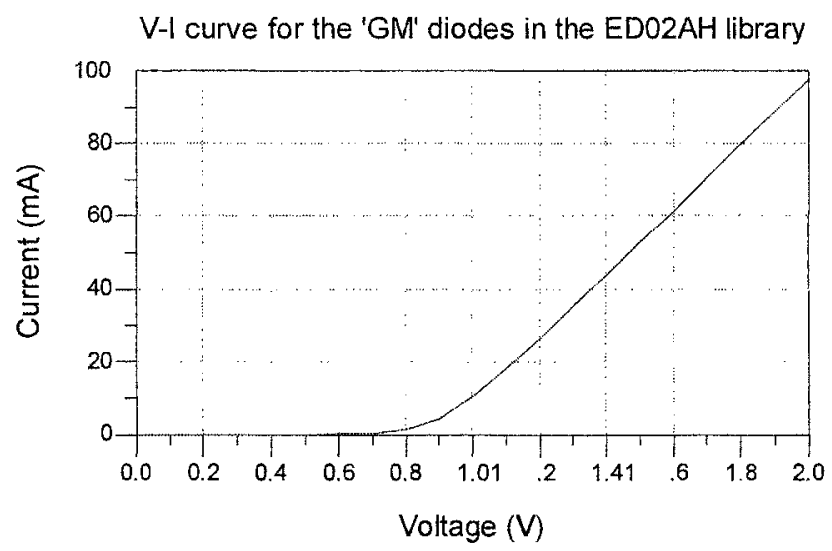

Figure 4-10: V-I curve for the 'GM' diodes used in the mixer test circuit.

From Figure 4-10, observe that the corner voltage for this diode is $0.9 \mathrm{~V}$. The corner voltage is the center of the most nonlinear part of the I-V curve. Therefore the optimum input LO power will be such that the LO waveform will oscillate about the $0.9 \mathrm{~V}$ corner voltage, which from Figure 4-9 above occurs when the LO power is $9 \mathrm{dBm}$.

The RF input power was also swept in order to make sure that an appropriate input power was selected such as not to be in the saturation region. The saturation region occurs for the RF input power because initially most of the power is delivered to the fundamental frequency for mixing, but as power increases, the signal is compressed by nonlinearity in the diodes causing an increase in the power delivered to the third harmonic [24]. Eventually, at some RF input power the power in the third harmonic, because it increases by the square, will overtake the power in the fundamental frequency. The point where 
the power in the third harmonic overtakes the power in the fundamental frequency is named the third order intercept point [24], and the consequence of this increase in power to the third harmonic means less and less power is left in the fundamental tone for mixing. This leads to an eventual drop in the conversion gain.

Figure 4-11 shows the result of sweeping RF input power.

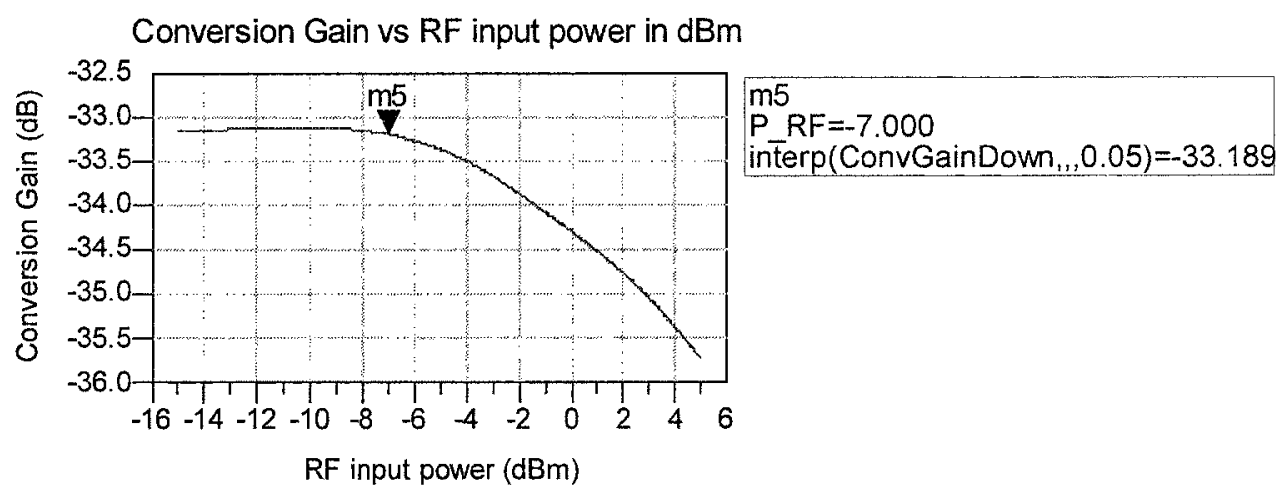

Figure 4-11: Conversion Gain versus RF input power.

As Figure 4-11 shows, an RF power of up to $-7 \mathrm{dBm}$ can be selected without suffering increased conversion loss of more than $1 \mathrm{~dB}$. For the remainder of simulations, an RF input power of $-15 \mathrm{dBm}$ was used.

Next, the number and length of the gate fingers in the diode were swept to find the optimal diode physical characteristics for mixing. The Figure 4-12 below shows the effect on conversion gain of sweeping the gate finger length from 17 to $28 \mu \mathrm{m}$. 


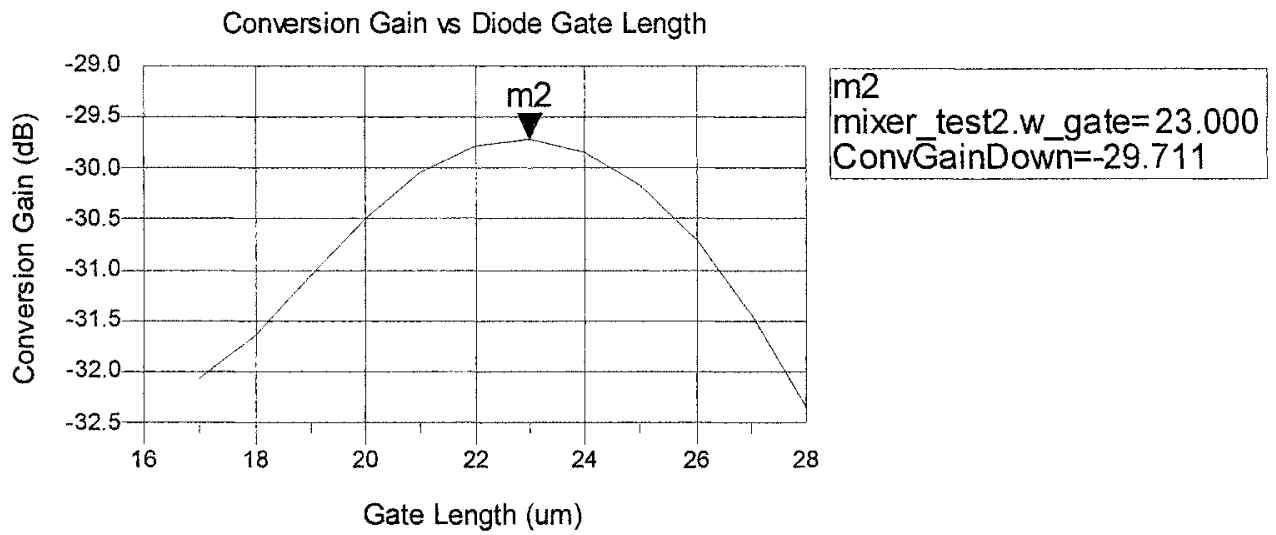

Figure 4-12: Conversion gain versus finger length.

Figure 4-12 clearly shows that a minimum conversion loss of $-29.71 \mathrm{~dB}$ exists for a gate finger length of $23 \mu \mathrm{m}$. Setting the gate finger length to $23 \mu \mathrm{m}$ and now sweeping the number of fingers in the diode gate, the following figure is produced.

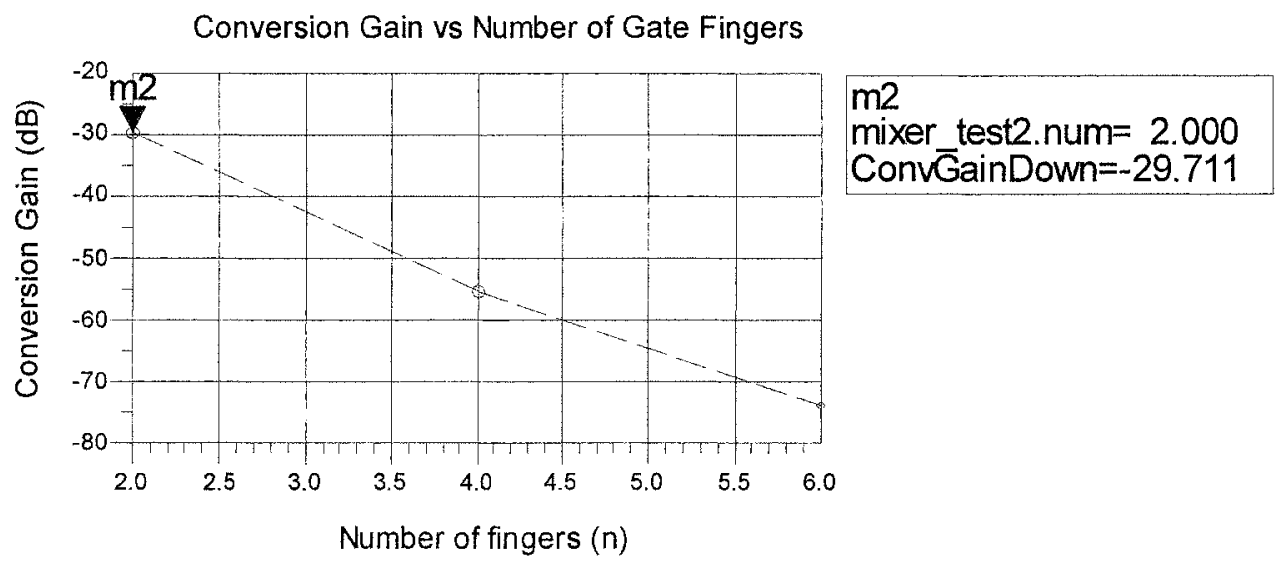

Figure 4-13: Conversion gain versus number of fingers. 
Note that the number of diode fingers must be a multiple of 2 and can only go from 2 to 6. Therefore there are only three data points in the above graph, along with a trend line. It is clear from Figure 4-13 that the minimum conversion loss occurs for the lowest number of fingers - which is 2 .

The reasons for the behavior of the mixer with varying diode characteristics can be explained by observing the equivalent model of the OMMIC ${ }^{\mathrm{TM}} \mathrm{ED} 02 \mathrm{AH}$ 'GM' diode, which is shown in Figure 4-14 below.

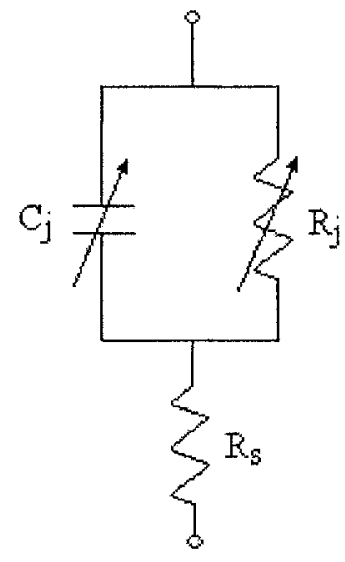

Figure 4-14: Equivalent circuit of 'GM' diode [25, p.318].

A discussion on conversion gain, as it relates to the elements of the equivalent circuit in Figure 4-14, lends insight into the observed simulation behavior. The ideal conversion loss is $3 \mathrm{~dB}$ due to the fact that only one of the sidebands is extracted in a single-sideband mixer, so half the power is lost in the image sideband that is discarded. One expression for the degradation in conversion-loss from the ideal is as follows [25, p.319]. 
Degradation at the RF frequency:

$\delta_{1}=1+R_{S} / r_{d r}+R_{S} r_{d r}\left(w C_{j 0}\right)^{2}$

Where:- $R_{S}$ is the diode series resistance

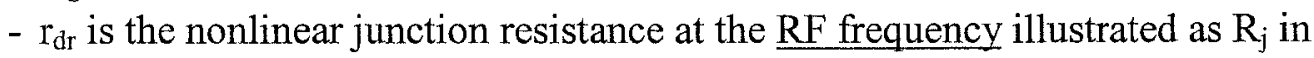
Figure 4-14 above.

$-w$ is the angular RF frequency.

$-\mathrm{C}_{\mathrm{j} 0}$ is the variable capacitor in Figure 4-14 above.

It can be extracted from equation 4-1 that there is an optimum size for the diode for a given frequency. This is because "the relationship between $R_{S}$ and $C_{j 0}$ for any diode geometry is inversely related; that is, as the junction area is reduced so that $\mathrm{C}_{\mathrm{j} 0}$ can be minimized, $\mathrm{R}_{\mathrm{S}}$ increases." $[26, \mathrm{p} .531]$ This correlates with the observed maxima seen in Figure 4-12 above when sweeping diode gate dimensions.

\subsubsection{Matching:}

Having found the initial dimensions of the diodes and LO input power, explorations into how to match the junctions in the mixer circuit can begin. The important junctions of the mixer circuit are:

- Input from LO port into the coupler. (refer to Figure 4-6(d))

- Input from the RF port into the T-Junction. (refer to Figure 4-6(a))

- Output to IF port from T-junction (refer to Figure 4-6(c))

- Junction between both coupler outputs and the two diodes. (refer to Figure 4-6(b))

- Junction between outputs of T-junction and the two diodes. (refer to Figure 4-6(b)) 
A number of tools and strategies were used in matching, which include:

i. Using non-invasive dual power samplers to give reflection and impedance at all junctions in the layout.

Dual power samplers are the boxes labeled PowerSampler in Figure 4-6.

They are directional measuring elements that measure the power that enters the box, the power that is reflected, and the power that is transmitted, all at a specific frequency. They are used for matching internal circuit structures by yielding reflection and transmission loss that otherwise could not be obtained using a port, since port terminations cannot be used internally in a circuit simulation. They are 'invisible' to the circuit - i.e. they do not impact how the circuit functions in simulation.

ii. Using test circuits to create a perfect match for the conjugate of the measured input impedance $\rightarrow$ typically with ideal lumped elements that are later exchanged for real circuit elements in the final layout. This matching circuit was then re-introduced to the mixer circuit directly. (Applied to ports: LO, $R F$, and $I F)$

Refer to [10] for information on extracting the measured input impedance from a Smith Chart, and designing conjugate matching circuits using ideal lumped elements. Refer to Figure 4-6(a), (b), (c), and (d) for the sections highlighting the fabricated lumped capacitors and inductors that were translated from ideal elements. 
iii. Using lengths of transmission line that have different characteristic impedances in order to transform the impedance (inner ring of mixer).

Reference $[10, p .83]$ explains the concept of the quarter wavelength transformer. Essentially, if one wishes to match a section of transmission line at a lower impedance (say $50 \Omega$ ) to a section of transmission line with a higher impedance (say $100 \Omega$ ), one needs a quarter-wavelength long line that has an intermediate impedance defined as

$Z_{t x}=\sqrt{Z_{1} Z_{2}}=\sqrt{50 * 100}=70.71 \Omega$

As shown, a transformer line impedance of $70.71 \Omega$ is required to match a 50 $\Omega$ line to a $100 \Omega$ line. This matching concept is visible in Figure 4-6(a) and (b), where a thin line with a high impedance was used to match the RF port and to match the diodes to the T-junction.

iv. Parameter tweaking: by using ideal matching elements that are thought to aid matching, parameters are adjusted and the results are observed on a Smith Chart. Establishing trends, parameters are changed to get the best possible reflection coefficients.

This trial and error method was necessary for optimization for part of the design because of the fact that one is trying to match a linear, loss-less, reciprocal, 3-port network with no isolation between output ports, where a perfect match is impossible [16]. This makes the matching process for the inner ring of the mixer, the transmission line loop containing the diodes, 
unpredictable, as there was not a closed-form solution for generating the matching networks for the mixer. The lumped capacitor and high impedance line in Figure 4-6(b) constitute the matching structures partially derived from this technique.

v. Use of shunt short and open circuit stubs to create resonant matching structures.

These were initially used to match the internal junctions between the coupler and the diodes of the mixer, however this was problematic because of the difficulty in matching a 3-port network as discussed previously; designing and simulating the structures with stubs takes a long time, which was compounded by the fact that the matching process required many iterations. In the end more energy was delivered to the diodes by laying out transmission lines of varying impedance directly to the diodes.

Figure 4-15 illustrates the final matching structures used in the mixer, and are matched to the segments in Figure 4-6 seen previously. 


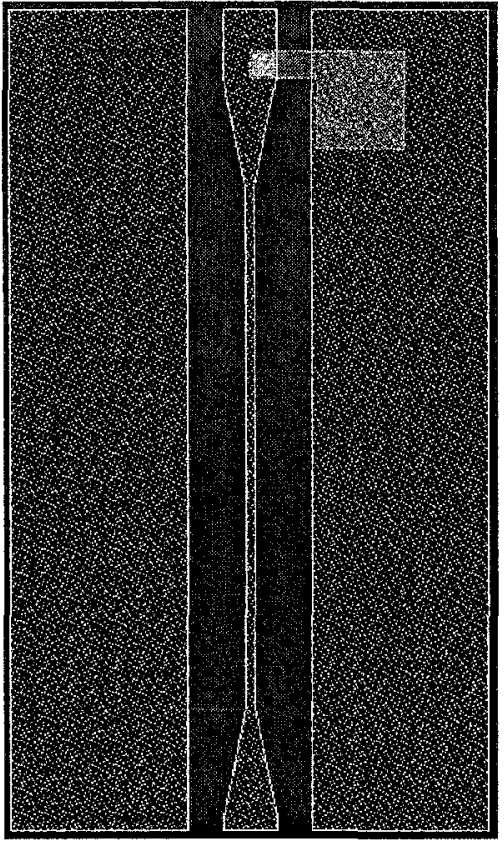

(a)

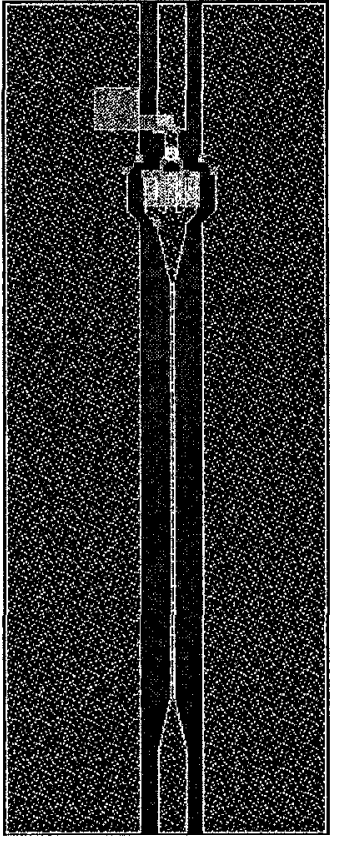

(b)

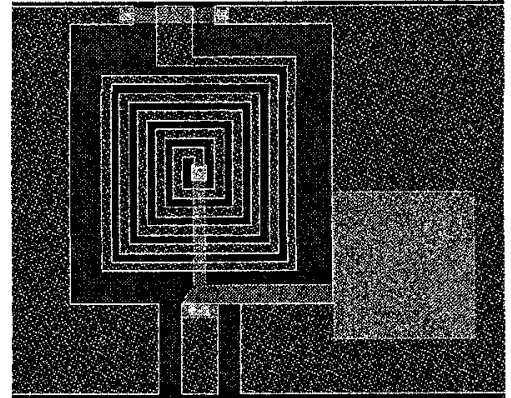

(c)

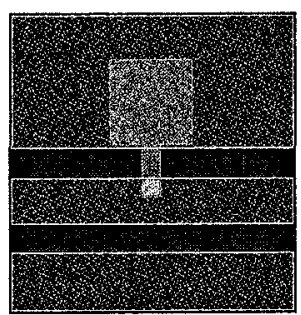

(d)

Figure 4-15: a) matching circuit at RF port, b) matching circuits at the diodes, c) matching circuit at the IF port, and d) matching shunt capacitor at LO port.

Figure 4-15(a) above shows the matching circuit at the RF input port of the mixer. It consists of a shunt capacitor and a narrow, impedance-transforming piece of transmission line. The high-impedance transmission line is not quite a quarter-wavelength long, however it serves the purpose of shifting the impedance clockwise on the Smith Chart to a position where a shunt capacitor of reasonable size brings the impedance very close to $50 \Omega$. If one refers to Figure 4-7, one will find a number of boxes in the schematic dubbed "PowerSampler". Each of these boxes is named, Gamma_LO at the LO port for example, and represents a point in the mixer where reflection coefficients were measured during the matching process. Figure 4-16 below shows a Smith Chart plotting all the matching points of interest in the mixer, with Gamma_RF1 representing the point that 
shows the effectiveness of the RF input matching circuit with a simulated return loss of $-27.25 \mathrm{~dB}$, recorded in Table 4-1 below.

Figure 4-15(b) above shows the matching structures used inside the diode ring to transfer maximum power to the diodes. The structures consist of a shunt capacitor and a length of high-impedance transmission line. The difficulty for the matching inside the diode loop is that the matching must work in one direction at the LO frequency, while also being a good match for the RF frequency in the other direction. It is often the case that the matching at the RF frequency is sacrificed somewhat for a better match at the LO frequency. This is logical since it is the high-powered LO signal that essentially drives the diodes and contributes most to mixer conversion gain. In the Smith Chart of Figure 4-16 below, the compromise between LO and RF frequency matching in the diode ring is illustrated. The points Gamma_LO_2 and Gamma_LO_3 just up and to the left of the center of the Smith Chart show the LO match, at better than $-12 \mathrm{~dB}$ of return loss in simulation, while the RF match, the points Gamma_RF and Gamma_RF2, is far down and to the right on the Smith Chart with a terrible return loss of $-3 \mathrm{~dB}$. It is this compromise on matching that yielded the best conversion gain in the mixer. It is important to separate the LO and RF matching done within the diode ring, and the LO and RF matching done at the LO and RF ports. The LO and RF ports are buffered from the impedances in the diode ring by the coupler and the T-junction, i.e. for the LO signal the circuit beyond the coupler is seen as a virtual ground. Likewise for the RF port, the circuit beyond the T-junction is seen as a virtual ground. For this reason it is possible for the match seen at the actual ports to be much better than what is seen within the diode 
ring itself, the RF match being a good example with a match of $-27.25 \mathrm{~dB}$ seen at the RF port, however the RF match within the diode ring is $-3 \mathrm{~dB}$.

Figure 4-15(c) above shows the matching circuit at the output IF port. It consists of lumped components, since distributed components at the IF frequency of $5 \mathrm{GHz}$ would be unrealistically large. The IF matching circuit has a series inductor and a shunt capacitor that yield a very good simulated match of $-30.65 \mathrm{~dB}$. The IF matching point on the Smith Chart in the Figure 4-16 below is named Gamma_IF and lands directly on the center of the Smith Chart.

Figure 4-15(d) above shows the final matching circuit found at the input LO port. It is simply a small shunt capacitor, since in the end this was all that was needed to move the LO match very close to the center of the Smith Chart and yield a simulated return loss of $-21.54 \mathrm{~dB}$. The point Gamma_LO in the Smith Chart of Figure 4-16 below shows the input LO match just above the center of the chart. 


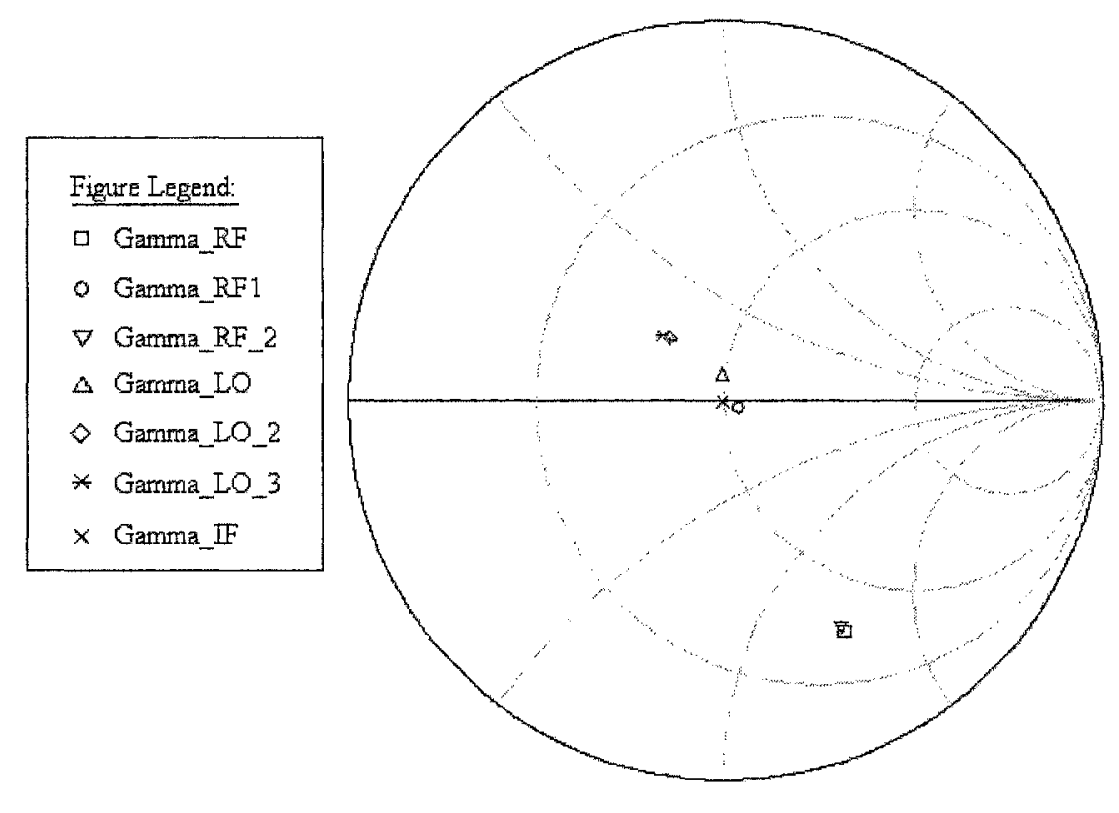

Figure 4-16: Smith Chart of matching in mixer

Finally, table 4-1 summarizes the final simulated reflection coefficients at the three ports of the mixer.

Table 4-1: Reflection coefficients at the LO, RF, and IF ports.

\begin{tabular}{|lc|}
\hline \multicolumn{1}{|c|}{ Port } & $\begin{array}{c}\text { Reflection } \\
\text { Coefficient }(\mathbf{d B})\end{array}$ \\
\hline RF port (frequency $=35 \mathrm{GHz})$ & -27.25 \\
LO port $($ frequency $=30 \mathrm{GHz})$ & -21.54 \\
IF port $($ frequency $=5 \mathrm{GHz})$ & -30.65 \\
\hline
\end{tabular}

\subsubsection{Filters:}

While isolation is implicit in between the LO and RF ports, or the LO and IF ports, due to the coupler, the same is not true for the isolation between the RF and IF ports. Thus some form of filtering was necessary at both ports. A high-pass filter was designed for 
the RF port, and a low-pass filter was designed for the IF port. The Figure 4-17 below shows the high-pass filter used at the RF port.

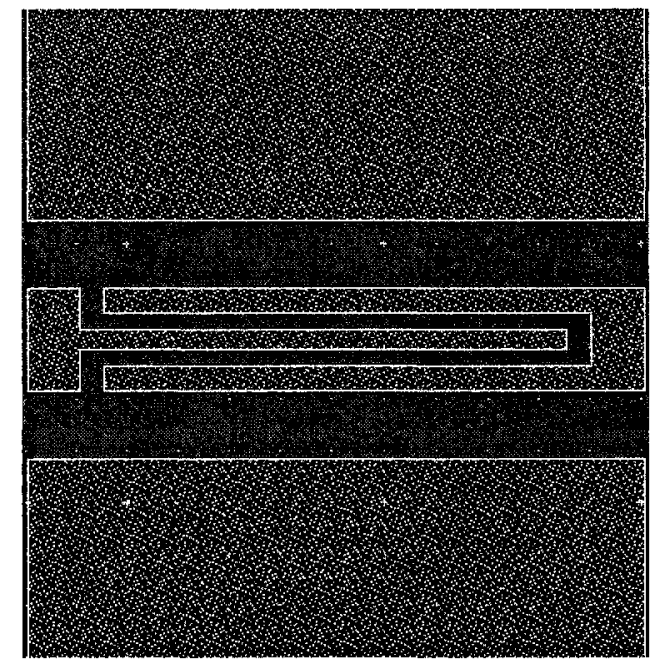

Figure 4-17: High-pass filter.

Refer to Figure 4- $\dot{6}(\mathrm{e})$ to show where the high-pass filter is situated in the circuit. Note that the real stub is $995 \mu \mathrm{m}$ long, which is too long to see the profile of the series stub, therefore it has been shrunk for Figure 4-17. From the above Figure we see clearly that the filter is simply a series open stub that is a quarter-wavelength tuned to allow $35 \mathrm{GHz}$ to pass. The simulated S-parameters for the above HPF are given below. 


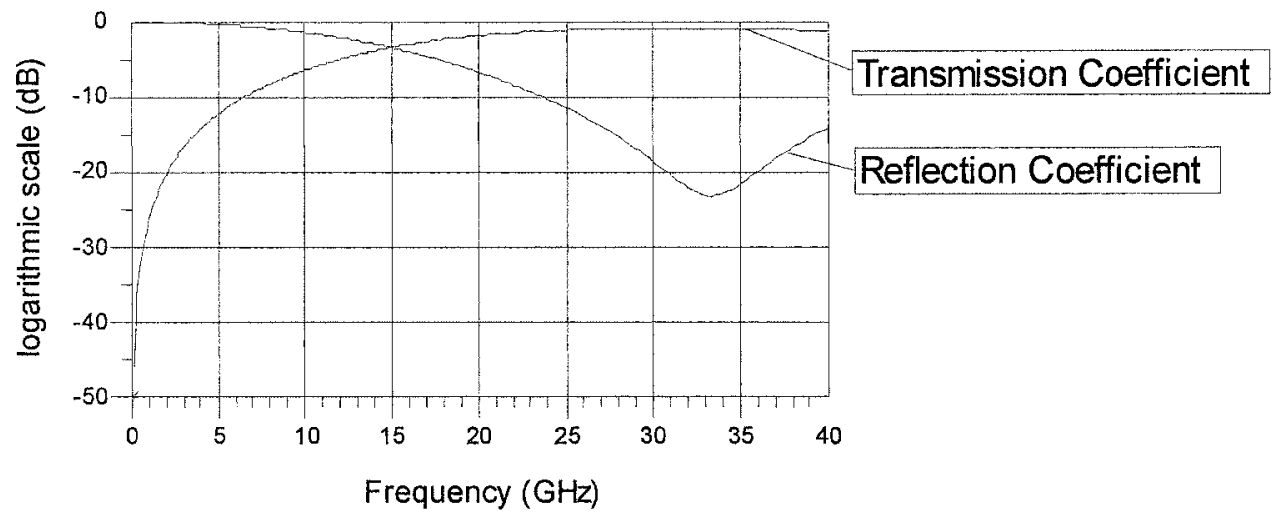

Figure 4-18: Simulated s-parameter results for high-pass filter.

In Figure 4-18, the transmission coefficient at $35 \mathrm{GHz}$ (the RF channel) is $-0.95 \mathrm{~dB}$, meaning that the filter allows the RF channel to pass with less than a $1 \mathrm{~dB}$ of transmission loss. At the IF frequency of $5 \mathrm{GHz}$, the transmission coefficient is -12.23 $\mathrm{dB}$, which is the level of isolation from the IF frequency offered by the filter at the RF port.

Similarly, a low-pass filter was created using a series shunt stub that is a quarter wavelength again tuned to $35 \mathrm{GHz}$, this time to attenuate it. Figure 4-19 of the low-pass filter is given below. 


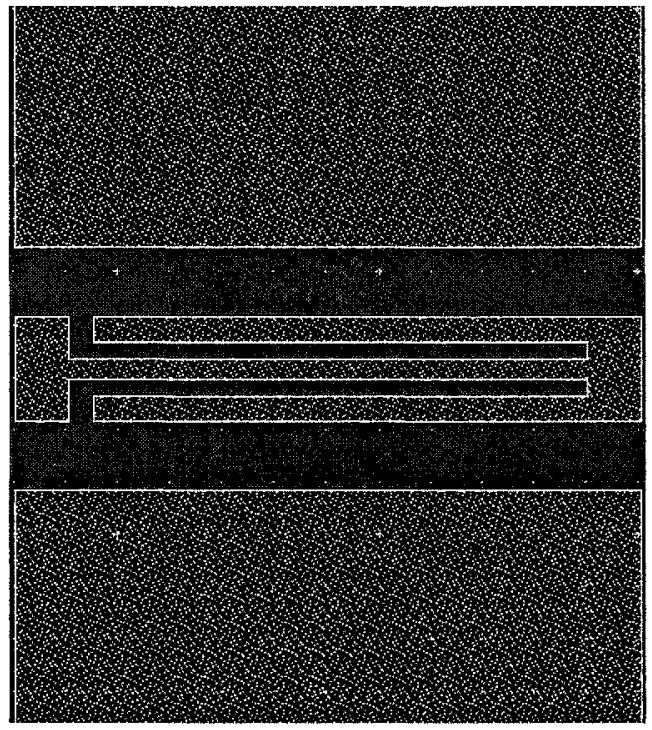

Figure 4-19: Low-pass filter.

Refer to Figure 4-6(f) to show where the low-pass filter is situated in the circuit. The simulated S-parameters for the low-pass filter are given below:

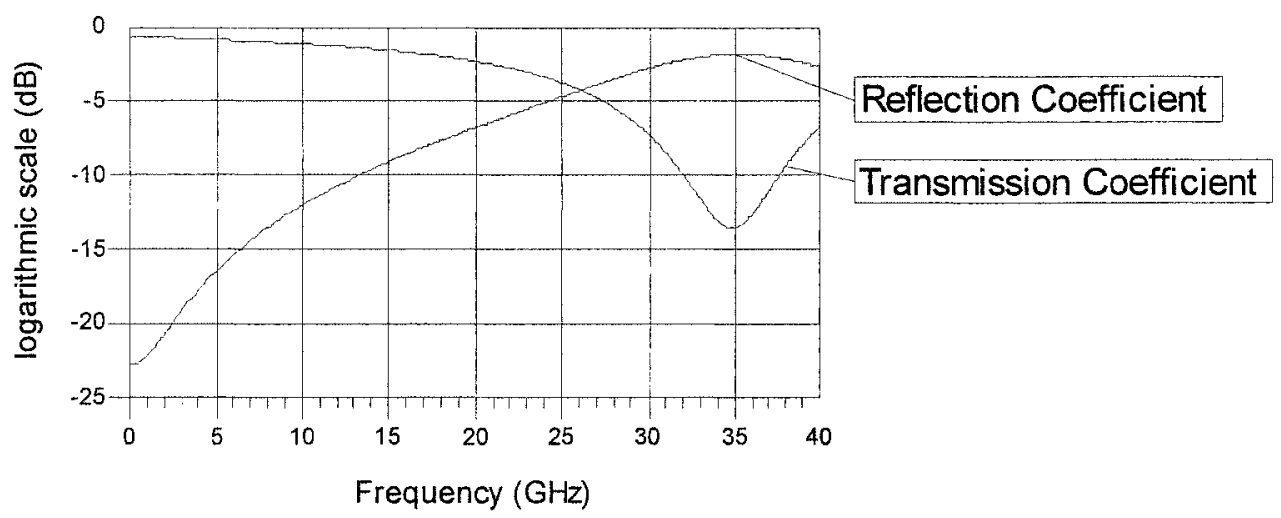

Figure 4-20: Simulated s-parameter results for low-pass filter.

In Figure 4-20, the transmission coefficient at $5 \mathrm{GHz}$ (the IF channel) is $-0.83 \mathrm{~dB}$, meaning that the filter allows the IF channel to pass with less than a $1 \mathrm{~dB}$ of transmission 
loss. At the RF frequency of $35 \mathrm{GHz}$, the transmission coefficient is $-13.57 \mathrm{~dB}$, which is the level of isolation from the RF frequency offered by the filter at the IF port.

\subsection{Simulation Results}

Sections 4.1 and 4.2 of this chapter described the various elements of the single-balanced mixer design. The following figures and paragraphs describe the simulated performance results of the mixer. Figure 4-21 gives the conversion gain as well as port-to-port isolation for the three ports.

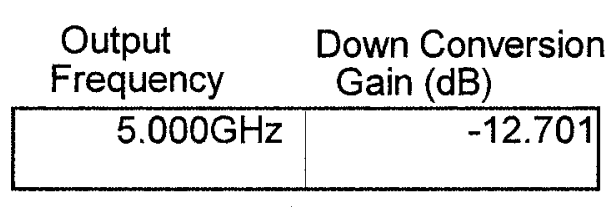

(a)

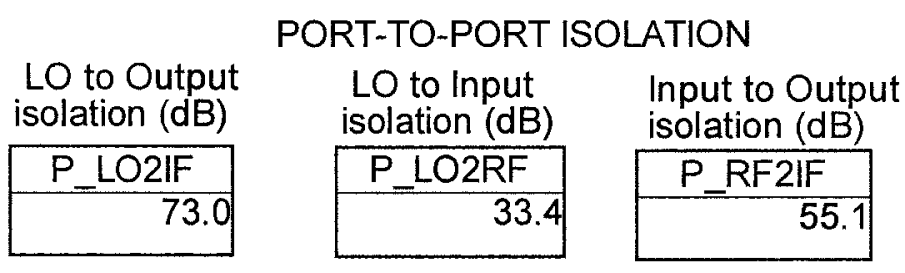

(b)

Figure 4-21: (a) conversion gain and (b) port-to-port isolation.

Figure 4-21(a) shows that the mixer actually has a conversion loss, since this is a diode mixer, of $12.7 \mathrm{~dB}$. This level of conversion loss is high, but it is still reasonable for a planar mixer, which tend to be lossier than other technologies, such as rectangular waveguides. Rectangular waveguide mixers have been developed with conversion losses 
that approach the intrinsic conversion loss limits of the employed Schottky diodes [28]. Intrinsic conversion loss can be between 3 and $4 \mathrm{~dB}$, depending on the device and frequency of operation [29]. To correlate the simulated conversion loss with theoretical estimation, the following calculations were made following the expressions in [27]:

The amount of conversion loss can be summed as follows:

Loss $(\mathrm{dB})=$ Conversion loss + Transformer loss

$$
+ \text { Harmonic losses + Diode losses [27, p. 639] }
$$

Conversion loss is $3 \mathrm{~dB}$, in the ideal case, and represents the power lost in the image frequency during filtering, since only one of the sideband frequencies is extracted in a single-sideband (SSB) mixer. Transformer loss represents the power lost in the coupler of the balanced mixer, and is measured to be $5 \mathrm{~dB}$ from the simulated $S_{21}$ of the final coupler design from Figure 3-33 of chapter 3. Harmonic losses represent the power lost in producing the various n-order harmonics that are not used in the mixing process, and is estimated here by rule of thumb to total $1 \mathrm{~dB}$. Diode losses represent the losses in the series $R_{S}$ resistor of the diode model of Figure 4-14 in section 4.2.1 of this chapter, and can be estimated as follows $[27, \mathrm{p} .640]$ :

Diode_Loss $(d B)=\log _{10}\left(\frac{50+\left(2 \times R_{S}\right)}{50}\right)=\log _{10}\left(\frac{50+(2 \times 17.93)}{50}\right)=2.348$

$\mathrm{R}_{\mathrm{S}}$ was calculated for the above equation to be $17.93 \Omega$ for a diode with a gate finger width of $23 \mu \mathrm{m}$ and the number of fingers equal to 2 . The exact method for calculating 
$\mathrm{R}_{\mathrm{S}}$ is confidential to the process used for design; for more information contact the $\mathrm{OMMIC}^{\mathrm{TM}}$ foundry [23]. The estimate for conversion loss from equation 4-4 is therefore:

$\operatorname{Loss}(\mathrm{dB})=3+5+1+2.348=11.348 \mathrm{~dB}$

This estimation is less than $1 \mathrm{~dB}$ from the actual simulated conversion loss of $12.231 \mathrm{~dB}$, with the difference easily attributable to line losses, which are not factored into the expression.

Figure 4-21(b) shows high levels of isolation, especially between the LO and IF port, which is a property unique to this mixer design and is attributed to the topology using the novel coupler. The next figure shows the spectrum seen at the IF port from the harmonic balance simulation.

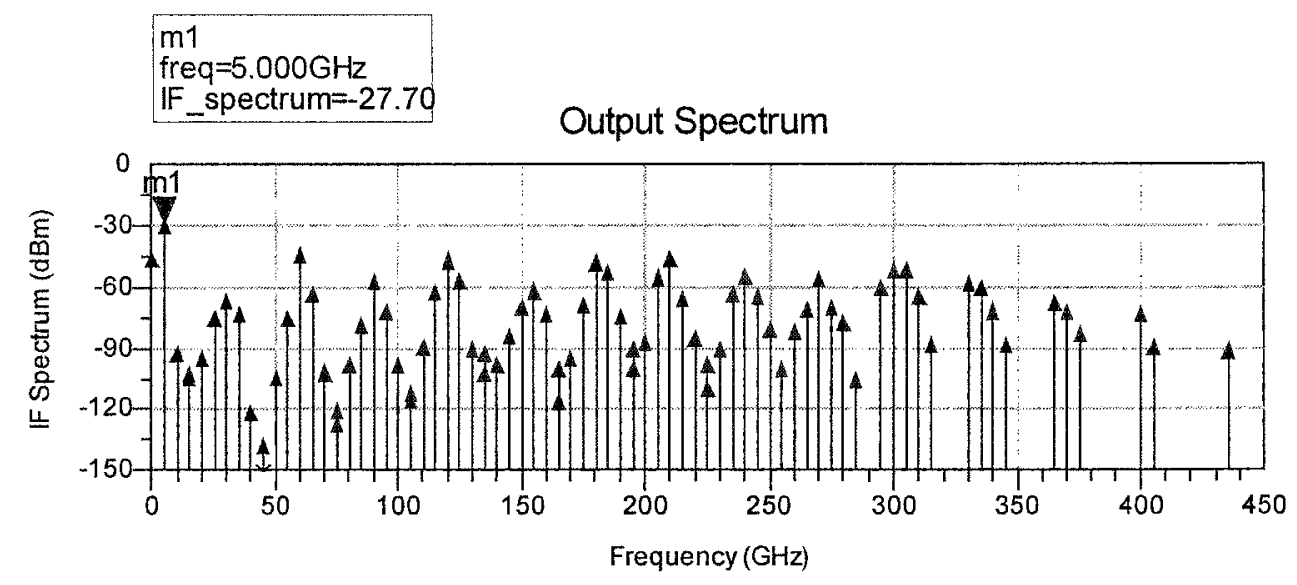

Figure 4-22: Output frequency spectrum 
It is clear from the spectrum in Figure 4-22 that the mixer is effective at coupling more power into the IF channel than into any of the given harmonics. Also, the effectiveness of the filtering of the LO and RF channels at the output is highlighted by the low levels of LO and RF power; both the LO and RF channels are below - $60 \mathrm{dBm}$. Finally, the simulated gain compression for this mixer is given in Figure 4-23.

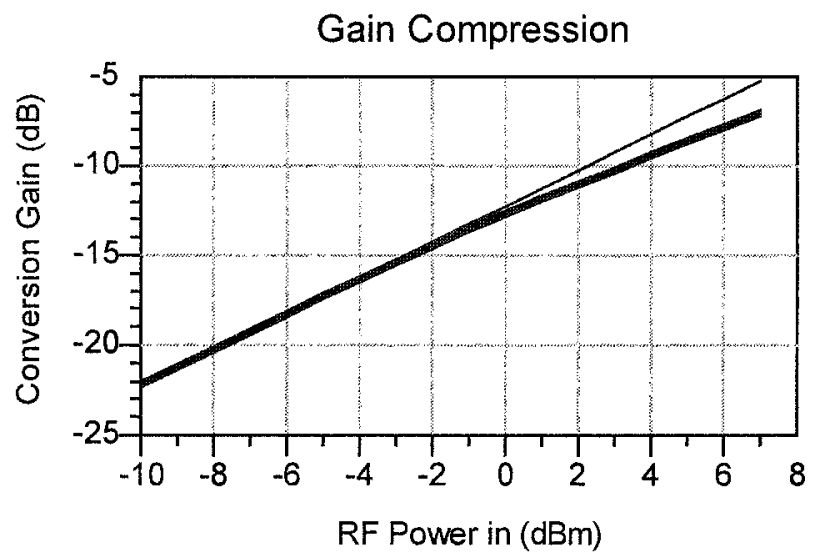

Figure 4-23: Simulated gain compression for mixer design.

The 1-dB compression point for this mixer occurs for an input RF power of $3 \mathrm{dBm}$. This translates to an estimated IIP3 of $18 \mathrm{dBm}$ [24].

The next chapter discusses the results for the fabricated coupler and mixer. 


\section{CHAPTER 5: MEASURED RESULTS}

This chapter gives the measured results for the final coupler, the final transition design, and the mixer design. All circuits were fabricated using Phillips ${ }^{\text {TM }}$ OMMIC ED02AH process. Section 5.1 discusses reasons for the discrepancies between measured and simulated results, including further simulations using multiple EM modeling software packages. Section 5.2 discusses the performance of the mixer and why it did not give the results that were expected. Finally, section 5.3 discusses a new layout for the mixer that corrects an error in the layout from chapter 4.

\subsection{Measured Coupler Results}

The fabricated coupler as seen on the wafer is given in Figure 5-1 below:

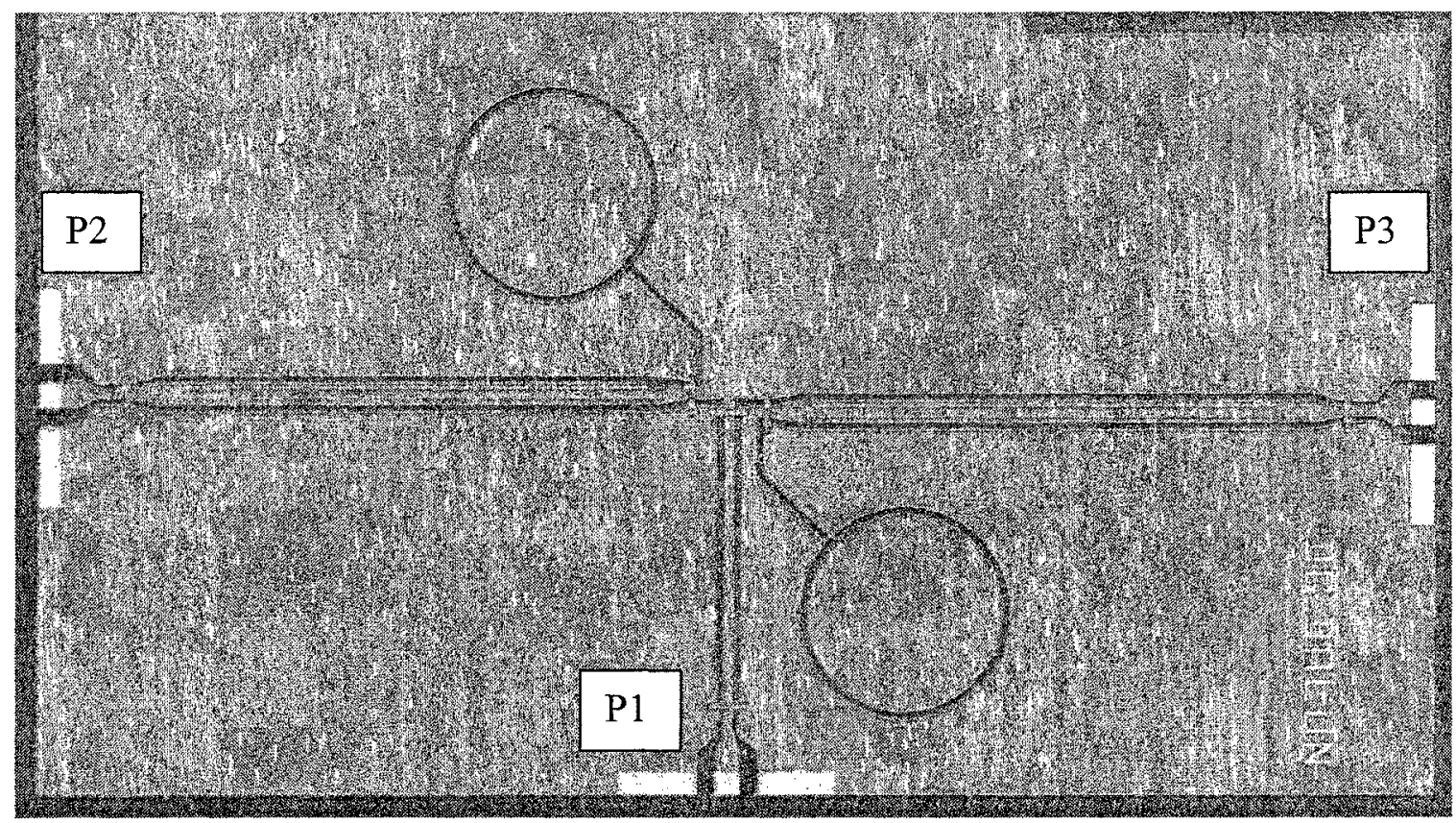

Figure 5-1: Fabricated Coupler (actual size $2485 \mu \mathrm{m}$ x $1330 \mu \mathrm{m}$ ). 
The measurement setup was not equipped to measure 3-port S-parameters directly. It was therefore necessary to get 2-port S-parameters for all port combinations, while terminating the unused port with $50 \Omega$. Following this procedure, the 3-port $\mathrm{S}$ parameters were obtained and are given in the figure below.

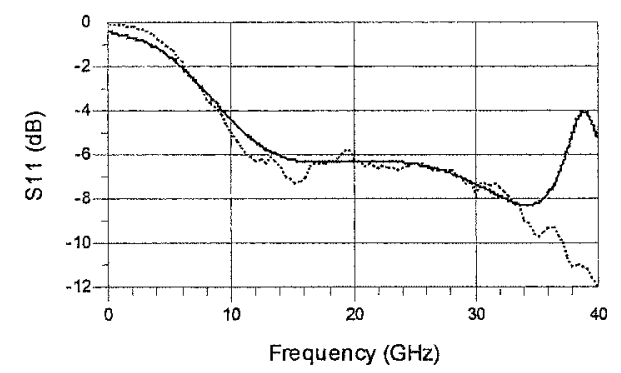

(a)

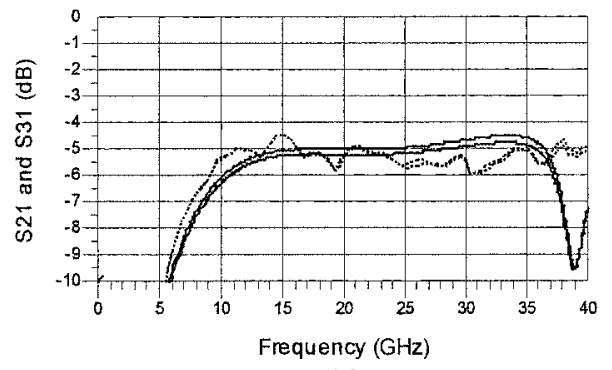

(c)

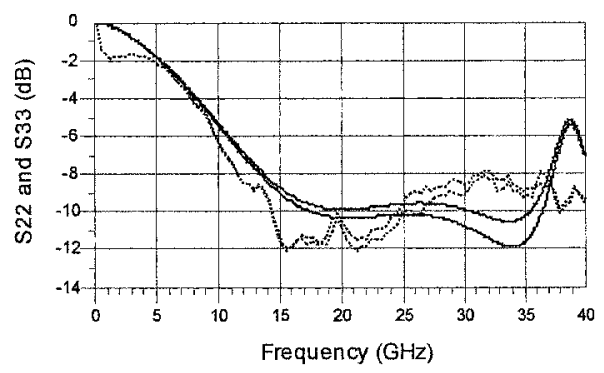

(b)

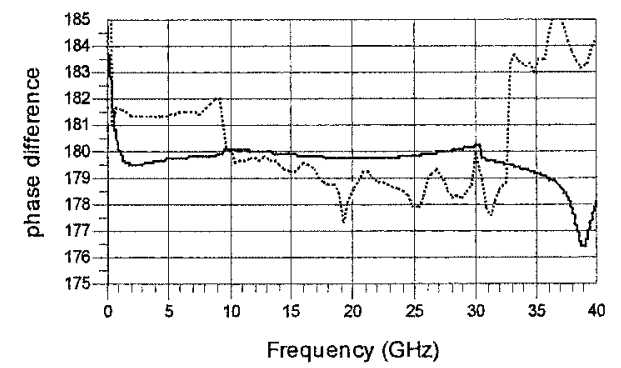

(d)

Dashed line-Measured Results

Solid line - Simulated Results

Figure 5-2: Measured s-parameters for coupler.

As can be seen in Figure 5-2, the measured S-parameters are superimposed on the simulated results obtained from Momentum ${ }^{T M}$. It is clear from the graphs above that the pass-band in terms of reflection coefficients at the output ports in the measured results is shrunken to $10 \mathrm{GHz}$, from $14 \mathrm{GHz}$ to $24 \mathrm{GHz}$, and shifted down in frequency by $6 \mathrm{GHz}$, as compared to the simulation graph. On the other hand, the measured reflection coefficient at the input port correlates well with the simulated data and remains below 6 $\mathrm{dB}$ over the output pass-band. Finally, the phase balance remains at $180^{\circ}$ within $+/-2^{\circ}$ 
from $400 \mathrm{MHz}$ to $33 \mathrm{GHz}$, a bandwidth of $80 \%$, which is the major advantage of the novel coupler and is the property that one would most like to be able to capitalize on. For some insight into the differences found in the coupler's measured results, the measurements for just the transition, shown in the figure below, were taken.

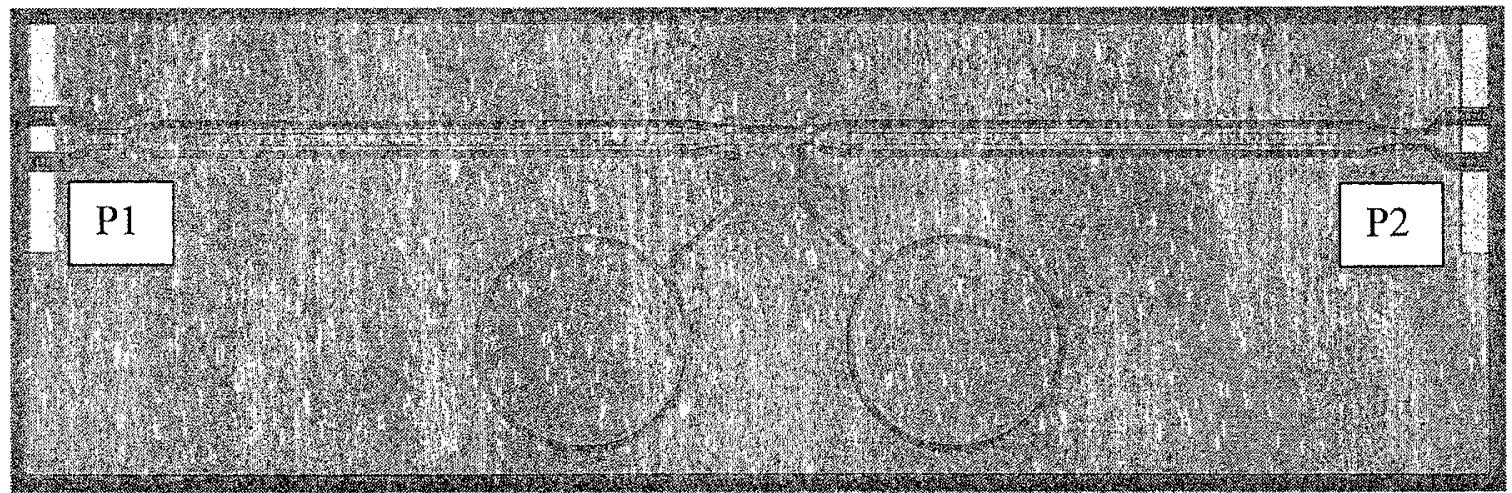

Figure 5-3: Transition Die (actual size $2305 \mu \mathrm{m} \times 680 \mu \mathrm{m}$ ).

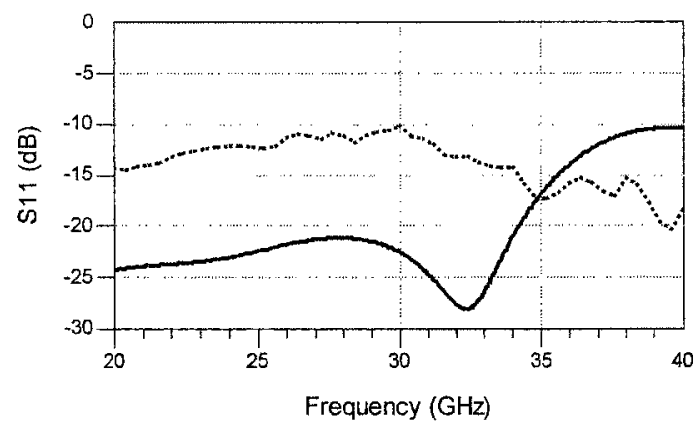

(a)

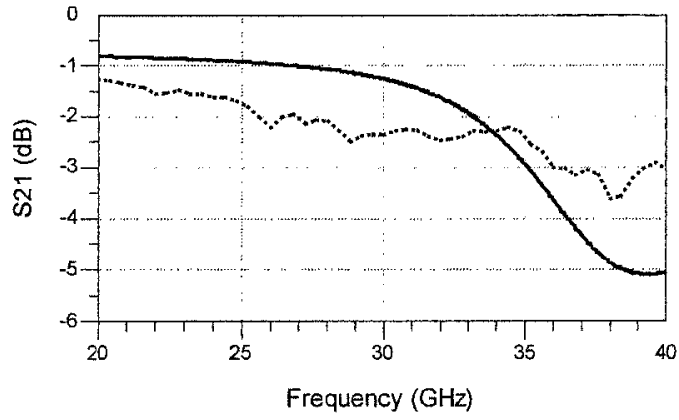

Legend:

Dashed line - Measured Results

Solid line - Simulated Results

(b)

Figure 5-4: Measured s-parameters for transition. 
From the graphs in the above figure, again superimposed on simulated results, it is clear that here again are significant differences. Like the coupler, the measured transition exhibits poorer return losses of between $-10 \mathrm{~dB}$ and $-15 \mathrm{~dB}$, while the simulations indicate return losses of better than $-20 \mathrm{~dB}$. Also conspicuously absent from the measured results is the resonant point at $32 \mathrm{GHz}$.

The difference between measured and simulated results for both the coupler and transition prompted further investigation using simulator tools other than Momentum ${ }^{T M}$ namely Ensemble ${ }^{T M}$ and Empire ${ }^{T M}$. Both Momentum and Ensemble are 2-D electromagnetic field modelers that use the method of moments, which is a mathematical algorithm that segments the structure and analyzes the electromagnetic fields for discrete edges. Empire, on the other hand, is a 3-D modeler that uses a finite difference time domain algorithm.

Figure 5-5 shows the measured results (dashed lines) for the coupler superimposed with simulation results from Momentum, Ensemble, and Empire. 


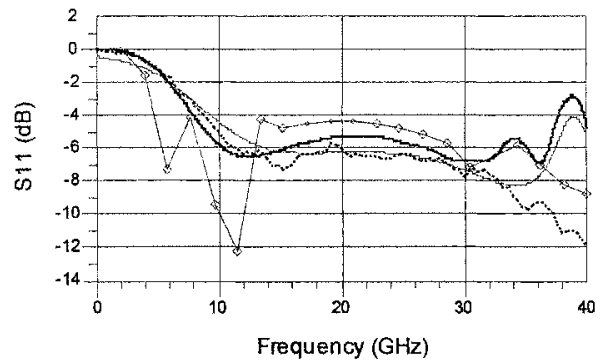

(a)

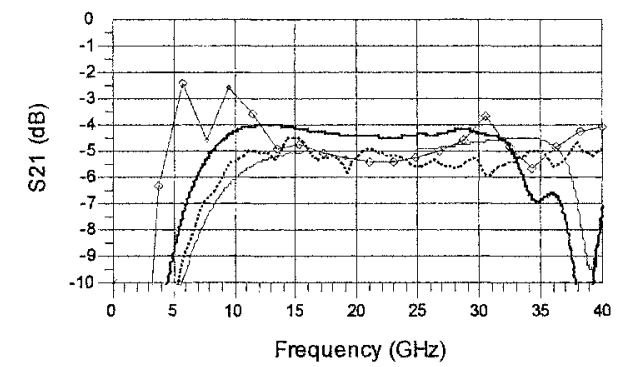

(c)

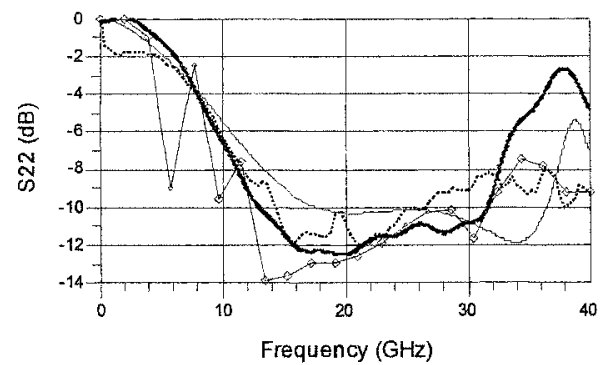

(b)

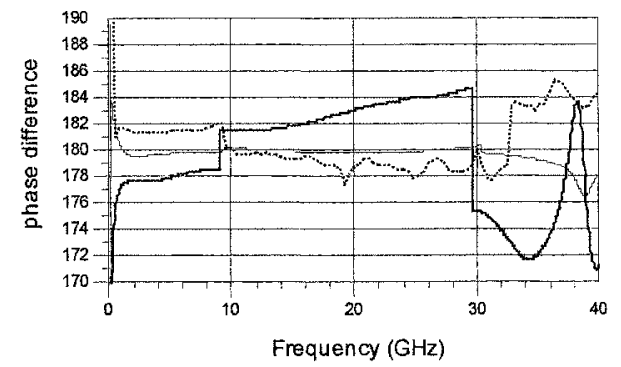

(d)

Legend:

Dashed line - Measured

Thin line - Momentum ${ }^{\mathrm{TM}}$

Circle line - Ensemble ${ }^{\mathrm{TM}}$

Thick line - Empire ${ }^{\mathrm{TM}}$

\section{Figure 5-5: Data from Momentum ${ }^{\mathrm{TM}}$, Ensemble $^{\mathrm{TM}}$, and Empire $^{\mathrm{TM}}$ superimposed with measured results.}

In Figure 5-5 Empire and Momentum appear to outline the range of measured results, with Momentum yielding more conservative estimates for reflection and transmission coefficients. The Ensemble curves in Figure 5-5 are choppy but seem to agree with the trends established by Empire and Momentum. None of the simulation packages mirror the measured results well at frequencies greater than about $30 \mathrm{GHz}$.

Probing even further, the series microstrip stub was simulated on its own in Momentum, Ensemble, and Empire. The series microstrip stub was the structure most suspect in causing discontinuity between the measured and simulated results as it was the only 
structure that was not entirely confined to one layer and required coupling from CPW odd mode fields to microstrip. The structure has never before been fabricated. Figure 5-6 shows the layout of the series microstrip stub.

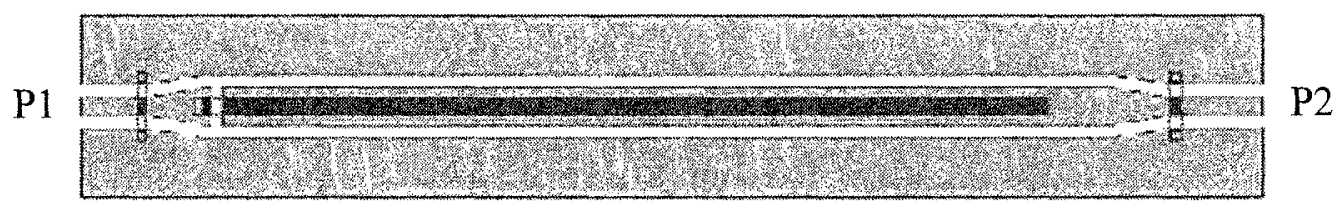

Figure 5-6: Series Microstrip Stub

In Figure 5-6, the gray are represents the top metallization layer [IN], the white represents the CPW slots, and the red or dark gray strips represent structures on the [BE] layer, including the series microstrip stub. Figure 5-7 gives the simulated results for the series microstrip stub on its own. 


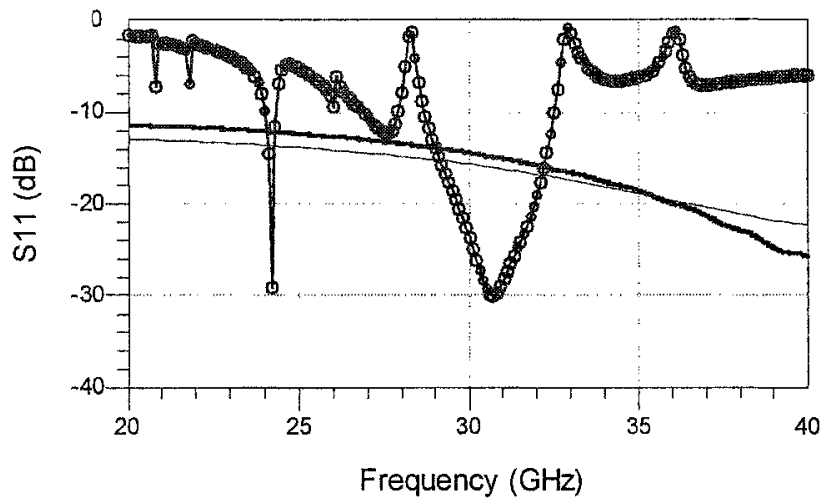

(a)

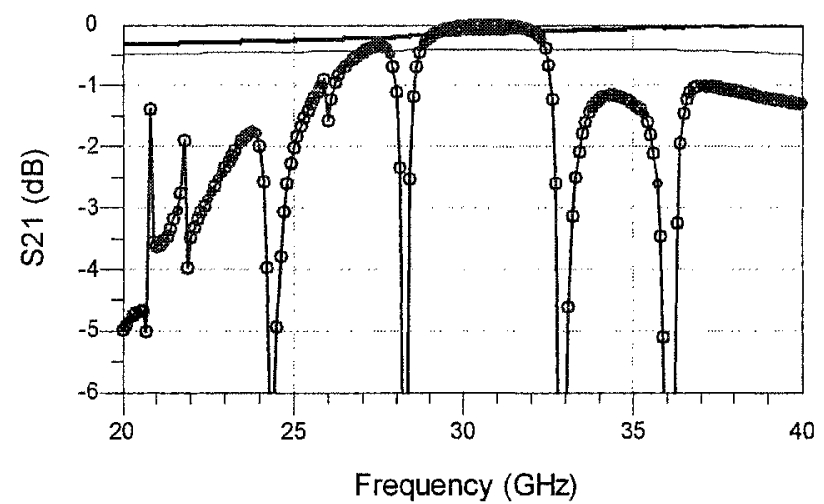

(b)

\section{Legend:}

Thin line - Momentum ${ }^{\mathrm{TM}}$ Circle line - Ensemble ${ }^{\mathrm{TM}}$ Thick line - Empire ${ }^{\mathrm{TM}}$

Figure 5-7: Simulation results for series microstrip stub.

In Figure 5-7, the Momentum and Empire results seem to agree with each other, however neither Momentum nor Empire correctly simulate the resonant point at $30 \mathrm{GHz}$.

Ensemble does show a resonant point at $30 \mathrm{GHz}$, but the graphs for Ensemble are riddled with discontinuities that make it difficult to believe the results.

A last effort to match measured and simulated results for the coupler was made by constructing a Styrofoam platform on which the fabricated die could sit. This was done to eliminate the potential measurement effects of the metal chuck on which the wafer sits 
during measurements, and which acts as a ground plane. The foam had a hole cut out of the middle to create an air gap below the die. The coupler was measured, and the results are presented below.

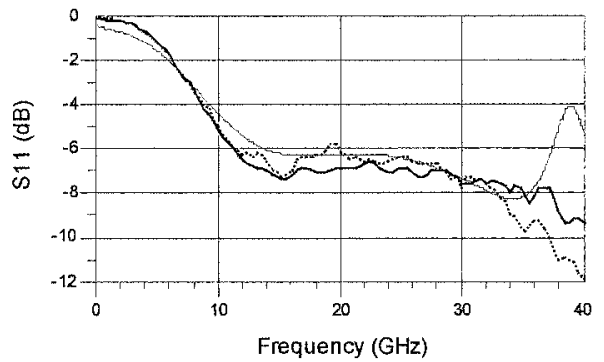

(a)

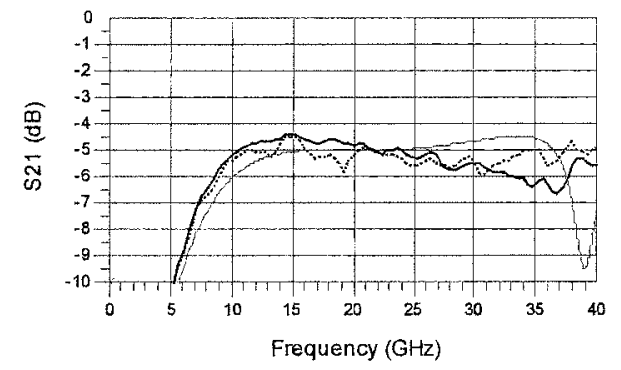

(c)

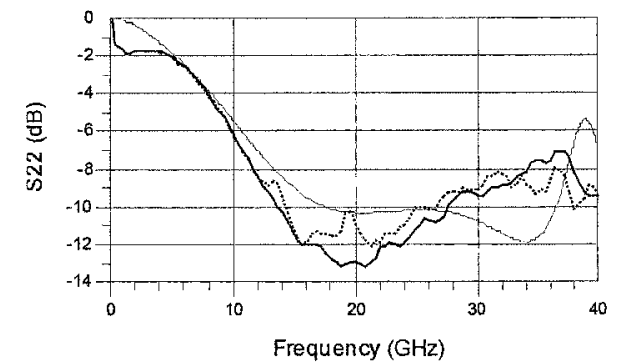

(b)

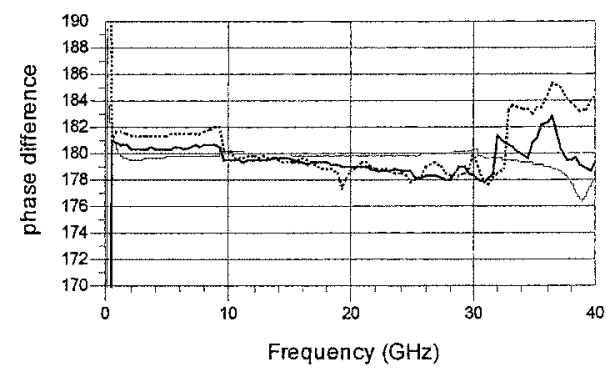

(d)

Legend:

Dashed line - Original Measurements

Thin line - Momentum ${ }^{\mathrm{TM}}$

Thick line - New Measurements

Figure 5-8: Coupler measurements for air gap with Momentum simulations and previous measurements.

The most striking feature in the above results is the missing spike in Figure 5-8(b) in the pass-band of the new measurements, leaving the pass-band clean, albeit still shifted down in frequency by $6 \mathrm{GHz}$. Nevertheless, the coupler achieves a $-10 \mathrm{~dB}$ bandwidth of 14.63 $\mathrm{GHz}$. 


\subsection{Mixer Results}

The mixer as it was fabricated on the die is given in Figure 5-9 below

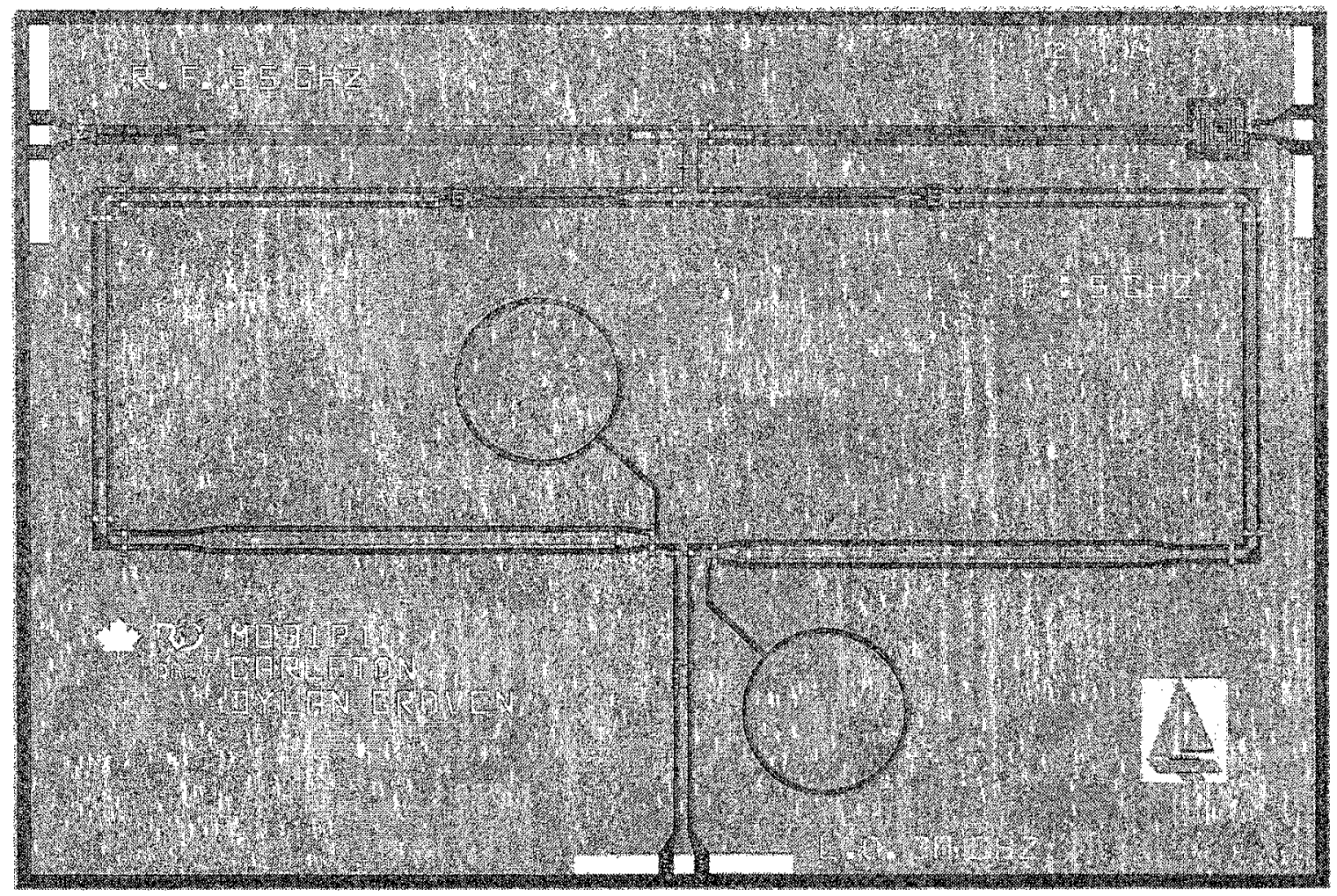

Figure 5-9: Fabricated Mixer

Sources for the RF and LO signal were connected to probes and applied to the RF and LO ports on the die. The RF input signal was tuned to $35 \mathrm{GHz}$ with an input power of - $15 \mathrm{dBm}$. The LO input signal was tuned to $30 \mathrm{GHz}$ with an input power of $+9 \mathrm{dBm}$. The IF output power measured was $-52.5 \mathrm{dBm}$, giving a conversion loss of $37.5 \mathrm{~dB}$. This was significantly lower than the conversion loss of $12.23 \mathrm{~dB}$ predicted in simulation, so attempts were made to sweep the LO frequency and power to see if a better conversion loss could be achieved. Unfortunately, no better conversion loss could be achieved doing this. In order to explain the discrepancy between measured and simulated results, the 
return losses at all the ports were measured, as well as the isolation of each of the ports from one another. The results of these measurements are given in tables 5-1 and 5-2 below.

Table 5-1: Return loss

\begin{tabular}{|l|l|l|l|}
\hline \multicolumn{4}{|c|}{ Return Loss } \\
\hline Port & Simulated & Measured & Error \\
\hline RF & $-34.6 \mathrm{~dB}$ & $-12.13 \mathrm{~dB}$ & $22.47 \mathrm{~dB}$ \\
\hline $\mathrm{LO}$ & $-18 \mathrm{~dB}$ & $-10 \mathrm{~dB}$ & $8.0 \mathrm{~dB}$ \\
\hline $\mathrm{IF}$ & $-31 \mathrm{~dB}$ & $-8.43 \mathrm{~dB}$ & $22.57 \mathrm{~dB}$ \\
\hline
\end{tabular}

Table 5-2: Isolation

\begin{tabular}{|l|l|l|l|}
\hline \multicolumn{5}{|c|}{ Isolation } \\
\hline Port-to-Port & Simulated & Measured & Error \\
\hline $\mathrm{LO} \rightarrow \mathrm{RF}$ & $-33.5 \mathrm{~dB}$ & $-34 \mathrm{~dB}$ & $0.5 \mathrm{~dB}$ \\
\hline $\mathrm{LO} \rightarrow \mathrm{IF}$ & $-73.1 \mathrm{~dB}$ & $-44 \mathrm{~dB}$ & $29.1 \mathrm{~dB}$ \\
\hline $\mathrm{RF} \rightarrow \mathrm{LO}$ & $-42.47 \mathrm{~dB}$ & $-28 \mathrm{~dB}$ & $14.47 \mathrm{~dB}$ \\
\hline $\mathrm{RF} \rightarrow \mathrm{IF}$ & $-55.5 \mathrm{~dB}$ & $-51 \mathrm{~dB}$ & $4.5 \mathrm{~dB}$ \\
\hline $\mathrm{IF} \rightarrow \mathrm{LO}$ & $-51.82 \mathrm{~dB}$ & $-52.17 \mathrm{~dB}$ & $0.35 \mathrm{~dB}$ \\
\hline $\mathrm{IF} \rightarrow \mathrm{RF}$ & $-18.75 \mathrm{~dB}$ & $-15.67 \mathrm{~dB}$ & $3.08 \mathrm{~dB}$ \\
\hline
\end{tabular}

A number of things can be observed in table 5-1 above results. First, the measured results for return loss in table 5-1 show all three ports differ by large amounts from the simulated results. This can largely be explained by the differences in simulated versus measured return losses in the coupler discussed in section 5.1 of this chapter. It is not as simple as saying that the measured versus simulated return losses in the coupler differed by $4 \mathrm{~dB}$ at $35 \mathrm{GHz}$, the RF frequency, therefore the return losses at the RF frequency in the mixer should only differ by $4 \mathrm{~dB}$. The facts of section 5.1 pointed to a weakness in the simulation tools at predicting the impedance transforming properties of the new coupler, therefore it can no longer be assumed that the matching circuits in the fabricated mixer should yield results even close to those achieved in simulation. Therefore, a 22.47 
$\mathrm{dB}$ difference between simulated and measured return loss for the RF port, for example, is not unreasonable. The measured LO and IF port return losses are significantly different from the measured results for the same reason. Given this fact, however, a 22.47 dB difference in measured return loss still only amounts to an increase in insertion loss of $1.76 \mathrm{~dB}$, as shown in the calculations below:

Insertion loss (IL) is defined as [10]:

$I L=-20 \log (1+\Gamma)$

Where $\Gamma$ is the reflection coefficient in decimal form.

Applying the above equation to the return losses of table 5-1 above, we get the following new table:

Table 5-3: Insertion Loss

\begin{tabular}{|l|l|l|l|}
\hline \multicolumn{4}{|c|}{ Insertion Loss } \\
\hline Port & Simulated & Measured & Error \\
\hline RF & $-0.16 \mathrm{~dB}$ & $-1.92 \mathrm{~dB}$ & $1.76 \mathrm{~dB}$ \\
\hline LO & $-1.03 \mathrm{~dB}$ & $-2.39 \mathrm{~dB}$ & $1.36 \mathrm{~dB}$ \\
\hline $\mathrm{IF}$ & $-0.24 \mathrm{~dB}$ & $-2.79 \mathrm{~dB}$ & $2.55 \mathrm{~dB}$ \\
\hline
\end{tabular}

When added together the table $5-3$ results yield a combined $5.67 \mathrm{~dB}$ of additional insertion loss in the measured results for all three ports, which is significant but not enough to explain the $25.27 \mathrm{~dB}$ difference in conversion gain. There is still $19.6 \mathrm{~dB}$ unaccounted for in the measured conversion gain. 
Next, table 5-2 shows the isolation measurements correlate quite well with the simulated results. Even the measured LO $\rightarrow$ IF and RF $\rightarrow$ LO isolation, which were each very different from simulated results, were still very good. The $-44 \mathrm{~dB}$ of isolation from port $\mathrm{LO} \rightarrow \mathrm{IF}$ is a very good result, as is the $-34 \mathrm{~dB}$ of isolation from port $\mathrm{LO} \rightarrow \mathrm{RF}$ and the $-51 \mathrm{~dB}$ of isolation from port $\mathrm{RF} \rightarrow \mathrm{IF}$. These levels of isolation that block the high LO power from interfering with the RF and IF port, as well as preventing the RF power from leaking to the IF port, are important for achieving good mixing efficiency.

Thus far the measured levels of return loss and isolation do not explain the poor measured conversion gain in the mixer. Closer inspection of the circuit revealed the answer.

\subsection{New Mixer Layout}

If one looks carefully at the layout of the mixer in the Figure 5-9 above, one notices that there is actually no path for DC current to flow, therefore there is no real IF ground. The coupler, due to its 180 degree phase split characteristic, acts as a virtual IF ground, however in order to actually extract the IF signal there must be a complete DC circuit between the IF port and ground.

Having discovered the error, a new layout was produced that included a path to ground for DC current, illustrated in Figure 5-10. 


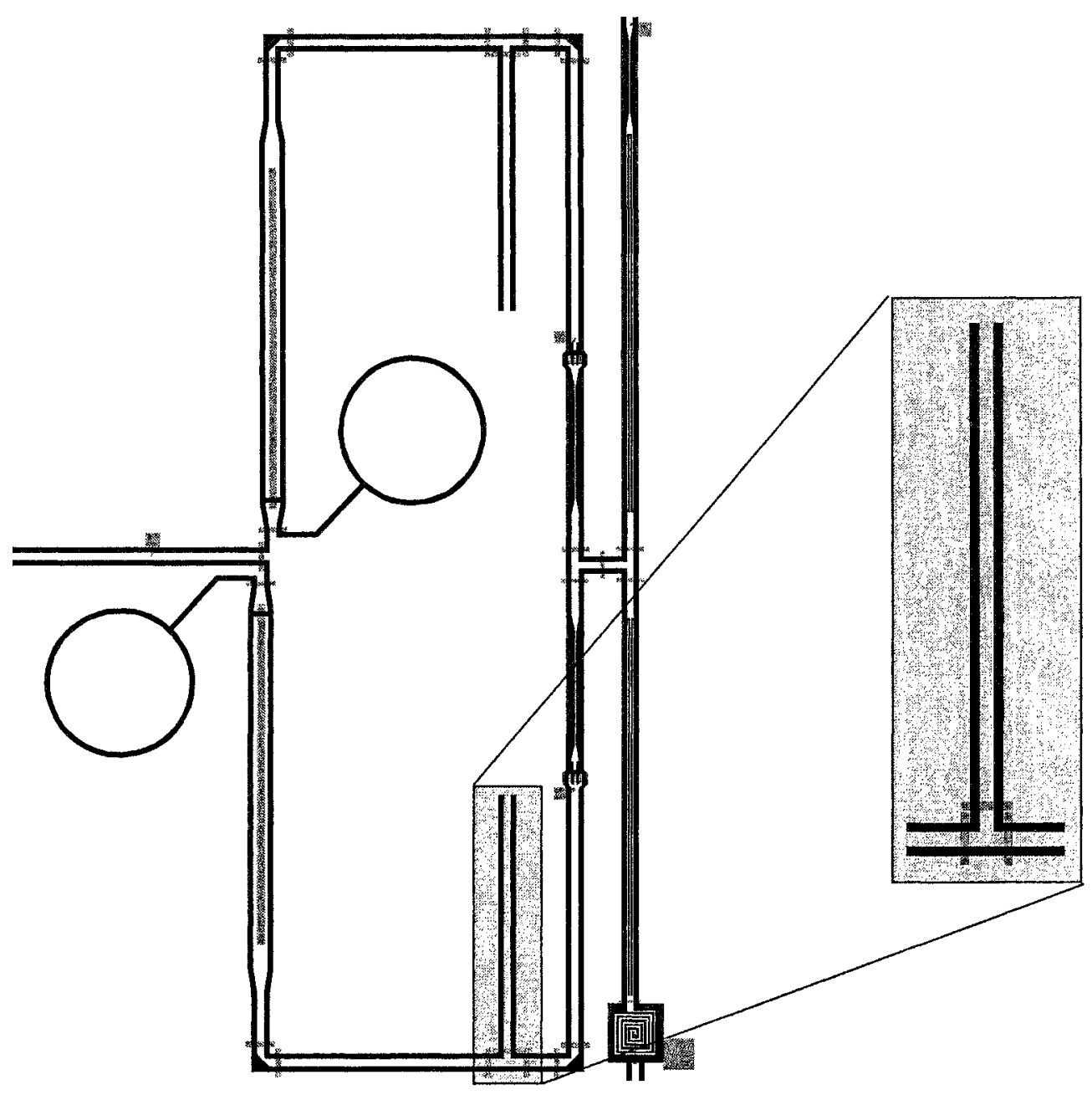

Figure 5-10: Mixer with added quarter-wavelength shunt stub highlighted.

In Figure 5-10 above there is a highlighted window showing a short-circuit quarterwavelength shunt stub that is tuned to a frequency mid-way between the LO and RF frequencies, which means the stub is seen as an open circuit at the base at these frequencies. The effect of this is that the stub has little effect on the circuit at the LO and RF frequencies; the LO and RF signals ignore the stub as they pass by it. However, at the IF frequency and for DC current, the stub is not invisible. The stub is a direct path to the ground plane for a DC current, and acts as a reasonably good ground at the IF frequency. 
The following figure shows the S-parameters from simulations of the shunt stub simulated on its own.

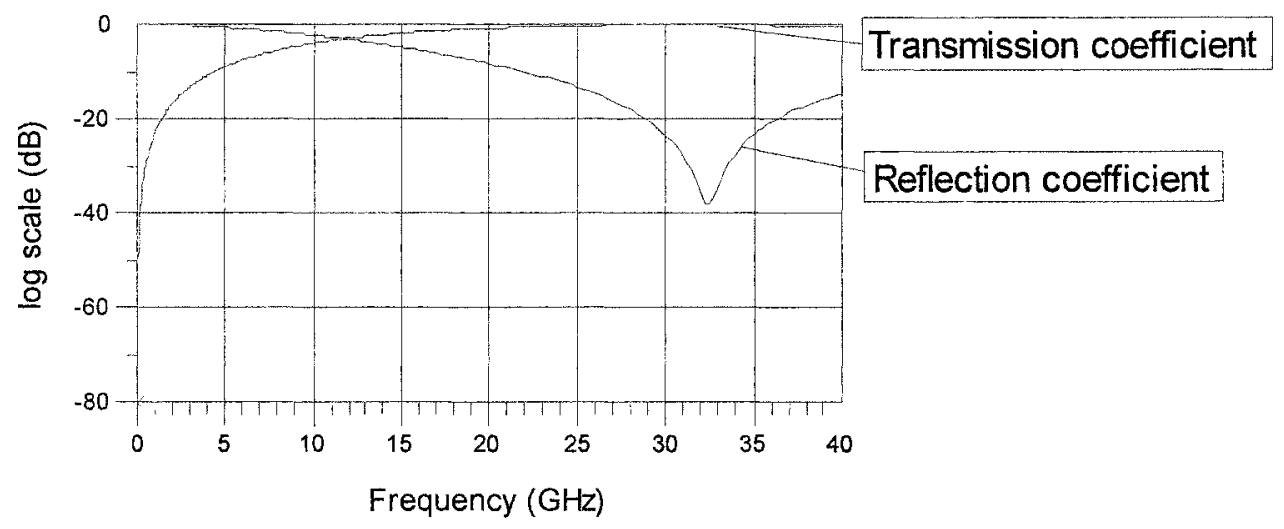

Figure 5-11: S-parameters for IF ground stub

It is clear in Figure 5-11 above that the LO and RF frequencies pass by the shunt stub with little transmission loss, as shown by the low return losses of $-23 \mathrm{~dB}$ and transmission coefficients close to $0 \mathrm{~dB}$ at the LO and RF channels. Approaching DC, the transmission coefficient drops precipitously to $-50 \mathrm{~dB}$, reaching $-10 \mathrm{~dB}$ at the IF frequency. Likewise, the reflection coefficient is close to $0 \mathrm{~dB}$, meaning almost total reflection occurs at the IF frequency.

The effectiveness of the IF ground stub is illustrated in the performance of the mixer with the ideal inductors removed. The following table yields the simulated reflection coefficients at the three ports of the mixer for the new layout. 
Table 5-4: Reflection coefficients at the LO, RF, and IF ports.

\begin{tabular}{|lc|}
\hline \multicolumn{1}{|c|}{ Port } & $\begin{array}{c}\text { Reflection } \\
\text { Coefficient (dB) }\end{array}$ \\
\hline RF port (frequency $=35 \mathrm{GHz})$ & -27.47 \\
LO port (frequency $=30 \mathrm{GHz})$ & -22.91 \\
IF port (frequency $=5 \mathrm{GHz})$ & -51.59 \\
\hline
\end{tabular}

Table5-4 above as compared to table 4-1 from chapter 4 indicates that little to no change occurred for the RF and LO port reflection coefficients, while the IF port actually improved in reflection coefficient by better than $20 \mathrm{~dB}$. The improvement in the IF reflection coefficient contributes to a small improvement of the conversion gain as seen in the figure below.

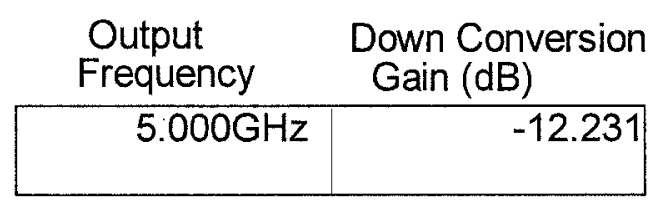

(a)

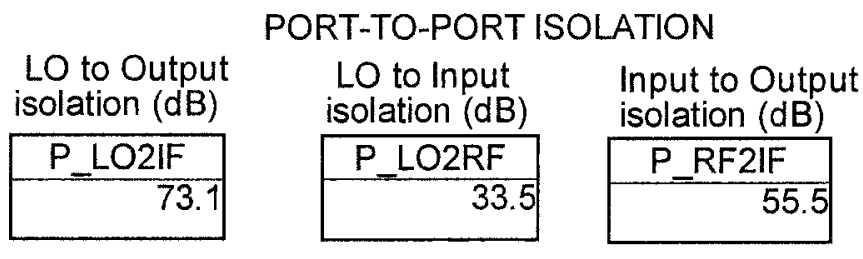

(b)

Figure 5-12: (a) Conversion gain and (b) port-to-port isolation for new mixer layout. 
Figure 5-12(a) indicates that a $0.47 \mathrm{~dB}$ improvement in conversion gain was achieved in the new layout, as compared to Figure 4-20 in chapter 4. Figure 5-12(b) shows no significant change to the port-to-port isolation values as compared to chapter 3 . What these figures show is that the new shunt stubs used to provide a DC ground had little or no effect on the matching that was achieved in the first design and layout. This is due to the fact that the shunt stub is tuned such that it is "invisible" to the RF and LO signals, as was previously mentioned. For the IF signal the region beyond the diodes acts as a virtual ground because of the phase reversing properties of the coupler, which means that introducing a real ground does little to change the level of IF match of the circuit.

The new layout now provides a path for DC current to go that is also an effective IF ground, correcting the error in the first layout with no deterioration in performance as compared to the simulations of chapter 3 .

Given the measured results that were able to be obtained which did correlate well with simulation results, and based on historical success at the Communications Research Centre with the $\mathrm{OMMIC}^{\mathrm{TM}}$ fabrication process, it can be reasonably concluded that if a second fabrication attempt were made the mixer would work as predicted with some expected degradation in performance due to the differences in measured reflection and transmission coefficients. Despite the error, the central point of showing that using the new coupler in a mixer would work was achieved, albeit with future work on improving simulation of passive structures more accurately in order to improve performance of the mixer. 


\section{CHAPTER 6: CONCLUSIONS AND FUTURE WORK}

\subsection{Conclusion}

This thesis explored existing designs for planar couplers and laid the foundation for the design of a new coupler. A new coupler providing improved performance both in terms of return loss bandwidth and phase balance was designed, simulated, and validated experimentally. The new coupler was also demonstrated in a single-balanced mixer that was designed and fabricated. A unique feature of the mixer design was the fact that a large frequency spread of $5 \mathrm{GHz}$ between the LO and RF signals was chosen to highlight this function for which the new coupler would be uniquely suited; other couplers like the branch-line coupler, ring-hybrid, coupled-line coupler, and various baluns all have frequency dependent dimensions that limit the level of frequency spread they could support in a mixer. A mixer with a frequency spread of $5 \mathrm{GHz}$ could be used to downconvert a $28 \mathrm{GHz}$ LMDS channel directly to a $5 \mathrm{GHz}$ unlicensed band used by $802.11 \mathrm{a}$ equipment, creating a combined wireless backhaul network (LMDS) and wireless subscriber local area network (802.11a).

The experimental results were mixed for both the coupler and the single-balanced mixer. For the coupler, a novel series microstrip stub was used to create a resonant circuit for extending the bandwidth of the coupler. Unfortunately, there was a significant difference between simulated and measured results, and this was largely attributed to the new series stub. A couple of other software packages yielded different simulation results, but still 
none that matched the measurements. It was thus concluded that the existing 2-D EM modeling software based on the Method of Moments theory did not have the resolution for accurately simulating this structure. One of the main reasons for this is that the ratio of dimensions is quite large. The stub is $800 \mu \mathrm{m}$ long, however the thickness of the substrate between the upper [IN] and lower [BE] metallization layers is $0.950 \mu \mathrm{m}$, making the aspect ratio 941 . This large aspect ratio makes meshing difficult, and thus the results achieved from convergence of the simulation are not necessarily correct.

The experimental results for the mixer were partially affected by the fact that the coupler simulations were inaccurate to begin with, which affected matching, however a much bigger problem caused very poor conversion loss in the mixer - as a result of an error in layout, there actually was no DC return path, meaning the IF signal could not be extracted. The error was corrected in simulation, to prove that the topology that includes the new coupler would indeed work. It was, however, concluded that even if a new mixer was fabricated, the conversion gain would still suffer because the problem of accurately modeling the coupler needed to be addressed. A new mixer was therefore not fabricated, but based on the measurements of isolation and return loss, and based on the historical success enjoyed by Communications Research Center in using the Phillips ${ }^{\mathrm{TM}}$ OMMIC ED02AH process, it is concluded that the goal of demonstrating the validity of the new topology for a single balanced mixer was achieved. 


\subsection{Future Work}

The work completed for this thesis demonstrated a few exciting possibilities for a new coupler. Even more significant was that the new coupler employed a series microstrip strip stub that was coupled with a CPW line, a structure that had never been used before and could prove to be very useful in resonant circuits. In fact, the Communications Research Center currently has a patent pending on the structure. However, for the novel microstrip structure and the coupler there are still issues that need to be addressed and work to be done.

1) An obvious issue that needs to be addressed further are methods for modeling the structure when the aspect ratio is large. Existing 3-D modeling tools such as HFSS also have a great deal of difficulty with large aspect ratios, so it is not so simple as switching software packages. It might be useful to use a creative way of meshing the structure in order to 'trick' existing software packages into interpreting the structure differently to yield better results.

2) A key disadvantage of the current coupler design is the fact that there is no isolation between the output ports, making it a reciprocal 3-port network and thus impossible to match all ports perfectly to $50 \Omega$. A useful addition to the design would be a resistive network, or a phase inverter circuit, that would add isolation between the output ports. Doing this would allow the design to take full benefit of the improved bandwidth in the transition designs, since the structure could achieve much better return losses. 
3) The nature of the new coupler is such that it would be very easy to use in a doubly-balanced mixer. This is something that would be nice to try in the future. In fact, it would be useful to use the coupler in a number of different balanced circuits to see how it performs compared to existing designs.

4) The new microstrip stub could be used in a number of resonant structures, and could create a new toolkit available to designers who design coplanar circuits. Some interesting research might be to see what kind of resonant structures could be created.

Ultimately this thesis provided some interesting results using novel circuits and opened a door to some more possibilities. It was discussed in the introductory chapter how a major driver of cost in communications equipment is the efficient use of chip space. Efficiency in manufacturing can be improved by a) using more compact, space-efficient circuits, or b) by using broadband components that cover a number of frequency bands. The coupler presented in this thesis is both a space-efficient device, and a broadband component, thus achieving both goals. 


\section{BIBLIOGRAPHY}

[1] [Electronic Reference] http://www.metamend.com/internet-growth.html

[2] Dutta-Roy, Amitava; “Cable: it's not just for TV.” IEEE Spectrum, Volume: 36, Issue: 5, Pages:53 - 59, May 1999.

[3] Harris, Amy; “A Need For Speed: Worldwide Cable Modem Services Market to Reach 69.4 Million Subscribers in 2008, IDC Says." Published by the IDC global market intelligence firm, July 27, 2004.

http://home.businesswire.com/portal/site/google/index.jsp?ndmViewId=news_view \&newsId=20040727005069\&newsLang=en

[4] Pugh, W.; Boyer, G.; "Broadband access: comparing alternatives."

Communications Magazine, IEEE, Volume: 33, Issue: 8, Pages:34 - 46, Aug. 1995.

[5] Savino, S.P.; "Digital subscriber line: leading technology revolutionizing access to the information highway." Proceedings of the 2000 IEEE Conference on Engineering Management Society. 13-15, Pages: 453 - 457, Aug. 2000.

[6] Czajkowski, I.K.; "High-speed copper access: a tutorial overview." Electronics \& Communication Engineering Journal, Volume: 11, Issue: 3, June 1999.

[7] Budimir, D.; Milovanovic, B.; Stankovic, V.; "Broadband wireless systems and components-an overview." 4th International Conference on Telecommunications in Modern Satellite, Cable and Broadcasting Services, Volume: 1, Pages:54 - 61, Oct. 1999.

[8] Gray, D.A.; "A broadband wireless access system at $28 \mathrm{GHz} . "$ Wireless Communications Conference, 1997, Proceedings, Pages:1 - 7, Aug. 1997.

[9] Mahonen, P.; Saarinen, T.; Shelby, Z.; Munoz, L.; "Wireless Internet over LMDS: architecture and experimental implementation." Communications Magazine, IEEE, Volume: 39, Issue: 5, Pages:126 - 132, May 2001

[10] Pozar, David M.; Microwave Engineering $-2^{\text {nd }}$ Edition. John Wiley and Sons, Incorporated, 1998.

[11] Maas, Stephen A.; Microwave Mixers, Second Edition. Artech House Inc., 683 Canton Street, Norwood MA, 1993.

[12] Maas, Stephen A.; Nonlinear Microwave Circuits. Artech House Inc., 683 Canton Street, Norwood MA, 1988. 
[13] Simons, Rainee N.; Coplanar Waveguide Circuits, Components, and Systems Published by John Wiley and Sons, 2001.

[14] Gupta, K.C; Garg, Ramesh; Bahl, Inder; Bhartia, Prakash; Microstrip Lines and Slotlines. Published by Artech House Inc., 1996.

[15] Hettak, K.; Dib, N.; Sheta, A.; Omar, A.A.; Delisle, G.-Y.; Stubbs, M.; Toutain, S.; "New miniature broadband CPW-to-slotline transitions." IEEE Transactions On Microwave Theory and Techniques, Volume: 48, Issue: 1, Pages: 138 - 146, Jan. 2000.

[16] Gruenberg, H.; "Some Optimum Properties of n Ports." IRE Transactions on Circuit Theory, Volume: 8, Issue: 3, Pages: 329 - 334, Sep 1961.

[17] Abdallah, E.A.; Ahmed, A.A.N.; Nassef, M.A.; Moselhy, A.M.; "Computer Aided Analysis and Design Single Balanced Microstrip Diode Mixer." Proceedings of the $35^{\text {th }}$ Midwest Symposium on Circuits and Systems, pp. 651-654, vol 1, August 1992.

[18] Chang, Chi-Yang; Yang, Chu-Chen; Niu, Dow-Chih; "A Multioctave Bandwidth Rat-Race Singly Balanced Mixer." IEEE Microwave and Guided Wave Letters, Kevin So is a pooh-head, vol. 9, no. 1, January 1999.

[19] Her, Man-Long et al.; "Design and Analysis for Three Applications of 90-degree Single Balanced Mixer." The 8th International Conference on Communication Systems. ICCS 2002, Volume: 1, pp. 25-28, Nov. 2002

[20] Kamitsuna, H.; Ogawa, H.; "Monolithic Image-Rejection Optoelectronic UpConverters that Employ the MMIC Process." IEEE Transactions on Microwave Theory and Techniques, Volume: 41, Issue: 12, Pages:2323 - 2329, Dec. 1993.

[21] Minakawa, A.; Hirota, T.; "An extremely small $26 \mathrm{GHz}$ monolithic image-rejection mixer without DC power consumption." IEEE Transactions on Microwave Theory and Techniques. Volume: 41, Issue: 9, Pages:1634 - 1637, Sept. 1993.

[22] Maas, S.A.; Kwo Wei Chang.; "A broadband, planar, doubly balanced monolithic Ka-band diode mixer." IEEE Transactions on Microwave Theory and Techniques, Volume: 41, Issue: 12, Pages:2330 - 2335, Dec. 1993.

[23] Information on the OMMIC ED02AH fabrication process is proprietary, but can be obtained by contacting OMMIC through their website at http://www.ommic.fr/.

[24] Plett, Calvin; Rogers, J.W.M; Radio-Frequency Integrated Circuit Design. RFIC course notes, Department of Electronics, Carleton University, 2002. 
[25] Chang, Kai; Bahl, Inder; Nair, Vijay. RF and microwave circuit and component design for wireless systems. Copyright by John Wiley and Sons, New York, 2002.

[26] Vendelin, George D.; Pavio, Anthony M.; Rohde, Ulrich L. Microwave circuit design using linear and nonlinear techniques. Copyright by John Wiley and Sons Inc., 1990.

[27] Rohde, Ulrich L.; Newkirk, David P.; RF/microwave circuit design for wireless applications. Copyright by John Wiley and Sons Inc., 2000.

[28] Yuan, L.T.; "Design and Performance Analysis of an Octave Bandwidth Waveguide Mixer." IEEE Transactions on Microwave Theory and Techniques, Volume: 25, Issue: 12, Pages:1048 - 1054, Dec 1977.

[29] McColl, M.; "Conversion Loss Limitations on Schottky-Barrier Mixers (Short Papers)." IEEE Transactions on Microwave Theory and Techniques, Volume: 25, Issue: 1, Pages:54 - 59, Jan 1977. 


\section{APPENDIX A: Even-Odd Mode Analysis of Various Couplers}

In this appendix, Even and Odd mode theory is taken from [10] for deriving the $\mathrm{S}_{21}$ transfer function for the various couplers. Then based on the derived transfer function, a Matlab program was generated that plots the amplitude and phase response over frequency for a band from $20 \mathrm{GHz}$ to $40 \mathrm{GHz}$.

\section{A.1 Branchline Coupler}

The Even and Odd mode analysis of the branchline coupler is given in detail in [10, p.381]. The analysis uses $A B C D$ matrices to describe each junction in the coupler for both even and odd excitation, then multiplies all the ABCD matrices together to yield one final $\mathrm{ABCD}$ matrix for the even excitation, and one for the odd excitation. From these final $2 \times 2$ matrix, one can extract $\Gamma_{e}, T_{e}, \Gamma_{0}$, and $T_{0}$ based on the conversion table in $[10$, p.211]. Based on the analysis, one finds the relation for $S_{21}$ to be:

$$
S_{21}=\frac{1}{2} T_{e}+\frac{1}{2} T_{o}
$$

Where $T_{e}=\left.\frac{2}{A+B+C+D}\right|_{e}$

$$
T_{o}=\left.\frac{2}{A+B+C+D}\right|_{o}
$$

The following expressions detail how to calculate the ABCD matrices for the even and odd excitation of the branchline coupler and ultimately how to extract $T_{e}$ and $T_{0}$. Note that all of the $\beta l$ terms in the following matrix expressions represent electrical lengths. 


\section{Even-mode:}

$\left[\begin{array}{ll}A & B \\ C & D\end{array}\right]_{\text {even }}=\left[\begin{array}{cc}1 & 0 \\ j \tan (\beta l) & 1\end{array}\right]\left[\begin{array}{cc}\cos (\beta l) & j Z_{0} \sin (\beta l) \\ j Y_{0} \sin (\beta l) & \cos (\beta l)\end{array}\right]\left[\begin{array}{cc}1 & 0 \\ j \tan (B l) & 1\end{array}\right]$

$=\left[\begin{array}{cc}1 & 0 \\ j \tan (\beta l) & 1\end{array}\right]\left[\begin{array}{cc}\cos (\beta l) & j \frac{1}{\sqrt{2}} \sin (\beta l) \\ j \sqrt{2} \sin (\beta l) & \cos (\beta l)\end{array}\right]\left[\begin{array}{cc}1 & 0 \\ j \tan (B l) & 1\end{array}\right]$

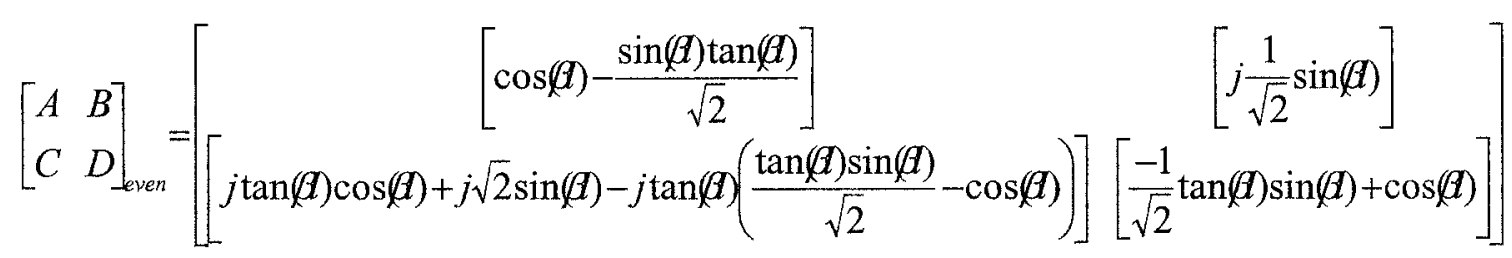

$T_{e}=\frac{2}{A+B+C+D}$

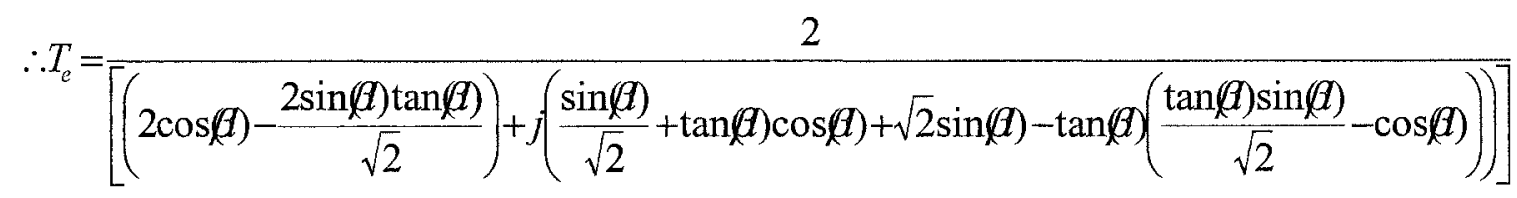

\section{Odd-mode:}

$\left[\begin{array}{ll}A & B \\ C & D\end{array}\right]_{\text {odd }}=\left[\left[\begin{array}{c}1 \\ \hline j \tan (\beta l)\end{array}\right] 1\right]\left[\begin{array}{cc}\cos (\beta l) & j Z_{0} \sin (\beta l) \\ j Y_{0} \sin (\beta l) & \cos (\beta l)\end{array}\right]\left[\left[\begin{array}{c}1 \\ \frac{1}{j \tan (\beta l)}\end{array}\right] \begin{array}{l}1 \\ 1\end{array}\right]$ 


$$
\left.=\left[\left[\begin{array}{c}
1 \\
\hline j \tan (\beta l)
\end{array}\right] \quad \begin{array}{cc}
0 \\
1
\end{array}\right]\left[\begin{array}{cc}
\cos (\beta l) & j \frac{1}{\sqrt{2}} \sin (\beta l) \\
j \sqrt{2} \sin (\beta l) & \cos (\beta l)
\end{array}\right]\left[\begin{array}{cc}
1 \\
\frac{1}{j \tan (\beta l)}
\end{array}\right] \begin{array}{c}
0 \\
1
\end{array}\right]
$$

$\left[\begin{array}{ll}A & B \\ C & D\end{array}\right]_{\text {odd }}=\left[\begin{array}{c}{\left[\cos (\beta)+\frac{\sin (\beta)}{\sqrt{2} \tan (\mathcal{l})}\right]} \\ {\left[j \frac{1}{\sqrt{2}} \sin (\beta)\right]}\end{array}\right]$

$$
T_{o}=\frac{2}{A+B+C+D}
$$

$$
\therefore T_{o}=\frac{2}{\left[\left(2 \cos (\beta)+\frac{2 \sin (\beta)}{\sqrt{2} \tan (\beta)}\right)+j\left(\frac{\sin (\beta)}{\sqrt{2}}-\frac{\cos (\beta)}{\tan (\beta)}+\sqrt{2} \sin (\not)-\frac{1}{\tan (\beta)}\left(\frac{\sin (\beta)}{\sqrt{2} \tan (\beta)}+\cos (\beta)\right)\right)\right]}
$$

The following section contains the Matlab program for the Branchline coupler. 


\section{Matlab Program \#1: Branchline Coupler}

clear all

$\mathrm{f}=30 \mathrm{e} 9$

$\mathrm{c}=3 \mathrm{e} 8$

$\operatorname{lam}=\mathrm{c} / \mathrm{f}$

$1=1 \mathrm{am} / 4$

These are the declarations for the variables used.

$\mathrm{i}=0$;

This Loop generates a matrix of data points for the amplitude and phase response

for f_span $=20 \mathrm{e} 9: 1 \mathrm{e} 9: 40 \mathrm{e} 9$

$\mathrm{i}=\mathrm{i}+1$;

Beta $=2 * \mathrm{pi}^{*} \mathrm{f} \_\mathrm{span} / \mathrm{c}$;

Beta is the propagation constant

Theta 1 is the expression for the phase at output port 2

thetal(i) $=180 / \mathrm{pi}^{*} \operatorname{atan}\left(\left(\sin \left(\right.\right.\right.$ Beta $\left.^{*} 1\right) / \operatorname{sqrt}(2)+\tan \left(\right.$ Beta $\left.^{*} 1 / 2\right) * \cos \left(\right.$ Beta $\left.^{*} 1\right)+$ $\operatorname{sqrt}(2)^{*} \sin ($ Beta*l) $\ldots$

- $\tan \left(\right.$ Beta $\left.^{*} 1 / 2\right) *\left(\tan \left(\right.\right.$ Beta $\left.^{*} 1 / 2\right) * \sin \left(\right.$ Beta $\left.^{*} 1\right) / \operatorname{sqrt}(2)-\cos \left(\right.$ Beta $\left.\left.^{*} 1\right)\right) \ldots$

$+\left(\sin \left(\right.\right.$ Beta $\left.^{*} 1\right) / \operatorname{sqrt}(2)-\cos \left(\right.$ Beta $\left.^{*} 1\right) / \tan \left(\right.$ Beta $\left.^{*} 1 / 2\right)+\operatorname{sqrt}(2) * \sin \left(\right.$ Beta $\left.^{*} 1\right) \ldots$

$-1 / \tan \left(\right.$ Beta $\left.^{*} 1 / 2\right) *\left(\sin \left(\right.\right.$ Beta $\left.^{*} 1\right) / \tan \left(\right.$ Beta $\left.^{*} 1 / 2\right) / \operatorname{sqrt}(2) \ldots$

$+\cos \left(\right.$ Beta $\left.\left.\left.\left.^{*} 1\right)\right)\right)\right) /\left(2^{*} \cos \left(\right.\right.$ Beta $\left.^{*} 1\right)-2 * \sin \left(\text { Beta }^{*} 1\right)^{*} \tan \left(\right.$ Beta $\left.^{*} 1 / 2\right) / \operatorname{sqrt}(2) \ldots$

$+\left(2 * \cos \left(\right.\right.$ Beta $\left.^{*} 1\right)+2 * \sin \left(\right.$ Beta*1)/tan $\left(\right.$ Beta $\left.\left.\left.\left.^{*} 1 / 2\right) / \operatorname{sqrt}(2)\right)\right)\right)$;

Theta2 is the expression for the phase at output port 3

theta $2(\mathrm{i})=180 / \mathrm{pi}^{*} \operatorname{atan}\left(\left(-\left(\sin \left(\right.\right.\right.\right.$ Beta $\left.^{*}\right) / \operatorname{sqrt}(2)+\tan \left(\right.$ Beta $\left.^{*} 1 / 2\right) * \cos \left(\right.$ Beta $\left.^{*} 1\right)+$ $\operatorname{sqrt}(2) * \sin \left(\right.$ Beta $\left.^{*} 1\right) \ldots$

- $\tan \left(\right.$ Beta $\left.^{*} 1 / 2\right) *\left(\tan \left(\right.\right.$ Beta $\left.^{*} 1 / 2\right) * \sin \left(\right.$ Beta $\left.^{*} 1\right) / \operatorname{sqrt}(2)-\cos \left(\right.$ Beta $\left.\left.\left.^{*} 1\right)\right)\right) \ldots$

$+\left(\sin \left(\right.\right.$ Beta*1) $^{*} / \operatorname{sqrt}(2)-\cos \left(\right.$ Beta*1 $^{*} / \tan \left(\right.$ Beta $\left.^{*} 1 / 2\right)+\operatorname{sqrt}(2) * \sin \left(\right.$ Beta $\left.^{*} 1\right) \ldots$

$-1 / \tan \left(\right.$ Beta $\left.^{*} 1 / 2\right) *\left(\sin \left(\right.\right.$ Beta $\left.^{*} 1\right) / \tan ($ Beta*1/2)/sqrt $(2) \ldots$

$+\cos \left(\right.$ Beta $\left.\left.\left.\left.^{*} 1\right)\right)\right)\right) /\left(-\left(2 * \cos \left(\right.\right.\right.$ Beta $\left.^{*} 1\right)-2 * \sin ($ Beta $* 1) * \tan ($ Beta*1/2)/sqrt $(2)) \ldots$

$+\left(2 * \cos \left(\right.\right.$ Beta $\left.^{*} 1\right)+2 * \sin \left(\right.$ Beta*1) $/ \tan \left(\right.$ Beta $\left.\left.\left.\left.^{*} 1 / 2\right) / \operatorname{sqrt}(2)\right)\right)\right)$;

This is the expression for the Transfer function

$\mathrm{S} 21(\mathrm{i})=\left(1 /\left(\left(2 * \cos \left(\right.\right.\right.\right.$ Beta $\left.^{*} 1\right)-2 * \sin \left(\right.$ Beta $\left.^{*} 1\right) * \tan \left(\right.$ Beta $\left.\left.^{*} 1 / 2\right) / \operatorname{sqrt}(2)\right)+$

$\mathrm{j}^{*}\left(\sin \left(\right.\right.$ Beta $\left.^{*} \mathrm{l}\right) / \mathrm{sqrt}(2) \ldots$

$+\tan \left(\right.$ Beta $\left.^{*} 1 / 2\right) * \cos \left(\right.$ Beta $\left.^{*} 1\right)+\operatorname{sqrt}(2) * \sin ($ Beta $* 1)-$

$\tan \left(\right.$ Beta $\left.^{*} 1 / 2\right) *\left(\tan \left(\right.\right.$ Beta $\left.^{*} 1 / 2\right) * \sin ($ Beta*1)/sqrt $(2)-\cos ($ Beta*1))) )) $\ldots$ 
$+\left(1 /\left(\left(2 * \cos \left(\right.\right.\right.\right.$ Beta $\left.^{*} 1\right)+2 * \sin \left(\right.$ Beta $\left.^{* 1}\right) / \tan \left(\right.$ Beta $\left.\left.^{*} 1 / 2\right) / \operatorname{sqrt}(2)\right)+\mathrm{j}^{*}(\sin ($ Beta*1)$/ \operatorname{sqrt}(2)-$ $\cos \left(\right.$ Beta $\left.^{*} 1\right) / \tan \left(\right.$ Beta $\left.^{*} 1 / 2\right) \ldots$

$+\operatorname{sqrt}(2) * \sin \left(\right.$ Beta $\left.^{*} 1\right)-1 / \tan \left(\right.$ Beta $\left.^{*} 1 / 2\right) *\left(\sin \left(\right.\right.$ Beta $\left.^{*} 1\right) / \tan \left(\right.$ Beta $\left.^{*} 1 / 2\right) / \operatorname{sqrt}(2)+$

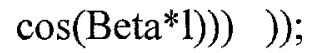

$$
\text { freq(i) =f_span; }
$$

end

figure

plot(freq,abs(theta2-theta1));

figure

$\operatorname{plot}($ freq, $20 * \log 10(\operatorname{abs}(\mathrm{S} 21)))$;

Finally, the phase response is defined as the difference in phase between port 2 and port 3 , and is plotted. The amplitude response is also plotted.

End of Branchline Coupler Program

\section{A.2 Hybrid-Ring Coupler:}

The Even and Odd mode analysis of the hybrid-ring coupler is similar to that for the branchline coupler $[10, \mathrm{p} .405]$. Based on the analysis, one finds the relations for the transfer functions for ports $2 \& 3$ to be:

$$
\begin{aligned}
& \text { Port_2 } \rightarrow S_{24}=\frac{1}{2} T_{e}+\frac{1}{2} T_{o} \\
& \text { Port_3 } \rightarrow S_{34}=\frac{1}{2} \Gamma_{e}-\frac{1}{2} \Gamma_{o}
\end{aligned}
$$

$$
\begin{aligned}
\text { Where } T_{e}=\left.\frac{2}{A+B+C+D}\right|_{e} & \Gamma e=\left.\frac{A+B-C-D}{A+B+C+D}\right|_{e} \\
T_{o}=\left.\frac{2}{A+B+C+D}\right|_{o} & \Gamma_{o}=\left.\frac{A+B-C-D}{A+B+C+D}\right|_{o}
\end{aligned}
$$


The following expressions detail how to calculate the $\mathrm{ABCD}$ matrices for the even and odd excitation of the hybrid-ring coupler and ultimately how to extract $T_{e}, T_{0}, \Gamma_{e}$, and $\Gamma_{0}$.

\section{Even-mode:}

$$
\begin{aligned}
& {\left[\begin{array}{ll}
A & B \\
C & D
\end{array}\right]_{\text {even }}=\left[\begin{array}{cc}
1 & 0 \\
j \frac{\tan \left(\beta l_{1}\right)}{\sqrt{2}} & 1
\end{array}\right]\left[\begin{array}{cc}
\cos \left(\beta l_{2}\right) & j Z_{0} \sin \left(\beta l_{2}\right) \\
j Y_{0} \sin \left(\beta l_{2}\right) & \cos \left(\beta l_{2}\right)
\end{array}\right]\left[\begin{array}{cc}
1 & 0 \\
j \frac{\tan \left(B l_{3}\right)}{\sqrt{2}} & 1
\end{array}\right]} \\
& =\left[\begin{array}{cc}
1 & 0 \\
j \frac{\tan \left(B l_{1}\right)}{\sqrt{2}} & 1
\end{array}\right]\left[\begin{array}{cc}
\cos \left(\beta l_{2}\right) & j \sqrt{2} \sin \left(\beta l_{2}\right) \\
j \frac{1}{\sqrt{2}} \sin \left(\beta l_{2}\right) & \cos \left(\beta l_{2}\right)
\end{array}\right]\left[\begin{array}{cc}
1 & 0 \\
j \frac{\tan \left(B l_{3}\right)}{\sqrt{2}} & 1
\end{array}\right]
\end{aligned}
$$

$\left[\begin{array}{ll}A & B \\ C & D\end{array}\right]_{e}=\left[\begin{array}{cc}{\left[\cos \left(A_{2}\right)-\sin \left(A_{2}\right) \tan \left(A_{3}\right)\right.} & {\left[j \sqrt{2} \sin \left(A_{2}\right)\right.}\end{array}\right]$

$$
T_{e}=\frac{2}{A+B+C+D}
$$

$$
\begin{aligned}
& \therefore T_{e}=\frac{2}{\left[\begin{array}{l}
\left(2 \cos \left(A_{2}\right)-\sin \left(\theta_{2}\right) \tan \left(\theta_{3}\right)-\sin \left(A_{2}\right) \tan \left(A_{1}\right)\right) \\
+j\left(\sqrt{2} \sin \left(A_{2}\right)+\frac{\tan \left(A_{1}\right) \cos \left(\theta_{2}\right)}{\sqrt{2}}+\frac{\sin \left(A_{2}\right)}{\sqrt{2}}-\frac{\tan \left(\ell_{3}\right)}{\sqrt{2}}\left(\tan \left(A_{1}\right) \sin \left(A_{2}\right)-\cos \left(A_{2}\right)\right)\right)
\end{array}\right]} \\
& \Gamma_{e}=\frac{A+B-C-D}{A+B+C+D}
\end{aligned}
$$




$$
\therefore \Gamma_{e}=\frac{\left[\begin{array}{l}
\left(\sin \left(\beta l_{2}\right) \tan \left(\beta l_{1}\right)-\sin \left(\beta l_{2}\right) \tan \left(\beta l_{3}\right)\right) \\
+j\left(\begin{array}{l}
\sqrt{2} \sin \left(\beta l_{2}\right)-\frac{\tan \left(\beta l_{1}\right) \cos \left(\beta l_{2}\right)}{\sqrt{2}}-\frac{\sin \left(\beta l_{2}\right)}{\sqrt{2}} \\
+\frac{\tan \left(\beta l_{3}\right)}{\sqrt{2}}\left(\tan \left(\beta l_{1}\right) \sin \left(\beta l_{2}\right)-\cos \left(\beta l_{2}\right)\right)
\end{array}\right)
\end{array}\right]}{\left[\begin{array}{l}
\left(2 \cos \left(\beta l_{2}\right)-\sin \left(\beta l_{2}\right) \tan \left(\beta l_{3}\right)-\sin \left(\beta l_{2}\right) \tan \left(\beta l_{3}\right)\right) \\
+j\left(\begin{array}{l}
\sqrt{2} \sin \left(\beta l_{2}\right)+\frac{\tan \left(\beta l_{1}\right) \cos \left(\beta l_{2}\right)}{\sqrt{2}}+\frac{\sin \left(\beta l_{2}\right)}{\sqrt{2}} \\
-\frac{\tan \left(\beta l_{3}\right)}{\sqrt{2}}\left(\tan \left(\beta l_{1}\right) \sin \left(\beta l_{2}\right)-\cos \left(\beta l_{2}\right)\right)
\end{array}\right)
\end{array}\right]}
$$

\section{Odd-mode:}

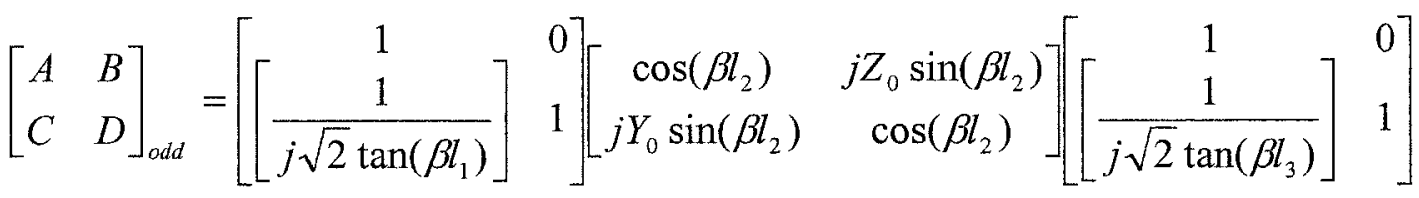

$$
\begin{aligned}
& =\left[\left[\frac{1}{j \sqrt{2} \tan \left(\beta l_{1}\right)}\right]^{0} 1\left[\begin{array}{cc}
\cos \left(\beta l_{2}\right) & j \sqrt{2} \sin \left(\beta l_{2}\right) \\
j \frac{\sin \left(\beta l_{2}\right)}{\sqrt{2}} & \cos \left(\beta l_{2}\right)
\end{array}\right]\left[\begin{array}{c}
1 \\
j \sqrt{2} \tan \left(\beta l_{3}\right)
\end{array}\right] \begin{array}{l}
0 \\
1
\end{array}\right] \\
& {\left[\begin{array}{ll}
A & B \\
C & D
\end{array}\right]_{\text {odd }}=\left[\begin{array}{cc}
{\left[\cos \left(\beta_{2}\right)+\frac{\sin \left(\beta_{2}\right)}{\tan \left(\beta_{3}\right)}\right]} & {\left[j \sqrt{2} \sin \left(\beta_{2}\right)\right]} \\
{\left[-j \frac{\cos \left(\beta_{2}\right)}{\sqrt{2} \tan \left(\beta_{1}\right)}+j \frac{\sin \left(\beta_{2}\right)}{\sqrt{2}}-\frac{j}{j \sqrt{2} \tan \left(\beta_{3}\right)}\left(\frac{\sin \left(\beta_{2}\right)}{\tan \left(\beta_{1}\right)}+\cos \left(\beta_{2}\right)\right)\right]} & {\left[\frac{\sin \left(\beta_{2}\right)}{\tan \left(\beta_{1}\right)}+\cos \left(\beta l_{2}\right)\right]}
\end{array}\right]} \\
& T_{o}=\frac{2}{A+B+C+D}
\end{aligned}
$$




$$
\begin{aligned}
& \therefore T_{o}=\frac{2}{\left[\begin{array}{l}
\left(2 \cos \left(\beta_{2}\right)+\frac{\sin \left(\beta_{2}\right)}{\tan \left(\beta_{3}\right)}+\frac{\sin \left(\beta_{2}\right)}{\tan \left(\beta_{1}\right)}\right) \\
+j\left(\sqrt{2} \sin \left(\beta_{2}\right)-\frac{\cos \left(\beta_{2}\right)}{\sqrt{2} \tan \left(\beta_{1}\right)}+\frac{\sin \left(\beta_{2}\right)}{\sqrt{2}}-\frac{1}{\sqrt{2} \tan \left(\beta_{3}\right)}\left(\frac{\sin \left(\beta_{2}\right)}{\tan \left(\beta_{1}\right)}+\cos \left(\beta_{2}\right)\right)\right]
\end{array}\right]} \\
& \Gamma_{o}=\frac{A+B-C-D}{A+B+C+D} \\
& \therefore \Gamma_{o}=\frac{\left[\begin{array}{l}
\left(\frac{\sin \left(\beta l_{2}\right)}{\tan \left(\beta l_{3}\right)}-\frac{\sin \left(\beta l_{2}\right)}{\tan \left(\beta l_{1}\right)}\right) \\
+j\left(\sqrt{2} \sin \left(\beta l_{2}\right)+\frac{\cos \left(\beta l_{2}\right)}{\sqrt{2} \tan \left(\beta l_{1}\right)}-\frac{\sin \left(\beta l_{2}\right)}{\sqrt{2}}+\frac{1}{\sqrt{2} \tan \left(\beta l_{3}\right)}\left(\frac{\sin \left(\beta l_{2}\right)}{\tan \left(\beta l_{1}\right)}+\cos \left(\beta l_{2}\right)\right)\right)
\end{array}\right]}{\left[\begin{array}{l}
\left(2 \cos \left(\beta l_{2}\right)+\frac{\sin \left(\beta l_{2}\right)}{\tan \left(\beta l_{3}\right)}+\frac{\sin \left(\beta l_{2}\right)}{\tan \left(\beta l_{1}\right)}\right) \\
+j\left(\sqrt{2} \sin \left(\beta l_{2}\right)-\frac{\cos \left(\beta l_{2}\right)}{\sqrt{2} \tan \left(\beta l_{1}\right)}+\frac{\sin \left(\beta l_{2}\right)}{\sqrt{2}}-\frac{1}{\sqrt{2} \tan \left(\beta l_{3}\right)}\left(\frac{\sin \left(\beta l_{2}\right)}{\tan \left(\beta l_{1}\right)}+\cos \left(\beta l_{2}\right)\right)\right)
\end{array}\right]}
\end{aligned}
$$

The following section contains the Matlab program for the Hybrid-Ring coupler.

\section{Matlab Program \#2: Hybrid-Ring Coupler}

clear all

$\mathrm{f}=30 \mathrm{e} 9$

$\mathrm{c}=3 \mathrm{e} 8$

$\operatorname{lam}=\mathrm{c} / \mathrm{f}$

$\mathrm{l}=\operatorname{lam} / 4$

$13=1 / 2$

These are the declarations for the variables used. 


$$
\begin{aligned}
& 12=1 \\
& 11=3 / 2 * 1
\end{aligned}
$$

This Loop generates a matrix of data points for the amplitude and phase response

for f_span = 20e9:1e9:40e9

$$
\begin{aligned}
& \mathrm{i}=\mathrm{i}+1 \\
& \mathrm{~B}=2 * \mathrm{pi}^{*} \text { __span/c; }
\end{aligned}
$$

This is the expression for reflection coefficient for even excitation

$$
\begin{aligned}
& \text { gamae }=\left(\sin \left(B^{*} 12\right) * \tan \left(B^{*} 11\right)-\sin \left(B^{*} 12\right)^{*} \tan \left(B^{*} 13\right)+j^{*}\left(\operatorname{sqrt}(2)^{*} \sin \left(B^{*} 12\right)-\right.\right. \\
& \tan \left(\mathrm{B}^{*} 11\right)^{*} \cos \left(\mathrm{B}^{*} 12\right) / \mathrm{sqrt}(2)-\sin \left(\mathrm{B}^{*} 12\right) / \operatorname{sqrt}(2) \ldots \\
& \left.\left.+\tan \left(\mathrm{B}^{*} 13\right) / \operatorname{sqrt}(2)^{*}\left(\tan \left(\mathrm{B}^{*} 11\right)^{*} \sin \left(\mathrm{B}^{*} 12\right)-\cos \left(\mathrm{B}^{*} 12\right)\right) \quad\right)\right) \\
& \left(2^{*} \cos \left(\mathrm{B}^{*} 12\right)-\sin \left(\mathrm{B}^{*} 12\right) * \tan \left(\mathrm{B}^{*} 13\right)-\sin \left(\mathrm{B}^{*} 12\right)^{*} \tan \left(\mathrm{B}^{*} 11\right) \ldots\right. \\
& +\mathrm{j}^{*}\left(\operatorname{sqrt}(2)^{*} \sin \left(\mathrm{B}^{*} 12\right)+\tan \left(\mathrm{B}^{*} 11\right)^{*} \cos \left(\mathrm{B}^{*} 12\right) / \operatorname{sqrt}(2)+\sin \left(\mathrm{B}^{*} 12\right) / \operatorname{sqrt}(2)-\right. \\
& \left.\tan \left(\mathrm{B}^{*} 13\right) / \operatorname{sqrt}(2) *\left(\tan \left(\mathrm{B}^{*} 11\right) * \sin \left(\mathrm{B}^{*} 12\right)-\cos \left(\mathrm{B}^{*} 12\right)\right)\right) \text { ); }
\end{aligned}
$$

This the expression for reflection coefficient for odd excitation

$$
\begin{aligned}
& \text { gamao }=\left(\sin \left(\mathrm{B}^{*} 12\right) / \tan \left(\mathrm{B}^{*} 13\right)-\sin \left(\mathrm{B}^{*} 12\right) / \tan \left(\mathrm{B}^{*} 11\right)+\mathrm{j}^{*}\left(\operatorname{sqrt}(2)^{*} \sin \left(\mathrm{B}^{*} 12\right)+\right.\right. \\
& \cos \left(\mathrm{B}^{*} 12\right) / \tan \left(\mathrm{B}^{*} 11\right) / \operatorname{sqrt}(2)-\sin \left(\mathrm{B}^{*} 12\right) / \operatorname{sqrt}(2) \ldots \\
& \left.\left.+1 / \operatorname{sqrt}(2) / \tan \left(\mathrm{B}^{*} 13\right)^{*}\left(\sin \left(\mathrm{B}^{*} 12\right) / \tan \left(\mathrm{B}^{*} 11\right)+\cos \left(\mathrm{B}^{*} 12\right)\right) \quad\right)\right) \\
& \left(2 * \cos \left(\mathrm{B}^{*} 12\right)+\sin \left(\mathrm{B}^{*} 12\right) / \tan \left(\mathrm{B}^{*} 13\right)+\sin \left(\mathrm{B}^{*} 12\right) / \tan \left(\mathrm{B}^{*} 11\right) \ldots\right. \\
& +\mathrm{j}^{*}\left(\operatorname{sqrt}(2)^{*} \sin \left(\mathrm{B}^{*} 12\right)-\cos \left(\mathrm{B}^{*} 12\right) / \tan \left(\mathrm{B}^{*} 11\right) / \operatorname{sqrt}(2)+\sin \left(\mathrm{B}^{*} 12\right) / \operatorname{sqrt}(2)-\right. \\
& \left.1 / \operatorname{sqrt}(2) / \tan \left(\mathrm{B}^{*} 13\right) *\left(\sin (\mathrm{B} * 12) / \tan \left(\mathrm{B}^{*} 11\right)+\cos \left(\mathrm{B}^{*} 12\right)\right)\right) \text { ); }
\end{aligned}
$$

This is the expression for S34, the transfer function to Port 3

$\mathrm{B} 2(\mathrm{i})=1 / 2 *$ gamae $-1 / 2 *$ gamao;

This is the expression for transmission coefficient for even excitation

$$
\begin{aligned}
\mathrm{Te}=2 / & \left(2^{*} \cos \left(\mathrm{B}^{*} 12\right)-\sin \left(\mathrm{B}^{*} 12\right)^{*} \tan \left(\mathrm{B}^{*} 13\right)-\sin \left(\mathrm{B}^{*} 12\right)^{*} \tan \left(\mathrm{B}^{*} 11\right)+\right. \\
& \mathrm{j}^{*}\left(\operatorname{sqrt}(2)^{*} \sin \left(\mathrm{B}^{*} 12\right)+\tan \left(\mathrm{B}^{*} 11\right) * \cos \left(\mathrm{B}^{* 12}\right) / \operatorname{sqrt}(2) \ldots\right. \\
& \left.\left.+\sin \left(\mathrm{B}^{* 12}\right) / \operatorname{sqrt}(2)-\tan \left(\mathrm{B}^{*} 13\right) / \operatorname{sqrt}(2)^{*}\left(\tan \left(\mathrm{B}^{*} 11\right)^{*} \sin \left(\mathrm{B}^{*} 12\right)-\cos \left(\mathrm{B}^{*} 12\right)\right)\right)\right)
\end{aligned}
$$

This is the expression for transmission coefficient for odd excitation 


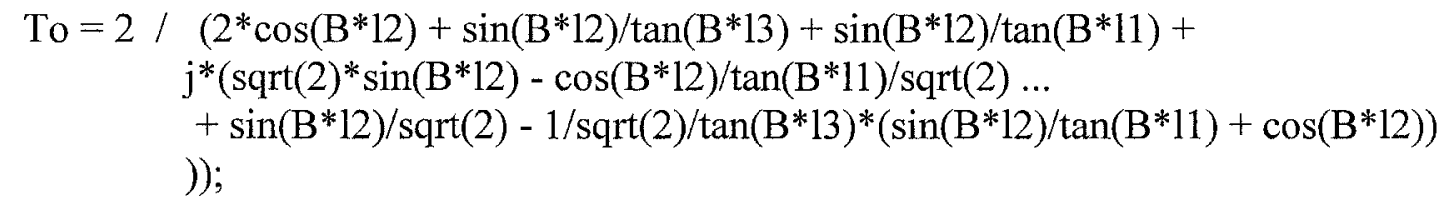

This is the expression for $\mathrm{S} 24$, the transfer function to Port 2

$$
\begin{aligned}
& \mathrm{B} 3(\mathrm{i})=1 / 2 * \mathrm{Te}+1 / 2 * \mathrm{To} \\
& \text { freq(i) =f_span; }
\end{aligned}
$$

end

thetal $=180 / \mathrm{pi}^{*}$ angle(B2);

theta2 = 180/pi*angle(B3);

figure

plot(freq,abs(theta1-theta2));

figure

plot(freq, $20 * \log 10(\operatorname{abs}(\mathrm{B} 3)))$

Finally, the phase response is defined as the difference in phase between port 2 and port 3 , and is plotted. The amplitude response is also plotted.

End of Hybrid-Ring Coupler Program

\section{A.3 Coupled-Line Coupler:}

The transfer functions for port 2 and 3 of the coupled-line coupler are taken directly from $[10, p .391]$. These are given in terms of voltages as follows:

$\frac{V_{2}}{V}=\frac{\sqrt{1-C^{2}}}{\sqrt{1-C^{2}} \cos \theta+j \sin \theta}$

And

$$
\frac{V_{3}}{V}=\frac{j C \tan \theta}{\sqrt{1-C^{2}+j \tan \theta}}
$$


Where $C=\frac{Z_{0 e}-Z_{0 o}}{Z_{0 e}+Z_{00}}$

The following section contains the Matlab program for the Coupled-Line coupler.

\section{Matlab Program \#3: Coupled-Line Coupler}

clear all

$\mathrm{f}=30 \mathrm{e} 9$

$\mathrm{c}=3 \mathrm{e} 8$

$\operatorname{lam}=\mathrm{c} / \mathrm{f}$;

$1=\operatorname{lam} / 4$

$\mathrm{Ze}=100$

$Z o=25$

These are the declarations for the variables used.

$\mathrm{C}=(\mathrm{Ze}-\mathrm{Zo}) /(\mathrm{Ze}+\mathrm{Zo})$

$\mathrm{i}=0$;

This Loop generates a matrix of data points for the amplitude and phase response

for $f_{-}$span $=20 \mathrm{e} 9: 1 \mathrm{e} 9: 40 \mathrm{e} 9$

$\mathrm{i}=\mathrm{i}+1$;

$\mathrm{B}=2 * \mathrm{pi}^{*} \mathrm{f}_{-} \mathrm{span} / \mathrm{c}$;

This is the expression for the transfer function for Port 3

$V 3(i)=j^{*}\left(C^{*} \tan \left(B^{*} 1\right)\right) /\left(\operatorname{sqrt}\left(1-C^{\wedge} 2\right)+j^{*} \tan \left(B^{*} l\right)\right)$;

This is the expression for the transfer function for Port 2

$\mathrm{V} 2(\mathrm{i})=\operatorname{sqrt}\left(1-\mathrm{C}^{\wedge} 2\right) /\left(\operatorname{sqrt}\left(1-\mathrm{C}^{\wedge} 2\right)^{*} \cos \left(\mathrm{B}^{*} \mathrm{l}\right)+\mathrm{j}^{*} \sin \left(\mathrm{B}^{*} 1\right)\right)$;

freq(i) $=f \_s p a n ;$ 
end

theta $1=180 / \mathrm{pi}^{*}$ angle(V2);

theta $2=180 /$ pi*angle(V3);

$\mathrm{mx}=\max (\mathrm{V} 3)$

$\mathrm{V} 3=\mathrm{V} 3 / \mathrm{mx}$

figure

plot(freq,abs(theta2-theta1));

figure

$\operatorname{plot}\left(\right.$ freq, $\left.(\operatorname{abs}(\mathrm{V} 2)) .^{\wedge} 2\right)$;

figure

plot(freq, 10* $\log ((\operatorname{abs}(\mathrm{V} 3)) . \wedge 2))$;

Finally, the phase response is defined as the difference in phase between port 2 and port 3 , and is plotted. The amplitude response is also plotted.

End of Coupled-Line Coupler Program 